\title{
Applications of Almgren-Pitts Min-max theory
}

\author{
Fernando C. Marques and André Neves
}

\begin{abstract}
We survey recent applications of Almgren-Pitts Min-max theory found by the authors. Namely, the proof of the Willmore conjecture, the proof of the Freedman-He-Wang conjecture on the Möbius energy of links (jointly with Ian Agol), and the proof that manifolds with positive Ricci curvature metrics admit an infinite number of minimal hypersurfaces.
\end{abstract}

\section{Contents}

1. Introduction

2. Almgren-Pitts Min-max theory I 11

3. Criterion for a 5-cycle in $\mathcal{Z}_{2}\left(S^{3}\right)$ to be nontrivial. 20

4. Canonical family of surfaces 24

5. $2 \pi^{2}$-Theorem 34

6. Application: The Willmore Conjecture 40

7. Canonical family of links 40

8. Application: Freedman-He-Wang Conjecture 47

9. Gromov-Guth families 48

10. Application: Existence of infinitely many minimal hypersurfaces 51

11. Almgren-Pitts Min-max theory II 55

12. Proof of Min-Max Theorems of Section 2

References $\quad 70$

\section{Introduction}

The relation between critical points of a functional and the topology of the underlying space, due to Morse, is one of the most insightful ideas in Geometry.

In the Riemannian context, a natural pair to consider is the space of all hypersurfaces in given manifold and the functional which assigns to each hypersurface its volume. In this case, the critical points of the functional are 
called stationary or minimal hypersurfaces. The general philosophy consists in finding some nontrivial topology in the space of all hypersurfaces and then use that to better understand the geometric properties of the ambient manifold.

Almgren and Pitts undertook the study of Morse Theory in the space of all hypersurfaces in the late $70^{\prime}$ s and developed what it is now called Almgren-Pitts Min-max Theory. The aim of this notes is to explain how we recently used Almgren-Pitts theory to solve some open problems in geometry.

We now describe the key geometric principles involved in a somewhat informal way. The definitions and detailed work can be seen from Section 2 to Section 12.

1.1. Almgren-Pitts Min-max Theory: An overview. For the sake of simplicity, the reader can think of $\mathcal{Z}_{n}(M)$ as being the space of all orientable compact hypersurfaces with possible multiplicities in a compact Riemannian $(n+1)$-manifold $(M, g)$. If we allow for non-orientable hypersurfaces as well, the space is denoted by $\mathcal{Z}_{n}\left(M ; \mathbb{Z}_{2}\right)$. These spaces come equipped with a natural topology (flat topology) and with a natural functional, the mass functional $\mathbf{M}$, which associated to every element in $\mathcal{Z}_{n}(M)\left(\right.$ or $\mathcal{Z}_{n}\left(M ; \mathbb{Z}_{2}\right)$ ) its volume.

As it was previously mentioned, smooth minimal hypersurfaces are critical points for the mass functional and their index is the number of negative eigenvalues for the second variation of the mass functional. It corresponds to the maximum number of linearly independent directions in which we can strictly decrease the volume of a minimal hypersurface.

Let $G$ be either the group of integers or $\mathbb{Z}_{2}$. The guiding principle of Almgren-Pitts Min-max Theory, just like Morse Theory, is that a homotopically non-trivial $k$-dimensional cycle in $\mathcal{Z}_{n}(M ; G)$ should produce a minimal hypersurface with index at most $k$.

Being more precise, suppose $X$ is a topological space and $\Phi: X \rightarrow$ $\mathcal{Z}_{n}(M ; G)$ a continuous function. Consider

$$
[\Phi]=\left\{\Psi: X \rightarrow \mathcal{Z}_{n}(M ; G): \Psi \text { homotopic to } \Phi\right\}
$$

or, in case of relative homotopies,

$$
[\Phi]=\left\{\Psi: X \rightarrow \mathcal{Z}_{n}(M ; G): \Psi \text { homotopic to } \Phi \text { relative to } \partial X\right\}
$$

To each homotopy class $[\Phi]$ we associate the number

$$
\mathbf{L}([\Phi])=\inf _{\Psi \in[\Phi]} \sup _{x \in X} \mathbf{M}(\Psi(x)) .
$$

The Almgren-Pitts Min-max Theorem [37] (see also [40]) can be stated as 
Min-max Theorem. Assume $2 \leq n \leq 6$.

If $\mathbf{L}([\Phi])>0$ or, in the relative case, $\mathbf{L}([\Phi])>\sup _{x \in \partial X} \mathbf{M}(\Phi(x))$, there is a compact embedded minimal hypersurface $\Sigma$ (with possible multiplicities) such that

$$
\mathbf{L}([\Phi])=\mathbf{M}(\Sigma) .
$$

The Min-max Theorem allows for $\Sigma$ to be a union of disjoint hypersurfaces, each with some multiplicity. More precisely

$$
\Sigma=n_{1} \Sigma_{1}+\ldots+n_{k} \Sigma_{k},
$$

where $n_{i} \in \mathbb{N}, i=1, \ldots, k$, and $\left\{\Sigma_{1}, \ldots, \Sigma_{k}\right\}$ are smooth embedded minimal surfaces with disjoint supports. Naturally, if the space of parameters $X$ is a $k$-dimensional space, we expect the index of $\Sigma$, i.e., the number of linearly independent deformations that decrease the area of $\Sigma$, to be less or equal than $k$. Unfortunately, this fact has not been proven.

If $M$ has dimension greater than or equal to 8, then Min-max Theorem is still valid but, according to the regularity theory developed by Schoen and Simon [40], $\Sigma$ is only guaranteed to be smooth and embedded outside a set of Hausdorff Hausdorff codimension 7.

The condition $\mathbf{L}([\Phi])>0\left(\right.$ or $\mathbf{L}([\Phi])>\sup _{x \in \partial X} \mathbf{M}(\Phi(x))$ in the relative case) implies that $[\Phi]$ is capturing some nontrivial topology of $\mathcal{Z}_{n}(M ; G)$. The basic structure behind the work described in this notes consists in finding examples of homotopy classes satisfying this condition and then use (1) to deduce some geometric consequences.

The next example illustrates well this methodology. Given $f: M \rightarrow[0,1]$ a Morse function, consider the continuous map

$$
\Phi:[0,1] \rightarrow \mathcal{Z}_{n}(M), \quad \Phi(t)=\partial\{x \in M: f(x)<t\} .
$$

We have $\Phi(0)=\Phi(1)=0$ and Almgren showed in $[\mathbf{2}]$ that $L([\Phi])>0$, i.e., $[\Phi]$ is a nontrivial element in $\pi_{1}\left(\mathcal{Z}_{n}(M),\{0\}\right)$. Using (1), one obtains the existence of a smooth embedded minimal hypersurface in $(M, g)$. This application was one of the motivations for Almgren and Pitts to develop their Min-max Theory. Birkhoff [7] in 1917 used a similar approach to show that every metric on a 2 -sphere admits a closed geodesic.

1.2. Criterion for a 5-cycle in $\mathcal{Z}_{2}\left(S^{3}\right)$ to be nontrivial. Let $I^{k}$ denote a $k$-dimensional cube. $B_{r}(p)$ denotes the geodesic ball in $S^{3}$ of radius $r$ centered at $p$.

We present, in general terms, a criterion to ensure that a map $\Phi: I^{5} \rightarrow$ $\mathcal{Z}_{2}\left(S^{3}\right)$ determines a nontrivial homotopy class.

Let $\mathcal{G}_{o}$ be the set of all oriented geodesic spheres in $\mathcal{Z}_{2}\left(S^{3}\right)$. Each nonzero element in $\mathcal{G}_{o}$ is determined by its center and radius. Thus this space is homeomorphic to $S^{3} \times[-\pi, \pi]$ with an equivalence relation that identifies $S^{3} \times\{-\pi\}$ and $S^{3} \times\{\pi\}$ all with the same point.

The maps $\Phi$ we consider have the property that

$$
\Phi\left(I^{4} \times\{1\}\right)=\Phi\left(I^{4} \times\{0\}\right)=\{0\} \text { and } \Phi\left(\partial I^{5}\right) \subset \mathcal{G}_{o} .
$$


Hence $\Phi\left(I^{5}\right)$ can be thought of as a 5-cycle in $\mathcal{Z}_{2}\left(S^{3}\right)$ whose boundary lies in $\mathcal{G}_{o}$ and thus $[\Phi]$ can be seen as an element of $\pi_{5}\left(\mathcal{Z}_{2}\left(S^{3}\right), \mathcal{G}_{o}\right)$. The next theorem gives a condition under which $[\Phi] \neq 0$ in $\pi_{5}\left(\mathcal{Z}_{2}\left(S^{3}\right), \mathcal{G}_{o}\right)$.

1.3. Theorem. Let $\Phi: I^{5} \rightarrow \mathcal{Z}_{2}\left(S^{3}\right)$ be a continuous map such that:

(1) $\Phi(x, 0)=\Phi(x, 1)=0$ for all $x \in I^{4}$;

(2) for all $x \in \partial I^{4}$ we can find $Q(x) \in S^{3}$ such that

$$
\Phi(x, t)=\partial B_{\pi t}(Q(x)), \quad 0 \leq t \leq 1
$$

(3) the map $Q: \partial I^{4} \rightarrow S^{3}$ has $\operatorname{deg}(Q) \neq 0$.

Then

$$
\mathbf{L}([\Phi])>4 \pi=\sup _{x \in \partial I^{5}} \mathbf{M}(\Phi(x))
$$

1.4. Minimal surfaces with low index. The simplest minimal surfaces in $S^{3}$ are great spheres and the Clifford torus $S^{1}\left(\frac{1}{\sqrt{2}}\right) \times S^{1}\left(\frac{1}{\sqrt{2}}\right)$. Lawson [26] found infinitely many distinct minimal surfaces in $S^{3}$ but these two are the only ones that have an explicit description.

Great spheres in $S^{3}$ have index one because if we move a great sphere in the direction of its normal vector with constant speed the area decreases and any deformation of the great sphere that fixes the enclosed volume never decreases the area. The Clifford torus has index five and the space of linearly independent deformations that decrease the area is spanned by constant speed normal deformations and the four dimensional space consisting of conformal deformations modulo isometries (to be seen later).

Urbano in 1990 classified minimal surface of $S^{3}$ with low index and he showed

Urbano's Theorem. Assume $\Sigma$ is a closed embedded minimal surface in $S^{3}$ having index $(\Sigma) \leq 5$.

Then $\Sigma$ is either a great sphere (index one) or the Clifford torus (index five) up to ambient isometries.

The proof is very geometric and given in Section 5 .

Given $\Phi$ satisfying the conditions of Theorem 1.3, we have that

$$
\mathbf{L}([\Phi])>\sup _{x \in \partial I^{5}} \mathbf{M}(\Phi(x))=4 \pi
$$

and thus we can apply the Min-max Theorem to conclude the existence of a minimal surface $\Sigma$ with $\mathbf{L}([\Phi])=\mathbf{M}(\Sigma)$. It is natural to expect that $\Sigma$ has index at most five because we are dealing with a 5-parameter family of surfaces. On the other hand, we know from Urbano's Theorem that, up to rigid motions, there are only two minimal surfaces of $S^{3}$ with index at most five: the great sphere and the Clifford torus. If $\Sigma$ were the great sphere then $\mathbf{L}([\Phi])=4 \pi$ and this is impossible by Theorem 1.3. Hence $\Sigma$ has to be the Clifford torus (with area $2 \pi^{2}$ ) and so we obtain 
$2 \pi^{2}$-Theorem. Given $\Phi$ satisfying the conditions of Theorem 1.3 we have

$$
\sup _{x \in I^{5}} \mathbf{M}(\Phi(x)) \geq 2 \pi^{2} .
$$

Next, we outline applications of this theorem.

1.5. Application: Willmore Conjecture. The Willmore energy of a closed surface $\Sigma$ immersed in $\mathbb{R}^{3}$ is

$$
W(\Sigma)=\int_{\Sigma}\left(\frac{k_{1}+k_{2}}{2}\right)^{2} d \mu=\int_{\Sigma} H^{2} d \mu
$$

where $k_{1}, k_{2}$ are the principal curvatures of $\Sigma, H=\frac{k_{1}+k_{2}}{2}$ the mean curvature, and $d \mu$ the area form of $\Sigma$.

The Willmore energy is obviously invariant under isometries of $\mathbb{R}^{3}$ and, less obvious, is the fact already known to Blaschke [8] and Thomsen [43] in the 1920s that this energy is also conformally invariant, i.e., $W(F(\Sigma))=$ $W(\Sigma)$ for any conformal map $F$ of $\mathbb{R}^{3}$.

In the 1960s, Willmore proved the following result:

Theorem. Let $\Sigma$ be a smooth closed surface in $\mathbb{R}^{3}$. Then $W(\Sigma) \geq 4 \pi$, and equality holds if and only if $\Sigma$ is a round sphere.

Thus, in some sense, the Willmore energy measures the deviation of a given surface from being round. It is then natural to ask what is the smallest possible Willmore energy among all surfaces of fixed topological type. Motivated by the analysis of circular tori of revolution, Willmore made a conjecture [45] for the case of genus one: $2 \pi^{2}$.

Willmore Conjecture (1965). Every torus immersed in $\mathbb{R}^{3}$ has $W(\Sigma) \geq$

The equality is achieved by the torus of revolution whose generating circle has radius 1 and center at distance $\sqrt{2}$ from the axis of revolution:

$$
(u, v) \mapsto((\sqrt{2}+\cos u) \cos v,(\sqrt{2}+\cos u) \sin v, \sin u) \in \mathbb{R}^{3} .
$$

The Willmore conjecture has been a heavily studied problem in Differential Geometry with several contributions by many authors. For some history of the problem and a collection of the progress made around the conjecture, the reader can see $[\mathbf{3 1}]$ or the survey $[\mathbf{3 3}]$.

The conformal invariance of the Willmore energy implies that it transforms nicely under stereographic projections. Indeed, if $\pi: S^{3} \backslash\{p\} \rightarrow \mathbb{R}^{3}$ denotes a stereographic projection map, the reader can check that for every surface $\Sigma \subset S^{3} \backslash\{p\}$ and denoting $\tilde{\Sigma}=\pi(\Sigma) \subset \mathbb{R}^{3}$, we have

$$
\int_{\tilde{\Sigma}} \tilde{H}^{2} d \tilde{\mu}=\int_{\Sigma}\left(1+H^{2}\right) d \mu
$$

where $H, d \mu$ and $\tilde{H}, d \tilde{\mu}$ are the mean curvature functions and area forms of $\Sigma \subset S^{3}$ and $\tilde{\Sigma} \subset \mathbb{R}^{3}$, respectively. 
In light of this, we define the Willmore energy of a surface $\Sigma$ in $S^{3}$ to be

$$
\mathcal{W}(\Sigma)=\int_{\Sigma}\left(1+H^{2}\right) d \mu
$$

As we explain shortly, the Willmore conjecture follows from the next theorem, which we proved in $[\mathbf{3 1}]$ :

Theorem A. Let $\Sigma \subset S^{3}$ be an embedded, smooth closed surface of genus $g \geq 1$. Then

$$
\mathcal{W}(\Sigma) \geq 2 \pi^{2}
$$

and the equality holds if and only if $\Sigma$ is the Clifford torus up to conformal transformations of $S^{3}$.

A corollary of Theorem A is

Corollary. The Willmore conjecture holds.

Proof. Let $\tilde{\Sigma}$ be a genus one surface in $\mathbb{R}^{3}$. If $\tilde{\Sigma}$ is immersed it was shown by Li and Yau [27] that $W(\tilde{\Sigma}) \geq 8 \pi$ and thus we can assume that $\tilde{\Sigma}$ is embedded. Denoting by $\Sigma=\pi^{-1}(\tilde{\Sigma}) \subset S^{3}$, we have from Theorem $A$ that $W(\tilde{\Sigma})=\mathcal{W}(\Sigma) \geq 2 \pi^{2}$.

The idea to prove Theorem A is the following.

The conformal maps of $S^{3}$ (modulo isometries) can be parametrized by the unit 4-ball $B^{4}$, where to each nonzero $v \in B^{4}$ we consider the conformal dilation $F_{v}$ centered at $v /|v|$ and $-v /|v|$. Composing with the stereographic projection $\pi: S^{3} \backslash\{-v /|v|\} \rightarrow \mathbb{R}^{3}$, the map $\pi \circ F_{v} \circ \pi^{-1}$ corresponds to a dilation in space, where the dilation factor tends to infinity as $|v|$ tends to one.

Given a compact embedded surface $S \subset S^{3}$ and $-\pi \leq t \leq \pi$, we denote by $S_{t}$ the surface at distance $|t|$ from $S$, where $S_{t}$ lies in the exterior (interior) of $S$ if $t \geq 0(t \leq 0)$. Naturally, $S_{t}$ might not be a smooth embedded surface due to the existence of possible focal points but it will always be well defined in the context of Geometric Measure Theory.

We can now define the following 5-parameter family $\left\{\Sigma_{(v, t)}\right\}_{(v, t) \in B^{4} \times[\pi, \pi]}$ of surfaces in $S^{3}$ given by

$$
\Sigma_{(v, t)}=\left(F_{v}(\Sigma)\right)_{t} \in \mathcal{Z}_{2}\left(S^{3}\right) .
$$

One crucial property of this 5-parameter family, to be proven later, is the following.

1.6. Heintze-Karcher Inequality. For every $(v, t) \in B^{4} \times[\pi, \pi]$ we have

$$
\mathbf{M}\left(\Sigma_{(v, t)}\right) \leq \mathcal{W}(\Sigma)
$$

The Heintze-Karcher Inequality is more general than the one stated above. The connection with the Willmore energy was made by Ros in [39].

After a careful analysis of the behaviour of $\Sigma_{(v, t)}$ as $(v, t)$ approaches the boundary of $B^{4} \times[\pi, \pi]$, we reparametrize it and obtain a continuous 
map $\Phi: I^{5} \rightarrow \mathcal{Z}_{2}\left(S^{3}\right)$ that satisfies conditions (1) and (2) of Theorem 1.3. Moreover, $\Phi\left(I^{5}\right)$ is equal to the closure of $\left\{\Sigma_{(v, t)}\right\}_{(v, t) \in B^{4} \times[\pi, \pi]}$ in $\mathcal{Z}_{2}\left(S^{3}\right)$ and thus we obtain from the Heintze-Karcher Inequaltiy that

$$
\sup _{x \in I^{5}} \mathbf{M}(\Phi(x)) \leq \mathcal{W}(\Sigma) .
$$

Finally, and most important of all, we show that if the genus of $\Sigma$ is positive then condition (3) of Theorem 1.3 is also satisfied. Thus we obtain from the $2 \pi^{2}$-Theorem that

$$
2 \pi^{2} \leq \sup _{x \in I^{5}} \mathbf{M}(\Phi(x)) \leq \mathcal{W}(\Sigma) .
$$

This proves the inequality in Theorem A.

1.7. Application: Freedman-He-Wang Conjecture. The second application comes from the theory of links in $\mathbb{R}^{3}$. Let $\gamma_{i}: S^{1} \rightarrow \mathbb{R}^{3}, i=1,2$, be a 2 -component link, i.e., a pair of closed curves in Euclidean three-space with $\gamma_{1}\left(S^{1}\right) \cap \gamma_{2}\left(S^{1}\right)=\emptyset$.

Every 2-component link has a numerical invariant called the linking number $\operatorname{lk}\left(\gamma_{1}, \gamma_{2}\right)$. Intuitively, it measures how many times each curve winds around the other and it can be computed via the Gauss formula

$$
\operatorname{lk}\left(\gamma_{1}, \gamma_{2}\right)=\frac{1}{4 \pi} \int_{S^{1} \times S^{1}} \frac{\operatorname{det}\left(\gamma_{1}^{\prime}(s), \gamma_{2}^{\prime}(t), \gamma_{1}(s)-\gamma_{2}(t)\right)}{\left|\gamma_{1}(s)-\gamma_{2}(t)\right|^{3}} d s d t .
$$

To every 2-component link $\left(\gamma_{1}, \gamma_{2}\right)$ we can associate an energy, called the Möbius cross energy. Its definition is reminiscent of the electrostatic potential energy and is given by $([\mathbf{3 6}],[\mathbf{1 5}])$ :

$$
E\left(\gamma_{1}, \gamma_{2}\right)=\int_{S^{1} \times S^{1}} \frac{\left|\gamma_{1}^{\prime}(s)\right|\left|\gamma_{2}^{\prime}(t)\right|}{\left|\gamma_{1}(s)-\gamma_{2}(t)\right|^{2}} d s d t .
$$

Like the Willmore energy, the Möbius energy has the remarkable property of being invariant under conformal transformations of $\mathbb{R}^{3}[\mathbf{1 5}]$. Note that the definition of the energy and its conformal invariance property extend to any 2-component link in $\mathbb{R}^{n}[\mathbf{2 4}]$.

Because of Gauss formula for the linking number, we immediately get that $E\left(\gamma_{1}, \gamma_{2}\right) \geq 4 \pi\left|\operatorname{lk}\left(\gamma_{1}, \gamma_{2}\right)\right|$. It is then natural to search for optimal configurations, i.e., minimizers of the Möbius energy. Freedman, He and Wang [15] considered this question and looking at the particular case where one of the link components is a planar circle, they made the following conjecture.

Conjecture (1994). The Möbius energy should be minimized, among the class of all nontrivial 2-component links in $\mathbb{R}^{3}$, by the stereographic projection of the standard Hopf link in $S^{3}$.

The standard Hopf link $\left(\hat{\gamma}_{1}, \hat{\gamma}_{2}\right)$ in $S^{3}$ is described by

$$
\hat{\gamma}_{1}(s)=(\cos s, \sin s, 0,0) \in S^{3} \quad \text { and } \quad \hat{\gamma}_{2}(t)=(0,0, \cos t, \sin t) \in S^{3},
$$

and it is simple to check that $E\left(\hat{\gamma}_{1}, \hat{\gamma}_{2}\right)=2 \pi^{2}$.

In a joint work with Agol $[\mathbf{1}]$ we showed 
Theorem B. Let $\gamma_{i}: S^{1} \rightarrow \mathbb{R}^{3}, i=1,2$, be a 2-component link in $\mathbb{R}^{3}$ with $\left|\operatorname{lk}\left(\gamma_{1}, \gamma_{2}\right)\right|=1$. Then $E\left(\gamma_{1}, \gamma_{2}\right) \geq 2 \pi^{2}$.

Moreover, if $E\left(\gamma_{1}, \gamma_{2}\right)=2 \pi^{2}$ then there exists a conformal map $F$ : $\mathbb{R}^{4} \rightarrow \mathbb{R}^{4}$ such that $\left(F \circ \gamma_{1}, F \circ \gamma_{2}\right)$ describes the standard Hopf link up to orientation.

A previous result of He [22] said that it suffices to prove the conjecture for links $\left(\gamma_{1}, \gamma_{2}\right)$ with linking number $\operatorname{lk}\left(\gamma_{1}, \gamma_{2}\right)= \pm 1$ and so Theorem $\mathrm{B}$ implies the conjecture of Freedman, He, and Wang.

The approach to prove Theorem B is similar to the one used in Theorem A. The conformal invariance of the energy implies that it suffices to consider links $\left(\gamma_{1}, \gamma_{2}\right)$ in $S^{3}$. For each link $\left(\gamma_{1}, \gamma_{2}\right)$ in $S^{3}$ we construct a suitable family $\Phi: I^{5} \rightarrow \mathcal{Z}_{2}\left(S^{3}\right)$ that satisfies conditions (1) and (2) of Theorem 1.3. Moreover, we will also show that if $\left|\operatorname{lk}\left(\gamma_{1}, \gamma_{2}\right)\right|=1$ then condition (3) of Theorem 1.3 is will also be satisfied. Hence we can apply the $2 \pi^{2}$-Theorem and conclude that

$$
\sup _{x \in I^{5}} \mathbf{M}(\Phi(x)) \geq 2 \pi^{2}
$$

On the other hand, the map $\Phi$ is constructed so that $\mathbf{M}(\Phi(x)) \leq E\left(\gamma_{1}, \gamma_{2}\right)$ for each $x \in I^{5}$ and this implies the inequality in Theorem B.

We give a brief indication of how the map $\Phi$ is constructed.

To every pair of curves in $\mathbb{R}^{4}$ there is a natural way to construct a "torus" in $S^{3}$. More precisely, given two curves $\left(\gamma_{1}, \gamma_{2}\right)$ in $\mathbb{R}^{4}$, the Gauss map $g=G\left(\gamma_{1}, \gamma_{2}\right)$ is denoted by

$$
g: S^{1} \times S^{1} \rightarrow S^{3}, \quad g(s, t)=\frac{\gamma_{1}(s)-\gamma_{2}(t)}{\left|\gamma_{1}(s)-\gamma_{2}(t)\right|}
$$

and we consider the push forward current (see [41] for definition) $G\left(\gamma_{1}, \gamma_{2}\right)_{\#}\left(S^{1} \times\right.$ $\left.S^{1}\right)$ in $\mathcal{Z}_{2}\left(S^{3}\right)$. Furthermore, one can check that

$$
\mathbf{M}\left(G\left(\gamma_{1}, \gamma_{2}\right)_{\#}\left(S^{1} \times S^{1}\right)\right) \leq E\left(\gamma_{1}, \gamma_{2}\right)
$$

For instance, if $\left(\gamma_{1}, \gamma_{2}\right)$ is the Hopf link then $G\left(\gamma_{1}, \gamma_{2}\right)_{\#}\left(S^{1} \times S^{1}\right)$ is the Clifford torus and the inequality above becomes an equality.

Given $v \in B^{4}$, we consider the conformal map $F_{v}$ of $\mathbb{R}^{4}$ given by an inversion centered at $v$. The conformal map $F_{v}$ sends the unit 4-ball $B^{4}$ to some other ball centered at $c(v)=\frac{v}{1-|v|^{2}}$. We consider $g: B^{4} \times(0,+\infty) \rightarrow$ $\mathcal{Z}_{2}\left(S^{3}\right)$ given by

$$
g(v, \lambda)=G\left(F_{v} \circ \gamma_{1}, \lambda\left(F_{v} \circ \gamma_{2}-c(v)\right)+c(v)\right)_{\#}\left(S^{1} \times S^{1}\right)
$$

Intuitively, $g(v, \lambda)$ is the image of the Gauss map of the link obtained by applying the conformal transformation $F_{v}$ to $\left(\gamma_{1}, \gamma_{2}\right)$ and then dilating the curve $F_{v} \circ \gamma_{2}$ with respect to the center $c(v)$ by a factor of $\lambda$. Note that both curves $F_{v} \circ \gamma_{1}$ and $\lambda\left(F_{v} \circ \gamma_{2}-c(v)\right)+c(v)$ are contained in spheres centered at $c(v)$. 
A Heintze-Karcher type inequality also holds for the 5-parameter family we just described, meaning that for all $(v, \lambda) \in B^{4} \times(0,+\infty)$ we have

$$
\mathbf{M}(g(v, \lambda)) \leq E\left(\gamma_{1}, \gamma_{2}\right) .
$$

The map $\Phi$ is constructed via a reparametrization of $g$.

1.8. Gromov-Guth families. The homotopy groups of the space $\mathcal{Z}_{n}\left(M ; \mathbb{Z}_{2}\right)$ can be computed through the work of Almgren [2]. It follows that all homotopy groups vanish but the first one and $\pi_{1}\left(\mathcal{Z}_{n}\left(M ; \mathbb{Z}_{2}\right)\right)=\mathbb{Z}_{2}$. Thus $\mathcal{Z}_{n}\left(M ; \mathbb{Z}_{2}\right)$ is weakly homotopic equivalent to $\mathbb{R P}^{\infty}$ and so we should expect that $\mathcal{Z}_{n}\left(M ; \mathbb{Z}_{2}\right)$ contains, for every $p \in \mathbb{N}$, a homotopically nontrivial $p$-dimensional projective space.

With that in mind, Gromov $[\mathbf{1 7}, \mathbf{1 8}, \mathbf{1 9}]$ and Guth $[\mathbf{2 0}]$ studied $p$ sweepouts of $M$. Heuristically, given a simplicial complex $X$, a continuous $\operatorname{map} \Phi: X \rightarrow \mathcal{Z}_{n}\left(M ; \mathbb{Z}_{2}\right)$ is called a $p$-sweepout if for every set $\left\{x_{1}, \ldots, x_{p}\right\} \subset$ $M$, there is $\theta \in X$ so that $\left\{x_{1}, \ldots, x_{p}\right\} \subset \Phi(\theta)$.

Denote the set of all $p$-sweepouts of $M$ by $\mathcal{P}_{p}$.

For instance, let $f \in C^{\infty}(M)$ be a Morse function and consider the map

$$
\Phi: \mathbb{R P}^{p} \rightarrow \mathcal{Z}_{n}\left(M ; \mathbb{Z}_{2}\right),
$$

given by

$$
\Phi\left(\left[a_{0}, \ldots, a_{p}\right]\right)=\partial\left\{x \in M: a_{0}+a_{1} f(x)+\ldots+a_{p} f^{p}(x)<0\right\} .
$$

Note that the map $\Phi$ is well defined because opposite orientations on the same hypersurface determine the same element in $\mathcal{Z}_{n}\left(M ; \mathbb{Z}_{2}\right)$. It is easy to see that $\Phi$ satisfies the heuristic definition of a $p$-sweepout given above.

The $p$-width of $M$ is defined as

$$
\omega_{p}(M)=\inf _{\Phi \in \mathcal{P}_{p}} \sup \{\mathbf{M}(\Phi(x)): x \in \operatorname{dmn}(\Phi)\},
$$

where $\operatorname{dmn}(\Phi)$ is the domain of $\Phi$.

It is interesting to compare the $p$-width with the min-max definition of the $p^{t h}$-eigenvalue of $(M, g)$. Set $V=W^{1,2}(M) \backslash\{0\}$ and recall that

$$
\lambda_{p}=\inf _{(p+1)-\text { plane } P \subset V} \max \left\{\frac{\int_{M}|\nabla f|^{2} d V_{g}}{\int_{M} f^{2} d V_{g}}: f \in P\right\} .
$$

Hence one can see $\left\{\omega_{p}(M)\right\}_{p \in \mathbb{N}}$ as a nonlinear analogue of the Laplace spectrum of $M$, as proposed by Gromov [17].

Gromov and Guth's Theorem. There exists a positive constant $C=$ $C(M, g)$ so that, for every $p \in \mathbb{N}$,

$$
C^{-1} p^{\frac{1}{n+1}} \leq \omega_{p}(M) \leq C p^{\frac{1}{n+1}} .
$$

The idea to prove the lower bound is, roughly speaking, the following. Choose $p$ disjoint geodesic balls $B_{1}, \ldots, B_{p}$ with radius proportional to $p^{-\frac{1}{n+1}}$. For every $p$-sweepout $\Phi$ one can find $\theta \in \operatorname{dmn}(\Phi)$ so that $\Phi(\theta)$ divides each geodesic ball into two pieces with almost identical volumes. Hence 
the isoperimetric inequality implies $\Phi(\theta) \cap B_{i}$ has volume no smaller than $c p^{-\frac{n}{n+1}}$ for all $i=1, \ldots, p$, where $c=c(M, g)$ is a constant depending only on $(M, g)$. As a result $\Phi(\theta)$ has volume greater than or equal to $c p^{\frac{1}{n+1}}$.

The upper bound can be proven using a bend-and-cancel argument introduced by Guth $[\mathbf{2 0}]$. In the case when $M$ is a $(n+1)$-dimensional sphere $S^{n+1}$, the upper bound has the following simple explanation: If we consider the space spanned by all homogenous harmonic polynomials in $S^{n+1}$ with degree less or equal than $d \in \mathbb{N}$, we obtain a vector space of dimension $p(d)+1$, where $p(d)$ grows like $d^{n+1}$. Considering the zero set of all these polynomials we obtain a map $\Phi$ from a $p(d)$-dimensional projective plane into $\mathcal{Z}_{n}\left(S^{n+1} ; \mathbb{Z}_{2}\right)$. Crofton formula implies that the zero-set of each of these polynomials has volume at most $\omega_{n} d$, where $\omega_{n}$ is the volume of a unit $n$-sphere. Thus, for every $\theta \in \mathbb{R} \mathbb{P}^{p(d)}, \mathbf{M}(\Phi(\theta))$ is at most a multiple of $p(d)^{\frac{1}{n+1}}$.

\subsection{Application: Existence of infinitely many minimal hyper-} surfaces. The existence of minimal hypersurfaces has been a central question in Differential Geometry. For instance, Poincaré [38] asked in 1905 whether every closed Riemann surface always admits a closed geodesic.

If the surface is not simply connected then we can minimize length in a nontrivial homotopy class and produce a closed geodesic. At the end of Section 1.1 we mentioned that, in the case of a 2 -sphere with any given metric, the question was settled by Birkhoff.

Later, in a remarkable work, Lusternik and Schnirelmann [29] showed that every metric on a 2-sphere admits three simple (embedded) closed geodesics (see also $[\mathbf{5}, \mathbf{1 6}, \mathbf{2 5}, \mathbf{2 8}, \mathbf{4 2}]$ ). This suggests the question of whether we can find an infinite number of geometrically distinct closed geodesics in any closed surface. It is not hard to find infinitely many closed geodesics when the genus of the surface is positive.

The case of the sphere was finally settled by Franks [14] and Bangert [6] (see also [23]). Their works combined imply that every metric on a twosphere admits an infinite number of closed geodesics.

Likewise, one can ask whether every closed Riemannian manifold admits a closed minimal hypersurface. We already saw that one of the consequences of the Almgren-Pitts Theory was to show that every compact Riemannian $(n+1)$-manifold contains a closed embedded minimal hypersurface with singular set of Hausdorff codimension at least 7 .

Motivated by these results, Yau conjectured in [46] (first problem in the Minimal Surfaces section) that every compact Riemannian three-manifold admits an infinite number of smooth, closed, immersed minimal surfaces.

Using the Gromov-Guth families we show

Theorem C. Let $(M, g)$ be a compact Riemannian manifold of dimension $(n+1)$, with $2 \leq n \leq 6$ and positive Ricci curvature. 
Then $M$ contains an infinite number of distinct smooth, closed, embedded, minimal hypersurfaces.

The idea behind the proof of Theorem $\mathrm{C}$ is the following.

Consider the sequence of $p$-widths $\left\{\omega_{p}(M)\right\}_{p \in \mathbb{N}}$. The sequence is nondecreasing and we use Lusternik-Schnirelmann theory to show that if $\omega_{p}(M)=$ $\omega_{p+1}(M)$ then there are infinitely many embedded minimal hypersurfaces. Thus we can assume that the sequence $\left\{\omega_{p}(M)\right\}_{p \in \mathbb{N}}$ is strictly increasing.

We then argue by contradiction and assume that there exist only finitely many smooth, closed, embedded minimal hypersurfaces. In this case we use the positive Ricci curvature combined with the Min-max Theorem to show that each min-max volume $\omega_{p}(M)$ must be achieved by a connected, closed, embedded minimal hypersurface with some integer multiplicity. Finally, we use this and the fact that $\omega_{p}(M)$ is strictly increasing to show that $\omega_{p}(M)$ must grow linearly in $p$. This is in contradiction with the sublinear growth of $\omega_{p}(M)$ in $p$ given by the Gromov and Guth's Theorem.

Acknowledgments. The first author was partly supported by CNPqBrazil and FAPERJ. The second author was partly supported by Marie Curie IRG Grant and ERC Start Grant.

Both authors are grateful to Ezequiel Rodrigues and Nicolau Sarquis for carefully reading this manuscript and for their suggestions.

\section{Almgren-Pitts Min-max theory I}

Let $(M, g)$ be an orientable compact Riemannian $(n+1)$-manifold. We assume $M$ is isometrically embedded in $\mathbb{R}^{L}$ and we denote by $B_{r}(p)$ the open geodesic ball in $M$ of radius $r$ and center $p \in M$.

We now collect some key notions in Geometric measure theory. For an introduction to the subject we recommend $[\mathbf{3 5}]$ and $[\mathbf{4 1}]$.

2.1. Geometric measure theory. In what follows, the group $G$ will always be either $\mathbb{Z}$ or $\mathbb{Z}_{2}$. The Min-max theory is set up using the following spaces:

- the space $\mathbf{I}_{k}(M ; G)$ of $k$-dimensional integral currents in $\mathbb{R}^{L}$ with support contained in $M$ and coefficients in $G$;

- the space $\mathcal{Z}_{k}(M ; G)$ of integral currents $T \in \mathbf{I}_{k}(M ; G)$ with $\partial T=0$ and coefficients in $G$;

- the closure $\mathcal{V}_{k}(M)$, in the weak topology, of the space of $k$-dimensional rectifiable varifolds in $\mathbb{R}^{L}$ with support contained in $M$. The space of integral rectifiable $k$-dimensional varifolds with support contained in $M$ is denoted by $\mathcal{I} \mathcal{V}_{k}(M)$.

When $G=\mathbb{Z}$, we use simply $\mathbf{I}_{k}(M)$ and $\mathcal{Z}_{k}(M)$.

Informally, $\mathbf{I}_{k}(M)$ is a space that contains all smooth $k$-dimensional submanifolds with smooth $(k-1)$-dimensional boundary, that is closed under addition and multiplication by integers, and that is compact assuming uniform bounds on the $k$-dimensional volume and $(k-1)$-dimensional 
boundary volume. Moreover, the support of $T \in \mathbf{I}_{k}(M)$ has a well defined $k$-dimensional tangent plane almost everywhere and, denoting by $D^{k}\left(\mathbb{R}^{L}\right)$ the space of smooth $k$-forms with compact support, one can integrate $k$ forms on $T$ and the map $\phi \in \mathcal{D}^{k}\left(\mathbb{R}^{L}\right) \mapsto T(\phi)$ defines a linear functional. Finally, $\partial T$ is well defined, belongs to $\mathcal{Z}_{k-1}(M)$ and $\partial T(\phi)=T(d \phi)$ for all $\phi$ in $\mathcal{D}^{k-1}\left(\mathbb{R}^{L}\right)$.

Given a $C^{1}$ map $f: M \rightarrow M$ and $T \in \mathbf{I}_{k}(M)$, then $f_{\#}(T) \in \mathbf{I}_{k}(M)$ is such that

$$
f_{\#}(T)(\phi)=T\left(f^{*} \phi\right) \quad \text { for all } \phi \in \mathcal{D}^{k}\left(\mathbb{R}^{L}\right) .
$$

Varifolds were introduced by Almgren in [4]. They are defined as Radon measure on the Grassmanian bundle of $k$-planes in $\mathbb{R}^{L}, G_{k}\left(\mathbb{R}^{L}\right)$, and the reader can see their properties in $[\mathbf{4 1}]$. The Radon measure associated to $V \in \mathcal{V}_{k}(M)$ is denoted by $\|V\|$. Varifolds have a well defined notion of first variation of area [41, Section 39] and so stationary varifolds are those for which the first variation of area is zero for variations with compact support. They provide a suitable generalization of minimal submanifolds. Rectifiable $k$-varifolds are those which have tangent planes defined almost everywhere and provide an alternative way to generalize the concept of a $k$-dimensional submanifold.

Every $T \in \mathbf{I}_{k}(M)$ has a $k$-rectifiable varifold with integer coefficients $|T| \in \mathcal{I V}_{k}(M)$ naturally associated to it [41, Section 27.1]. The Radon measure associated to $|T| \in \mathcal{I V}_{k}(M)$ is denoted by $\|T\|$ (see $[\mathbf{4 1}$, Section $26.8])$.

The above spaces come with several relevant metrics.

The mass of $T \in \mathbf{I}_{k}(M)$ is defined as

$$
\mathbf{M}(T)=\sup \left\{T(\phi): \phi \in \mathcal{D}^{k}\left(\mathbb{R}^{L}\right),\|\phi\| \leq 1\right\},
$$

where $\|\phi\|$ denotes the comass norm of $\phi$, and is the analogue of $k$-dimensional volume. It induces the metric $\mathbf{M}(S, T)=\mathbf{M}(S-T)$ on $\mathbf{I}_{k}(M)$. We naturally have $\|T\|(M)=\mathbf{M}(T)$.

A property we will use quite often is that open sets $A \subset M$ with finite perimeter belong to $\mathbf{I}_{n+1}(M)$ and $\partial A \in \mathcal{Z}_{n}(M)$ has finite mass (see $[\mathbf{4 1}$, Remark 27.2]).

The flat metric is defined by

$\mathcal{F}(S, T)=\inf \left\{\mathbf{M}(P)+\mathbf{M}(Q): S-T=P+\partial Q, P \in \mathbf{I}_{k}(M), Q \in \mathbf{I}_{k+1}(M)\right\}$,

for $S, T \in \mathbf{I}_{k}(M)$. Informally, $T, S \in \mathcal{Z}_{k}(M)$ very close to each other in the flat metric means that $T-S$ is the boundary of $Q \in \mathbf{I}_{k+1}(M)$ with very small mass. We also use $\mathcal{F}(T)=\mathcal{F}(T, 0)$ and one has that

$$
\mathcal{F}(T) \leq \mathbf{M}(T) \quad \text { for all } T \in \mathbf{I}_{k}(M) .
$$

The $\mathbf{F}$-metric on $\mathcal{V}_{k}(M)$ is defined in Pitts book [37, page 66] as:

$$
\begin{array}{r}
\mathbf{F}(V, W)=\sup \left\{V(f)-W(f): f \in C_{c}\left(G_{k}\left(\mathbb{R}^{L}\right)\right),\right. \\
|f| \leq 1, \operatorname{Lip}(f) \leq 1\},
\end{array}
$$


for $V, W \in \mathcal{V}_{k}(M)$. Here $C_{c}\left(G_{k}\left(\mathbb{R}^{L}\right)\right)$ denotes the space of all real-valued continuous functions with compact support defined on $G_{k}\left(\mathbb{R}^{L}\right)$. The F-metric induces the varifold weak topology on $\mathcal{V}_{k}(M)$.

The $\mathbf{F}$-metric on $\mathbf{I}_{k}(M)$ is defined by

$$
\mathbf{F}(S, T)=\mathcal{F}(S-T)+\mathbf{F}(|S|,|T|)
$$

and one has

$$
\mathbf{F}(|S|,|T|) \leq 2 \mathbf{M}(S-T) \quad \text { for every } S, T \in \mathbf{I}_{k}(M) .
$$

We assume that $\mathbf{I}_{k}(M)$ and $\mathcal{Z}_{k}(M)$ both have the topology induced by the flat metric. When endowed with the topology of the mass norm, these spaces will be denoted by $\mathbf{I}_{k}(M ; \mathbf{M})$ and $\mathcal{Z}_{k}(M ; \mathbf{M})$, respectively. If endowed with the $\mathbf{F}$-metric, we will denote them by $\mathbf{I}_{k}(M ; \mathbf{F})$ and $\mathcal{Z}_{k}(M ; \mathbf{F})$, respectively. The space $\mathcal{V}_{k}(M)$ is considered with the weak topology of varifolds.

An important fact in the theory is that the mass is only lower semicontinuous in the flat topology. The loss of mass in the limit is illustrated with the following standard example: let

$$
Q_{i}=\left\{(x, y) \in \mathbb{R}^{2}: 0 \leq x \leq 1,0 \leq y \leq i^{-1}\right\} .
$$

Then $\partial Q_{i}$ tends to zero in the flat topology but $\mathbf{M}\left(\partial Q_{i}\right)$ tends to 2 . The mass is continuous with respect to the $\mathbf{F}$-metric and the mass norm.

Following the example given above, $\partial Q_{i}$ tends to $2([0,1] \times\{0\})$ in the varifold sense, converges to zero in flat topology, and has no convergent subsequence in the mass norm because $\mathbf{M}\left(\partial\left(Q_{i}-Q_{j}\right)\right) \geq 1$ if $i \neq j$. Another simple example is to consider $T_{i}=[0,1] \times\{1 / i\}$. Then $T_{i}$ converges to $[0,1] \times\{0\}$ in the flat topology and in the varifold topology but does not converge to $T=[0,1] \times\{0\}$ in the mass topology because $\mathbf{M}\left(T_{i}-T\right)=2$ for all $i$.

When $G=\mathbb{Z}_{2}, \mathbf{I}_{k}\left(M ; \mathbb{Z}_{2}\right)$ is defined via an equivalence relation where if $T-S=2 Q, T, S, Q \in \mathbf{I}_{k}(M)$ then $T \equiv S$ (see [12, Section 4.2.26] for definition). Intuitively, this corresponds to allow submanifolds that are nonorientable. All the concepts we mentioned for $\mathbf{I}_{k}(M)$ and $\mathcal{Z}_{k}(M)$ can be extended to $\mathbf{I}_{k}\left(M ; \mathbb{Z}_{2}\right)$ and $\mathcal{Z}_{k}\left(M ; \mathbb{Z}_{2}\right)$ as well.

The following lemma will be useful.

2.2. Lemma. Let $\mathcal{S}$ be a compact subset of $\mathcal{Z}_{k}(M ; G)$ with respect to the $\mathbf{F}$-metric. For every $\varepsilon>0$ there is $\delta>0$ so that for every $S \in \mathcal{S}$ and $T \in \mathcal{Z}_{k}(M)$

$$
\mathbf{M}(T)<\mathbf{M}(S)+\delta \text { and } \mathcal{F}(T-S) \leq \delta \Rightarrow \mathbf{F}(S, T) \leq \varepsilon .
$$

Proof. In [37, page 68], it is observed that $\lim _{i \rightarrow \infty} \mathbf{F}\left(S, T_{i}\right)=0$ if and only if $\lim _{i \rightarrow \infty} \mathbf{M}\left(T_{i}\right)=\mathbf{M}(S)$ and $\lim _{i \rightarrow \infty} \mathcal{F}\left(S-T_{i}\right)=0$, for $T_{i}, S \in$ $\mathcal{Z}_{k}(M ; G)$. The lemma then follows from the continuity property of the mass functional and the compactness of $\mathcal{S}$ in $\mathcal{Z}_{k}(M ; G)$ with respect to the $\mathbf{F}$ metric, via a standard finite covering argument. 
The above lemma implies that if $T_{i} \in \mathcal{Z}_{k}(M ; G)$ tends to $T \in \mathcal{Z}_{k}(M ; G)$ in the flat topology and $\mathbf{M}\left(T_{i}\right)$ tends to $\mathbf{M}(T)$, then $T_{i}$ tends to $T$ in the F-metric.

Finally, with $\nu$ either the flat, mass, or F-metric, we define

$$
\mathbf{B}_{r}^{\nu}(T)=\left\{S \in \mathcal{Z}_{k}(M ; G): \nu(T, S)<r\right\} .
$$

Given $\mathcal{A}, \mathcal{B} \subset \mathcal{V}_{k}(M)$, we also define

$$
\mathbf{F}(\mathcal{A}, \mathcal{B})=\inf \{\mathbf{F}(V, W): V \in \mathcal{A}, W \in \mathcal{B}\} .
$$

2.3. Continuous Min-max Theorem. $X$ denotes a cubical subcomplex of some $m$-dimensional cube $I^{m}$. The group $G$ is either $\mathbb{Z}$ or $\mathbb{Z}_{2}$.

For the remainder of this notes, each map into $\mathcal{Z}_{n}(M ; G)$ that we consider is assumed to have image contained in $\left\{T \in \mathcal{Z}_{n}(M ; G): \mathbf{M}(T) \leq L\right\}$ for some $L>0$.

2.4. Homotopy notions. Consider two continuous maps in the mass topology $\Phi_{1}, \Phi_{2}: X \rightarrow \mathcal{Z}_{n}(M ; \mathbf{M} ; G)$. We say $\Phi_{1}$ is homotopic with $\Phi_{2}$ if we can find a continuous map in the mass topology

$$
H:[0,1] \times X \rightarrow \mathcal{Z}_{n}(M ; \mathbf{M} ; G)
$$

so that

$$
H(0, x)=\Phi_{1}(x) \quad \text { and } \quad H(1, x)=\Phi_{2}(x) \text { for all } x \in X .
$$

The relation "is homotopic with" is an equivalence relation on the set of all continuous maps $\Phi: X \rightarrow \mathcal{Z}_{n}(M ; \mathbf{M} ; G)$ and we call such an equivalence class a $(X, \mathbf{M})$-homotopy class of mappings into $\mathcal{Z}_{n}(M ; \mathbf{M} ; G)$. The set of all such homotopy classes is denoted by $\left[X, \mathcal{Z}_{n}(M ; \mathbf{M} ; G)\right]$ or, in case $X$ is a $k$-dimensional sphere, also by $\pi_{k}\left(\mathcal{Z}_{n}(M ; \mathbf{M} ; G)\right)$. We denote by $[\Phi]_{\mathbf{M}}$ the equivalence class of $\Phi$.

The width of $\Pi_{\mathbf{M}} \in\left[X, \mathcal{Z}_{n}(M ; \mathbf{M} ; G)\right]$ is defined as

$$
\mathbf{L}\left(\Pi_{\mathbf{M}}\right)=\inf _{\Psi \in \Pi_{\mathbf{M}}} \sup \{\mathbf{M}(\Psi(x)): x \in X\} .
$$

Given a sequence $\left\{\Phi_{i}\right\}_{i \in \mathbb{N}} \subset \Pi_{\mathbf{M}}$ we denote

$$
\mathbf{L}\left(\left\{\Phi_{i}\right\}_{i \in \mathbb{N}}\right)=\limsup _{i \rightarrow \infty} \sup \left\{\mathbf{M}\left(\Phi_{i}(x)\right): x \in X\right\}
$$

and the critical set $\mathbf{C}\left(\left\{\Phi_{i}\right\}_{i \in \mathbb{N}}\right)$ is denoted by

$$
\begin{array}{r}
\mathbf{C}\left(\left\{\Phi_{i}\right\}_{i \in \mathbb{N}}\right)=\left\{V: V=\lim _{j \rightarrow \infty}\left|\Phi_{i_{j}}\left(x_{j}\right)\right|\right. \text { as varifolds, for some increasing } \\
\text { sequence } \left.\left\{i_{j}\right\}_{j \in \mathbb{N}} \text { and } x_{j} \in X \text { and }\|V\|(M)=\mathbf{L}\left(\left\{\Phi_{i}\right\}_{i \in \mathbb{N}}\right)\right\}
\end{array}
$$

We say $\left\{\Phi_{i}\right\}_{i \in \mathbb{N}} \subset \Pi_{\mathbf{M}}$ is an optimal sequence if $\mathbf{L}\left(\left\{\Phi_{i}\right\}_{i \in \mathbb{N}}\right)=\mathbf{L}\left(\Pi_{\mathbf{M}}\right)$.

The Pitts Min-max theorem can be stated as follows 
2.5. Theorem. Assume $2 \leq n \leq 6$.

Consider $\Pi_{\mathbf{M}} \in\left[X, \mathcal{Z}_{n}(M ; \mathbf{M} ; G)\right]$ such that $\mathbf{L}\left(\Pi_{\mathbf{M}}\right)>0$.

There is a stationary integral varifold $\Sigma$, whose support is a smooth embedded minimal hypersurface, such that

$$
\|\Sigma\|(M)=\mathbf{L}\left(\Pi_{\mathbf{M}}\right) .
$$

Moreover,

- if $\left\{\Phi_{i}\right\}_{i \in \mathbb{N}}$ is an optimal sequence then $\Sigma$ can be chosen so that $\Sigma \in \mathbf{C}\left(\left\{\Phi_{i}\right\}_{i \in \mathbb{N}}\right)$ and

- we can choose an optimal sequence $\left\{\Psi_{i}\right\}_{i \in \mathbb{N}}$ so that every element in $\mathbf{C}\left(\left\{\Psi_{i}\right\}_{i \in \mathbb{N}}\right)$ is a stationary varifold.

This theorem was proven initially by Pitts for $2 \leq n \leq 5$ and later Schoen and Simon [40] extended the result to $n \geq 6$, in which case the minimal hypersurface has a singular set of Hausdorff codimension 7. The regularity theory was only done when $G=\mathbb{Z}$ but, as it was proven in Theorem 2.11 of $[\mathbf{3 2}]$, it also extends to $G=\mathbb{Z}_{2}$.

In rigor, Theorem 2.5 is not stated in those terms in Pitts book [37], but it does follow from the work developed there combined with the interpolation results proven in $[\mathbf{3 1}]$. We explain how this is done in Section 11.

Pitts chose to work with the mass topology on $\mathcal{Z}_{n}(M ; G)$ because, besides the mass being continuous (unlike the flat metric), it can easily be localized (unlike the F-metric), making it ideal for area comparisons, cut and paste arguments, and thus, regularity theory.

The disadvantage of using the mass topology is that even the simplest family, such has

$$
\Phi:[0, \pi] \rightarrow \mathcal{Z}_{2}\left(S^{3}\right), \quad \Phi(t)=\partial B_{t}(p),
$$

is continuous with respect to the $\mathbf{F}$-metric but not with respect to the mass topology. This issue is overcome by approximating maps like the one just given above by continuous maps in the mass topology. In order to state the precise theorem we need to introduce one more definition.

2.6. Mass concentration. We say that a continuous map in the flat topology $\Phi: X \rightarrow \mathcal{Z}_{n}(M ; G)$ has no concentration of mass if

$$
\varlimsup_{r \rightarrow 0} \sup \left\{\|\Phi(x)\|\left(B_{r}(p)\right): x \in X, p \in M\right\}=0 .
$$

2.7. Lemma. If $\Phi: X \rightarrow \mathcal{Z}_{n}(M ; \mathbf{M} ; G)$ is continuous in the mass norm, $\Phi$ has no concentration of mass.

Proof. Because $\Phi$ is continuous in the mass norm,

$$
G: X \times M \times[0,1] \rightarrow[0, \infty], \quad G(x, p, r)=\|\Phi(x)\|\left(B_{r}(p)\right)
$$

is continuous with $G(X, M, 0)=\{0\}$. This suffices to prove the result.

For the purpose of applications, all maps we have encountered so far have no concentration of mass. 
2.8. Example: Let $C_{i}$ be a disjoint union of circles in $\left\{x^{2}+y^{2} \leq 1 / i\right\}$ with total length 1 . One can construct $\Phi:[0,1] \rightarrow \mathcal{Z}_{1}\left(\mathbb{R}^{2}\right)$ continuous in the flat topology with $\Phi(1 / i)=C_{i}$ for all $i \in \mathbb{N}$. The map $\Phi$ has

$$
\varlimsup_{r \rightarrow 0} \sup \left\{\|\Phi(x)\|\left(B_{r}(p)\right): x \in[0,1], p \in \mathbb{R}^{2}\right\}=1
$$

and so it has concentration of mass.

Note that $C_{i}$ tends to zero in the flat topology while in the $\mathbf{F}$-topology, $\left|C_{i}\right|$ tends to a non-zero varifold $C \in \mathcal{V}_{1}\left(\mathbb{R}^{2}\right)$, which is supported at the origin. In particular, $C$ is not a 1-rectifiable varifold.

All the homotopic notions considered in Section 2.4 extend straightforwardly to the flat topology. Given a continuous map in the flat topology $\Phi: X \rightarrow \mathcal{Z}_{n}(M ; G)$ with no concentration of mass, we define $[\Phi]$ to be the set of all maps with no concentration of mass that are homotopic with $\Phi$ in the flat topology. The width of $[\Phi]$ is defined as

$$
\mathbf{L}([\Phi])=\inf _{\Psi \in[\Phi]} \sup \{\mathbf{M}(\Psi(x)): x \in X\} .
$$

We remark that given $\Phi: X \rightarrow \mathcal{Z}_{n}(M ; \mathbf{M} ; G)$ continuous in the mass topology, every element in $[\Phi]_{\mathbf{M}}$ also belongs to $[\Phi]$. We abuse notation, and say simply $[\Phi]_{\mathbf{M}} \subset[\Phi]$. Naturally we have $\mathbf{L}([\Phi]) \leq \mathbf{L}\left([\Phi]_{\mathbf{M}}\right)$.

The interpolation result we will use in order to apply Theorem 2.5 is the following.

2.9. Theorem. Let $\Phi$ be a continuous map in the flat topology $\Phi: X \rightarrow$ $\mathcal{Z}_{n}(M ; G)$ with no concentration of mass.

There is $\Pi_{\mathbf{M}}$ a $(X, \mathbf{M})$-homotopy class of mappings into $\mathcal{Z}_{n}(M ; \mathbf{M} ; G)$ such that $\Pi_{\mathbf{M}} \subset[\Phi]$ and a sequence $\left\{\Phi_{i}\right\}_{i \in \mathbb{N}} \subset \Pi_{\mathbf{M}}$ satisfying

$$
\mathbf{L}([\Phi]) \leq \mathbf{L}\left(\Pi_{M}\right) \leq \mathbf{L}\left(\left\{\Phi_{i}\right\}_{i \in \mathbb{N}}\right) \leq \sup \{\mathbf{M}(\Phi(x)): x \in X\}
$$

2.10. Continuous Min-max Theorem - Relative version. For the purpose of applications, such as the Willmore conjecture, it is important to have a min-max theorem that applies to homotopy classes relative to the boundary. For simplicity, we consider the case where the parameter space $X$ is a $m$-cube $I^{m}$ and the currents have integer coefficients, i.e., $G=\mathbb{Z}$.

We assume $\Phi_{0}: \partial I^{m} \rightarrow \mathcal{Z}_{n}(M ; \mathbf{F})$ is a continuous map in the $\mathbf{F}$-metric.

We denote by $\mathcal{E}\left(\Phi_{0}\right)$ the set of all continuous maps in the flat topology with no mass concentration $\Phi: I^{m} \rightarrow \mathcal{Z}_{n}(M)$ so that $\Phi=\Phi_{0}$ on $\partial I^{m}$.

We denote by $\mathcal{E}\left(\Phi_{0} ; \mathbf{M}\right)$ the set of all continuous maps in the $\mathbf{F}$-metric $\Phi: I^{m} \rightarrow \mathcal{Z}_{n}(M ; \mathbf{F})$ such that $\Phi$ is continuous in the mass topology when restricted to the interior of $I^{m}$ and $\Phi=\Phi_{0}$ on $\partial I^{m}$. 
2.11. Homotopy notions - Relative version. Consider two maps $\Phi_{1}, \Phi_{2} \in \mathcal{E}\left(\Phi_{0} ; \mathbf{M}\right)$. We say $\Phi_{1}$ is homotopic with $\Phi_{2}$ if $\Phi_{1}-\Phi_{2}$ is homotopic to zero in the mass topology relative to $\partial I^{m}$, i.e., we can find a continuous map in the mass topology

$$
H: I \times I^{m} \rightarrow \mathcal{Z}_{n}(M ; \mathbf{M})
$$

so that $H\left(I \times \partial I^{m}\right)=\{0\}$,

$$
H(0, x)=\left(\Phi_{1}-\Phi_{2}\right)(x) \text { and } H(1, x)=0 \text { for all } x \in I^{m} .
$$

The relation "is homotopic with" is an equivalence relation on $\mathcal{E}\left(\Phi_{0} ; \mathbf{M}\right)$ and we call such an equivalence class a $(X, \mathbf{M})$-homotopy class of mappings into $\left(\mathcal{Z}_{n}(M ; \mathbf{M}), \Phi_{0}\right)$. The set of all such homotopy classes is denoted by $\pi_{m}\left(\mathcal{Z}_{n}(M ; \mathbf{M}), \Phi_{0}\right)$ and we denote by $[\Phi]_{\mathbf{M}}$ the equivalence class of $\Phi \in$ $\mathcal{E}\left(\Phi_{0} ; \mathbf{M}\right)$.

The remaining notions, such as width, critical set and so on, can be extended in an obvious way. For the sake of simplicity, we use the same notation as in Section 2.4 because it will be clear from the context whether we are using relative homotopies or not.

The relative version of the min-max theorem can now be stated.

2.12. Theorem. Assume $2 \leq n \leq 6$.

Consider $\Pi_{\mathbf{M}} \in \pi_{m}\left(\mathcal{Z}_{n}(M ; \mathbf{M}), \Phi_{0}\right)$ such that $\mathbf{L}\left(\Pi_{\mathbf{M}}\right)>\sup _{x \in \partial I^{m}} \mathbf{M}\left(\Phi_{0}(x)\right)$.

There exists a stationary integral varifold $\Sigma$, whose support is a smooth embedded minimal hypersurface, such that

$$
\|\Sigma\|(M)=\mathbf{L}\left(\Pi_{\mathbf{M}}\right)
$$

Moreover,

- if $\left\{\Phi_{i}\right\}_{i \in \mathbb{N}}$ is an optimal sequence then $\Sigma$ can be chosen so that $\Sigma \in \mathbf{C}\left(\left\{\Phi_{i}\right\}_{i \in \mathbb{N}}\right)$ and

- we can choose an optimal sequence $\left\{\Psi_{i}\right\}_{i \in \mathbb{N}}$ so that every element in $\mathbf{C}\left(\left\{\Psi_{i}\right\}_{i \in \mathbb{N}}\right)$ is a stationary varifold.

In Section 11 we explain how this theorem follows from the min-max theorem in $[\mathbf{3 7}]$.

In order for Theorem 2.12 be of use, we need to approximate maps in $\mathcal{E}\left(\Phi_{0}\right)$ by maps in $\mathcal{E}\left(\Phi_{0} ; \mathbf{M}\right)$.

Given $\Phi \in \mathcal{E}\left(\Phi_{0}\right)$, we denote by $[\Phi]$ the equivalence class of maps $\Psi \in$ $\mathcal{E}\left(\Phi_{0}\right)$ that are homotopic to $\Phi$ in the flat topology relative to $\partial I^{m}$. The width of $[\Phi]$ is defined as

$$
\mathbf{L}([\Phi])=\inf _{\Psi \in[\Phi]} \sup \left\{\mathbf{M}(\Psi(x)): x \in I^{m}\right\} .
$$

We remark that given $\Phi \in \mathcal{E}\left(\Phi_{0} ; \mathbf{M}\right)$ we have, with an obvious abuse of notation, $[\Phi]_{\mathbf{M}} \subset[\Phi]$ and so $\mathbf{L}([\Phi]) \leq \mathbf{L}\left([\Phi]_{\mathbf{M}}\right)$.

The interpolation result we will use in order to apply Theorem 2.12 is the following. 
2.13. Theorem. Choose $\Phi \in \mathcal{E}\left(\Phi_{0}\right)$.

There is $\Pi_{\mathbf{M}}$ a $\left(I^{m}, \mathbf{M}\right)$-homotopy class of mappings into $\left(\mathcal{Z}_{n}(M ; \mathbf{M}), \Phi_{0}\right)$ such that $\Pi_{\mathbf{M}} \subset[\Phi]$ and a sequence $\left\{\Phi_{i}\right\}_{i \in \mathbb{N}} \subset \Pi_{\mathbf{M}}$ satisfying

$$
\mathbf{L}([\Phi]) \leq \mathbf{L}\left(\Pi_{\mathbf{M}}\right) \leq \mathbf{L}\left(\left\{\Phi_{i}\right\}_{i \in \mathbb{N}}\right) \leq \sup \left\{\mathbf{M}(\Phi(x)): x \in I^{m}\right\} .
$$

2.14. Almgren isomorphism. In [2], Almgren gave an optimal criterion to determine when a continuous map in the flat topology $\Phi:[0,1] \rightarrow$ $\mathcal{Z}_{n}(M)$ with $\Phi(0)=\Phi(1)=0$ cannot be homotoped to a constant map relative to the boundary of $[0,1]$. Almgren's result extends straightforwardly to the case where the currents have $\mathbb{Z}_{2}$ coefficients or the maps are parametrized by the unit circle $S^{1}$.

We describe Almgren's construction. He showed the existence [2, Theorem 2.4] of a constant $\nu_{M}$ and $\rho=\rho(M) \geq 1$ so that if $S, T \in \mathcal{Z}_{n}(M ; G)$ have $\mathcal{F}(S-T)<\nu_{M}$, then one can find a unique $Q \in \mathbf{I}_{n+1}(M ; G)$ so that

$$
\partial Q=S-T \quad \text { and } \quad \mathbf{M}(Q) \leq \rho \mathcal{F}(S-T) .
$$

Given a continuous map in the flat topology $\Phi: S^{1} \rightarrow \mathcal{Z}_{n}(M ; G)$, choose $k$ sufficiently large so that,

$$
\mathcal{F}\left(\Phi\left(e^{2 \pi i x}\right), \Phi\left(e^{2 \pi i y}\right)\right) \leq \nu_{M} \quad \text { for all } x, y \text { with }|x-y| \leq 3^{-k} .
$$

Hence, for $j=0, \ldots, 3^{k}-1$, there is $Q_{j} \in \mathbf{I}_{n+1}(M ; G)$ so that

$\partial Q_{j}=\Phi\left(e^{2 \pi i a_{j+1}}\right)-\Phi\left(e^{2 \pi i a_{j}}\right) \quad$ and $\quad \mathbf{M}\left(Q_{j}\right) \leq \rho \mathcal{F}\left(\Phi\left(e^{2 \pi i a_{j+1}}\right)-\Phi\left(e^{2 \pi i a_{j}}\right)\right)$,

where $a_{j}=j 3^{-k}$. Hence $\sum_{j=0}^{3^{k}-1} A_{j} \in \mathcal{Z}_{n+1}(M ; G)$ and therefore it defines a homology class (see [12, Section 4.4]):

$$
F_{M}(\Phi)=\left[\sum_{j=0}^{3^{k}-1} A_{j}\right] \in H_{n+1}(M ; G)
$$

In [2], Almgren showed that $F_{M}(\Phi)$ does not depend on how $k$ was chosen provided (4) is satisfied and that if $\Psi$ is homotopic to $\Phi$ in the flat topology, then $F_{M}(\Phi)=F_{M}(\Psi)$. Hence, he obtains a map,

$$
F_{M}: \pi_{1}\left(\mathcal{Z}_{n}(M ; G)\right) \rightarrow H_{n+1}(M ; G), \quad[\Phi]_{\mathcal{F}} \mapsto F_{M}(\Phi),
$$

where $[\Phi]_{\mathcal{F}}$ denotes the equivalence class of all maps homotopic to $\Phi$ in the flat topology. In Section 3 of [2], Almgren shows that $F_{M}$ is an isomorphism when restricted to connected components of $\mathcal{Z}_{n}(M ; G)$. In particular, $F_{M}(\Phi)=0$ if and only if $\Phi$ is homotopic in the flat topology to a constant map.

We call the map $F_{M}$ the Almgren isomorphism.

An analogous discussion holds for continuous maps in the flat topology $\Phi:[0,1] \rightarrow \mathcal{Z}_{n}(M ; G)$ with $\Phi(0)=\Phi(1)=0$. Namely, denoting by $[\Phi]_{\mathcal{F}}$ the 
equivalence class of maps homotopic to $\Phi$ in the flat topology relative to the boundary of $[0,1]$, one also constructs an isomorphism

$$
F_{M}: \pi_{1}\left(\mathcal{Z}_{n}(M ; G),\{0\}\right) \rightarrow H_{n+1}(M ; G)
$$

and $F_{M}\left([\Phi]_{\mathcal{F}}\right)=0$ if and only if $\Phi$ is homotopic to zero in the flat topology relative to the boundary of $[0,1]$.

2.15. Sweepout. A continuous map in the flat topology $\Phi: S^{1} \rightarrow$ $\mathcal{Z}_{n}(M ; G)$ or $\Phi:[0,1] \rightarrow \mathcal{Z}_{n}(M ; G)$ with $\Phi(0)=\Phi(1)=0$, is called a sweepout if $F_{M}\left([\Phi]_{\mathcal{F}}\right) \neq 0$ in $H^{n+1}(M, G)$. If $F_{M}\left([\Phi]_{\mathcal{F}}\right)=0$ the map is called trivial.

2.16. Remark: In Theorem 4.6 of [37], Pitts showed that the groups

$$
\pi_{1}\left(\mathcal{Z}_{n}(M ; G),\{0\}\right), \quad \pi_{1}\left(\mathcal{Z}_{n}(M ; \mathbf{M} ; G),\{0\}\right), \quad \text { and } \quad H_{n+1}(M ; G)
$$

are all naturally isomorphic via the Almgren isomorphism. Hence if the map $\Phi:[0,1] \rightarrow \mathcal{Z}_{n}(M ; \mathbf{M} ; G)$ with $\Phi(0)=\Phi(1)=0$ is homotopic to zero in the flat topology relative to the boundary of $[0,1]$, then $\Phi$ is also homotopic to zero in the mass topology relative to the boundary of $[0,1]$.

2.17. Lemma. Let $\mathcal{T}$ be a finite set of $\mathcal{Z}_{n}(M ; G)$. There is $\varepsilon>0$ depending on $\mathcal{T}$ so that continuous maps in the flat topology

$$
\Phi: S^{1} \rightarrow \mathcal{Z}_{n}(M ; G) \quad \text { or } \quad \Phi:[0,1] \rightarrow \mathcal{Z}_{n}(M ; G) \text { with } \Phi(0)=\Phi(1)=0
$$

such that

$$
\Phi\left(S^{1}\right) \subset \mathbf{B}_{\varepsilon}^{\mathcal{F}}(\mathcal{T}) \quad \text { or } \quad \Phi([0,1]) \subset \mathbf{B}_{\varepsilon}^{\mathcal{F}}(\mathcal{T})
$$

respectively, are trivial.

Proof. We consider first the case where $\mathcal{T}=\{0\}$. From either Proposition 3.5 of $[\mathbf{3 2}]$ or Theorem 8.2 of [2] in the relative case, we see the existence of $\varepsilon_{0}$ so that if either

$$
\Phi\left(S^{1}\right) \subset \mathbf{B}_{\varepsilon_{0}}^{\mathcal{F}}(0) \quad \text { or } \quad \Phi([0,1]) \subset \mathbf{B}_{\varepsilon}^{\mathcal{F}}(0),
$$

respectively, then $\Phi$ is homotopic to zero and thus trivial.

In the general case consider $d=\min \{\mathcal{F}(S, T): S, T \in \mathcal{T}, S \neq T\}$ and set $\varepsilon=\min \left\{\varepsilon_{0}, d / 3\right\}$. Then

$$
\Phi\left(S^{1}\right) \subset \mathbf{B}_{\varepsilon}^{\mathcal{F}}(T) \quad \text { or } \quad \Phi([0,1]) \subset \mathbf{B}_{\varepsilon}^{\mathcal{F}}(T)
$$

for some $T \in \mathcal{T}$. Thus $\Phi$ is homotopic to a constant map (from the first case considered) and thus trivial. 
2.18. Standard sweepout. With $p \in S^{3}$ fixed, consider

$$
\Phi:[0,1] \rightarrow \mathcal{Z}_{2}\left(S^{3}\right), \quad \Phi(t)=\partial B_{t \pi}(p) .
$$

We explain in detail how Theorem 2.12, Theorem 2.13, and Lemma 2.17, are used to show that

$$
\mathbf{L}([\Phi])=4 \pi=\sup _{x \in[0,1]} \mathbf{M}(\Phi(x))
$$

where we recall that $[\Phi]$ is the equivalence class of continuous maps with no concentration of mass that are homotopic with $\Phi$ in the flat topology relative to the boundary of $[0,1]$.

We have $F_{M}\left([\Phi]_{\mathcal{F}}\right)=\left[S^{3}\right]$ and so $\Phi$ is a sweepout. Thus, using the fact that $[\Phi] \subset[\Phi]_{\mathcal{F}}$, we obtain from Lemma 2.17 that

$$
4 \pi=\sup _{x \in[0,1]} \mathbf{M}(\Phi(x)) \geq \mathbf{L}([\Phi]) \geq \inf _{\Psi \in[\Phi]_{\mathcal{F}}} \sup _{x \in[0,1]} \mathbf{M}(\Psi(x))>0 .
$$

Suppose there is $\Psi \in[\Phi]$ so that $\sup _{x \in[0,1]} \mathbf{M}(\Psi(x))<4 \pi$. From Theorem 2.13 we obtain $\Pi_{\mathbf{M}}$ a $([0,1], \mathbf{M})$-homotopy class of mappings into $\left(\mathcal{Z}_{2}\left(S^{3} ; \mathbf{M}\right), 0\right)$ so that

$$
0<\mathbf{L}([\Phi]) \leq \mathbf{L}\left(\Pi_{M}\right) \leq \sup _{x \in[0,1]} \mathbf{M}(\Psi(x))<4 \pi
$$

Thus Theorem 2.12 gives the existence of a minimal surface in $S^{3}$ with area less than $4 \pi$ and this is impossible.

\section{Criterion for a 5-cycle in $\mathcal{Z}_{2}\left(S^{3}\right)$ to be nontrivial.}

In Section 2.14 we saw a criterion that determines when a continuous map in the flat topology $\Phi:[0,1] \rightarrow \mathcal{Z}_{n}(M ; G)$ cannot be deformed into a constant map. We devote this section to give a criterion under which a map $\Phi: I^{5} \rightarrow \mathcal{Z}_{2}\left(S^{3}\right)$ cannot be deformed into the space of all round spheres in $S^{3}$.

Recall that the geodesic ball in $S^{3}$ centered at $p \in S^{3}$ and with radius $r$ is denoted by $B_{r}(p)$. We use the notions of relative homotopies defined in Section 2.11 and the standard sweepout is defined in Section 2.18.

3.1. Theorem. Let $\Phi_{0}: \partial I^{5} \rightarrow \mathcal{Z}_{2}\left(S^{3} ; \mathbf{F}\right)$ be a continuous map in the F-topology such that:

(1) $\Phi_{0}(x, 0)=\Phi_{0}(x, 1)=0$ for any $x \in I^{4}$;

(2) there is a map $Q: \partial I^{4} \rightarrow S^{3}$ so that

$$
\Phi_{0}(x, t)=\partial B_{t \pi}(Q(x)) \quad \text { for all }(x, t) \in \partial I^{4} \times I
$$

(3) the map $Q: \partial I^{4} \rightarrow S^{3}$ has $\operatorname{deg}(Q) \neq 0$.

Every $\Phi \in \mathcal{E}\left(\Phi_{0}\right)$ has

$$
\mathbf{L}([\Phi])>4 \pi
$$

Before we prove Theorem 3.1 we make several comments about its hypothesis. 
Let $\mathcal{G}_{o}$ denote the set of all oriented geodesic spheres in $\mathcal{Z}_{2}\left(S^{3}\right)$. A nonzero element in $\mathcal{G}_{o}$ is uniquely given by $\partial B_{r}(p)$, where $p \in S^{3}$ and $0<r<\pi$.

One consequence of condition (2) in Theorem 3.1 is that $\Phi_{0}(x, 1 / 2)=$ $\partial B_{\pi / 2}(Q(x))$ for all $x \in \partial I^{4}$ and, given $y \in \partial I^{4}, \Phi_{0}(y, t)$ is a great sphere if and only if $t=1 / 2$.

Conditions (1) and (2) in Theorem 3.1 mean that $\Phi\left(\partial I^{5}\right) \subset \mathcal{G}_{o}$. Informally, $[\Phi]$ can be thought of as an element of the relative homotopy group $\pi_{5}\left(\mathcal{Z}_{2}\left(S^{3}\right), \mathcal{G}_{o}\right)$ and the theorem is giving conditions under which $[\Phi] \neq 0$ in $\pi_{5}\left(\mathcal{Z}_{2}\left(S^{3}\right), \mathcal{G}_{o}\right)$.

Condition (3) is crucial, as the next example shows. Consider

$$
\Phi: I^{5} \rightarrow \mathcal{Z}_{2}\left(S^{3}\right), \quad \Phi(x, t)=\partial B_{\pi t}(p),
$$

where $p$ is a fixed point in $S^{3}$. With $\Phi_{0}=\Phi_{\mid \partial I^{5}}$, we see that conditions (1) and (2) of Theorem 3.1 are satisfied but $\mathbf{L}([\Phi])=4 \pi$ because $\Phi\left(I^{5}\right) \subset \mathcal{G}_{o}$.

The sweepout requirement in condition (2) of Theorem 3.1 is also important. To see this, choose a continuous map $Q_{1}: S^{3} \rightarrow S^{3}$ with non-zero degree and set

where

$$
\tilde{\Phi}: \bar{B}^{4} \times[0,1] \rightarrow \mathcal{Z}_{2}\left(S^{3}\right)
$$

$$
\tilde{\Phi}(x, t)=\partial B_{2 \pi|x| t(1-t)}\left(Q_{1}(x /|x|)\right) \quad \text { if } x \neq 0 \quad \text { and } \Phi(0, t)=0 .
$$

With $D$ a homeomorphism from $I^{4}$ to $\bar{B}^{4}$, the map $\Phi(x, t)=\tilde{\Phi}(D(x), t)$ satisfies conditions (1) and (3) of Theorem 3.1 but $\mathbf{L}([\Phi])=4 \pi$ because $\Phi\left(I^{5}\right) \subset \mathcal{G}_{0}$.

Denote by $\mathcal{T} \subset \mathcal{V}_{2}\left(S^{3}\right)$ the set of all (unoriented) great spheres in $S^{3}$, which is homeomorphic to $\mathbb{R P}^{3}$ via $[p] \in \mathbb{R} \mathbb{P}^{3} \mapsto\left|\partial B_{\pi / 2}(p)\right|$. Hence we have a map

$$
\left|\Phi_{0}\right|: \partial I^{4} \times\{1 / 2\} \rightarrow \mathcal{T} \approx \mathbb{R P}^{3}, \quad\left|\Phi_{0}\right|(x, 1 / 2)=\left|\partial B_{\pi / 2}(Q(x))\right|
$$

whose degree is $2 \operatorname{deg}(Q)$.

The idea for the proof of Theorem 3.1 is, in general terms, the following. We argue by contradiction and assume that $\mathbf{L}([\Phi])=4 \pi$. Hence we can find $\Phi_{i} \in[\Phi]$ so that

$$
\lim _{i \rightarrow \infty} \sup _{x \in I^{5}} \mathbf{M}\left(\Phi_{i}(x)\right)=\mathbf{L}([\Phi])=4 \pi .
$$

For $\varepsilon$ sufficiently small and all $i$ sufficiently large we find a hypersurface $R(i)$ in $I^{5}$ with

$$
R(i) \subset\left\{x \in I^{5}: \mathbf{F}\left(\left|\Phi_{i}(x)\right|, \mathcal{T}\right)=\varepsilon\right\}, \quad \partial R(i) \subset \partial I^{4} \times I,
$$

and

$$
[\partial R(i)]=\left[\partial I^{4} \times\{1 / 2\}\right] \text { in } H_{3}\left(\partial I^{4} \times I, \mathbb{Z}\right) .
$$

We then use the fact that $\Phi_{i}$ coincides with $\Phi_{0}$ on $\partial I^{5}$ to perturb $\left|\Phi_{i}\right|$ on $R(i)$ and obtain a continuous map $f_{i}: R(i) \rightarrow \mathcal{T} \approx \mathbb{R P}^{3}$ so that

$$
\left(f_{i}\right)_{*}[\partial R(i)]=\left|\Phi_{0}\right|_{*}\left[\partial I^{4} \times\{1 / 2\}\right]=2 \operatorname{deg}(Q)\left[\mathbb{R} \mathbb{P}^{3}\right] \neq 0 .
$$


On the other hand,

$$
\left(f_{i}\right)_{*}[\partial R(i)]=\partial\left[f_{i}(R(i))\right]=0 \quad \text { in } H_{3}\left(\mathbb{R P}^{3}, \mathbb{Z}\right),
$$

and this is a contradiction.

3.2. Proof of Theorem 3.1. Consider the cubical complex $I(5, k)$ obtained by subdividing $I^{5}$ into 5 -cubes of side length $3^{-k}$. We abuse notation and identify a sum of $p$-cells in $I(5, k)$ with its support and with the correspondent integral current in $\mathbf{I}_{p}\left(I^{5}\right)$.

Suppose $\mathbf{L}([\Phi])=4 \pi$. Using Theorem 2.13 we choose a sequence $\left\{\Phi_{i}\right\}_{i \in \mathbb{N}}$ in $\mathcal{E}\left(\Phi_{0} ; \mathbf{M}\right)$ so that

$$
\lim _{i \rightarrow \infty} \sup _{x \in I^{5}} \mathbf{M}\left(\Phi_{i}(x)\right)=4 \pi .
$$

Fix $\varepsilon_{0}$ small to be chosen later and, for each $i \in \mathbb{N}$, choose $k_{i} \in \mathbb{N}$ so that for every 5 -cell $\alpha$ in $I\left(5, k_{i}\right)$ we have

$$
\sup \{\mathbf{F}(|\Phi(x)|,|\Phi(y)|): x, y \in \alpha\} \leq \varepsilon_{0} / 2 .
$$

Set $\mathbf{A}(i)$ to be the connected component of

$$
\left\{x \in I^{5}: \mathbf{F}\left(\left|\Phi_{i}(x)\right|, \mathcal{T}\right) \geq \varepsilon_{0} / 2\right\}
$$

that contains $I^{4} \times\{0\}$ and let $A(i) \in \mathbf{I}_{5}\left(I^{5}\right)$ be the sum of all 5-cells in $I\left(5, k_{i}\right)$ that are contained in $\mathbf{A}(i)$.

3.3. Lemma. For all $i$ sufficiently large, the support of $A(i)$ does not intersect $I^{4} \times\{1\}$.

Proof. Fix $x_{0} \in \partial I^{4}$ and consider the standard sweepout $\Psi:[0,1] \rightarrow$ $\mathcal{Z}_{2}\left(S^{3}\right)$ given by $\Psi(t)=\Phi_{0}\left(x_{0}, t\right)$.

Assume by contradiction the existence of a subsequence $\left\{x_{i_{j}}\right\}_{j \in \mathbb{N}}$ with $x_{i_{j}} \in \operatorname{supp} A\left(i_{j}\right) \cap I^{4} \times\{1\}$. Then $x_{i_{j}} \in \mathbf{A}\left(i_{j}\right)$ and so we can find a continuous path

$$
\gamma_{j}:[0,1] \rightarrow \mathbf{A}\left(i_{j}\right) \quad \text { with } \gamma_{j}(0) \in I^{4} \times\{0\} \text { and } \gamma_{j}(0) \in I^{4} \times\{1\}
$$

Note that $\Psi_{j}=\Phi_{i_{j}} \circ \gamma_{i}$ is homotopic with $\Psi$ in the flat topology relative to $\partial[0,1]$ and thus, by Remark 2.16, they all determine the same $([0,1], \mathbf{M})$ homotopy class of mappings into $\left(\mathcal{Z}_{2}\left(S^{3} ; \mathbf{M}\right), 0\right)$ that we denote by $\Pi_{\mathbf{M}}$. Moreover we also have

$$
\mathbf{L}\left(\Pi_{\mathbf{M}}\right) \leq \lim _{j \rightarrow \infty} \sup _{t \in[0,1]} \mathbf{M}\left(\Psi_{j}(t)\right) \leq \lim _{j \rightarrow \infty} \sup _{x \in I^{5}} \mathbf{M}\left(\Phi_{i_{j}}(x)\right)=4 \pi
$$

and, from Section 2.18,

$$
\lim _{j \rightarrow \infty} \sup _{t \in[0,1]} \mathbf{M}\left(\Psi_{j}(t)\right) \geq \mathbf{L}\left(\Pi_{\mathbf{M}}\right) \geq \mathbf{L}([\Psi])=4 \pi .
$$

Therefore, $\left\{\Psi_{j}\right\}_{j \in \mathbb{N}}$ is a critical sequence for $\Pi_{\mathbf{M}}$ and Theorem 2.12 implies the existence of $0 \leq t_{j} \leq 1$ so that a subsequence of $\left\{\left|\Psi_{j}\left(t_{j}\right)\right|\right\}_{j \in \mathbb{N}}$ tends to a great sphere in varifold norm. But $\mathbf{F}\left(\left|\Psi_{j}\left(t_{j}\right)\right|, \mathcal{T}\right) \geq \varepsilon_{0} / 2$ from the way $\mathbf{A}(i)$ and $\Psi_{j}$ were defined and this gives us a contradiction. 
The current $\partial A(i) \in \mathcal{Z}_{4}\left(I^{5}\right)$ can be written as the sum of 4-cells in $I\left(5, k_{i}\right)$. Thus we can decompose it as

$$
\partial A(i)=-I^{4} \times\{0\}+C(i)+R(i),
$$

where $C(i) \in \mathbf{I}_{4}\left(\partial I^{4} \times I\right)$ and $R(i)$ has the property that no 4-cell contained in $R(i)$ is contained in $\partial I^{4} \times I$.

3.4. Lemma. We have

(i) $\partial R(i) \in \mathcal{Z}_{3}\left(\partial I^{4} \times I\right)$;

(ii) $[\partial R(i)]=\left[\partial I^{4} \times\{1 / 2\}\right]$ in $H_{3}\left(\partial I^{4} \times I, \mathbb{Z}\right)$;

(iii) for all $i$ sufficiently large,

$$
x \in \operatorname{supp} R(i) \Longrightarrow \mathbf{F}\left(\left|\Phi_{i}(x)\right|, \mathcal{T}\right)<\varepsilon_{0} .
$$

Proof. From the definition of $R(i)$ we see that

$$
\partial R(i)=\partial\left(I^{4} \times\{0\}\right)-\partial C(i)=\partial I^{4} \times\{0\}-\partial C(i)
$$

and hence $\partial R(i) \in \mathcal{Z}_{3}\left(\partial I^{4} \times I\right)$. Moreover the identity above implies as well that

$$
[\partial R(i)]=\left[\partial I^{4} \times\{0\}\right]=\left[\partial I^{4} \times\{1 / 2\}\right] \quad \text { in } H_{3}\left(\partial I^{4} \times I, \mathbb{Z}\right) .
$$

To prove (iii) choose $i$ large so that Lemma 3.3 applies. Given $x \in R(i)$, there is a 4-cell $\sigma \in I\left(5, k_{i}\right)$ containing $x$ and such that $\alpha$ is not contained in $\partial I^{4} \times I \cup I^{4} \times\{0\}$. Because $\partial A(i)$ does not intersect $I^{4} \times\{1\}$, then $\alpha$ is the boundary of some 5 -cell $\alpha \in I\left(5, k_{i}\right)$ that does not belong to the support of $A(i)$. From the definition of $A(i)$ we see that this implies the existence of $y \in \alpha$ such that $y \notin \mathbf{A}(i)$, which means that $\mathbf{F}\left(\left|\Phi_{i}(y)\right|, \mathcal{T}\right)<\varepsilon_{0} / 2$. From $(7)$ we obtain that

$$
\mathbf{F}\left(\left|\Phi_{i}(x)\right|, \mathcal{T}\right) \leq \mathbf{F}\left(\left|\Phi_{i}(x)\right|, \mid \Phi_{i}(y)\right)+\mathbf{F}\left(\left|\Phi_{i}(y)\right|, \mathcal{T}\right)<\varepsilon_{0}
$$

and this implies the desired result.

On $\partial I^{4} \times I$ consider the map

$$
\hat{\Phi}_{0}: \partial I^{4} \times I \rightarrow \mathcal{T}, \quad \hat{\Phi}_{0}(x, t)=\left|\Phi_{0}(x, 1 / 2)\right|=\left|\partial B_{\pi / 2}(Q(x))\right| .
$$

3.5. Lemma. For all $i$ large, there is a continuous function

$$
f_{i}: R(i) \rightarrow \mathcal{T} \approx \mathbb{R P}^{3} \text { with } f_{i}=\hat{\Phi}_{0} \text { on } \partial R(i)
$$

Sketch of PRoOF. The lemma is proven in Lemma 9.10 of [31] and so we simply outline the main ideas.

The integral current $R(i)$ is a sum of 4 -cells in $I\left(5, k_{i}\right)$ and we denote the set of the $p$-cells in $R(i)$ or $\partial R(i)$ by $R(i)_{p}$ and $\partial R(i)_{p}$, respectively.

The idea is to first define $f_{i}$ on the 0 -cells of $R(i)$, then extend the definition to the 1-cells of $R(i)$, and proceed inductively until $f_{i}$ is defined on all of $R(i)$. 
The map $f_{i}: R(i)_{0} \rightarrow \mathcal{T}$ is defined as follows: if $x \in \partial R(i)_{0}$ we set $f_{i}(x)=\hat{\Phi}_{0}(x)$, otherwise we choose $f_{i}(x) \in \mathcal{T}$ such that

$$
\mathbf{F}\left(f_{i}(x),\left|\Phi_{i}(x)\right|\right)=\mathbf{F}\left(\left|\Phi_{i}(x)\right|, \mathcal{T}\right) .
$$

Given any $\eta$ small we can find, using (7) and Lemma 3.4 (iii), $\varepsilon_{0}$ small so that any two adjacent vertices $x, y$ in $R(i)_{0}$ have $\mathbf{F}\left(f_{i}(x), f_{i}(y)\right) \leq \eta$. The topology induced by the $\mathbf{F}$-metric on $\mathcal{T} \approx \mathbb{R P}^{3}$ coincides with the topology induced by the geodesic distance of $\mathbb{R P}^{3}$. Thus, by requiring $\eta$ to be small enough, we can ensure that $f_{i}(x), f_{i}(y)$ lie in the same geodesically convex neighborhood in $\mathbb{R P}^{3}$ whenever $x$ and $y$ are adjacent vertices in $R(i)_{0}$. Hence, if $\alpha$ is a 1-cell in $\partial R(i)_{1}$ we extend $f_{i}$ to $\alpha$ by defining it to be $\hat{\Phi}_{0}$ and if $\alpha \in R(i)_{1} \backslash \partial R(i)_{1}$ we use the fact that $f_{i}(\partial \alpha)$ is contained in the same geodesically convex neighborhood to extend $f_{i}$ continuously to $\alpha$. This can be done in a way that, for every $\alpha \in R(i)_{1}$, we still have $f_{i}(\alpha)$ contained in the same geodesically convex neighborhood of $\mathcal{T}$ and so we can proceed inductively to obtain $f_{i}$ defined on $R(i)$.

This lemma implies that, in homology,

$$
\left(\hat{\Phi}_{0}\right)_{*}[\partial R(i)]=f_{i_{*}}[\partial R(i)]=\left[f_{i \#} \partial(R(i))\right]=\partial\left[f_{i_{\#}}(R(i))\right]=0 .
$$

On the other hand using (6) and the fact that $\partial R(i)$ is homologous to $\partial I^{4} \times\{1 / 2\}$ in $\partial I^{4} \times I$ we obtain

$$
\begin{aligned}
\left(\hat{\Phi}_{0}\right)_{*}[\partial R(i)]=\left(\hat{\Phi}_{0}\right)_{*}\left[\partial I^{4} \times\right. & \{1 / 2\}] \\
& =\left|\Phi_{0}\right|_{*}\left[\partial I^{4} \times\{1 / 2\}\right]=2 \operatorname{deg}(Q)\left[\mathbb{R} \mathbb{P}^{3}\right] \neq 0 .
\end{aligned}
$$

We have reached a contradiction and thus $L([\Phi])>4 \pi$.

\section{Canonical family of surfaces}

In this section we construct maps to which we can apply Theorem 3.1. Namely we devote this section to show:

4.1. Theorem. Let $\Sigma \subset S^{3}$ be an embedded closed surface of genus $g$.

There is a continuous map in the flat topology $\Phi: I^{5} \rightarrow \mathcal{Z}_{2}\left(S^{3}\right)$ with no concentration of mass and satisfying the properties:

(1) $\Phi(x, 0)=\Phi(x, 1)=0$ for any $x \in I^{4}$;

(2) there is a map $Q: \partial I^{4} \rightarrow S^{3}$ so that

$$
\Phi(x, t)=\partial B_{t \pi}(Q(x)) \quad \text { for all }(x, t) \in \partial I^{4} \times I ;
$$

(3) $\sup \left\{\mathbf{M}(\Phi(x)): x \in I^{5}\right\} \leq \mathcal{W}(\Sigma)$;

(4) the center map $Q: \partial I^{4} \rightarrow S^{3}$ satisfies $\operatorname{deg}(Q)=g$.

The discovery of a map $\Phi$ with such good properties, namely Theorem 4.1 (3) and (4), was what made possible the proof of the Willmore conjecture. 
4.2. Canonical family. Recall that $B^{4}$ is the unit 4-ball. For each $v \in B^{4}$ consider the conformal map

$$
F_{v}: S^{3} \rightarrow S^{3}, \quad F_{v}(x)=\frac{\left(1-|v|^{2}\right)}{|x-v|^{2}}(x-v)-v .
$$

The map $F_{0}$ is the identity and, for $v \neq 0, F_{v}$ is a conformal dilation of $S^{3}$ with fixed points $v /|v|$ and $-v /|v|$.

Let $\Sigma \subset S^{3}$ be an embedded closed surface of genus $g$ and choose an open set $A$ so that $\Sigma=\partial A$. For any $v \in B^{4}$ consider $A_{v}=F_{v}(A)$ with boundary $\Sigma_{v}=F_{v}(\Sigma)$.

Define the signed distance $d_{v}: S^{3} \rightarrow \mathbb{R}$ to $\Sigma_{v} \subset S^{3}$ as

$$
d_{v}(x)=\left\{\begin{aligned}
d\left(x, \Sigma_{v}\right) & \text { if } x \notin A_{v}, \\
-d\left(x, \Sigma_{v}\right) & \text { if } x \in A_{v},
\end{aligned}\right.
$$

where $d\left(x, \Sigma_{v}\right)$ denotes the distance from $x$ to $\Sigma_{v}$.

Finally, we consider the following 5-parameter family of surfaces associated to $\Sigma$ :

$$
\Sigma_{(v, t)}=\partial\left\{x \in S^{3}: d_{v}(x)<t\right\}, \quad \text { where }(v, t) \in B^{4} \times[-\pi, \pi] .
$$

As we will see in Theorem 4.4, the region

$$
A_{(v, t)}=\left\{x \in S^{3}: d_{v}(x)<t\right\}
$$

has finite perimeter and so $\Sigma_{(v, t)} \in \mathcal{Z}_{2}\left(S^{3}\right)$ for all $(v, t)$ in $B^{4} \times[-\pi, \pi]$.

The diamater of $S^{3}$ is $\pi$ and thus $\Sigma_{(v, \pi)}=\Sigma_{(v,-\pi)}=0$ in $\mathcal{Z}_{2}\left(S^{3}\right)$.

4.3. Heintze-Karcher type-inequality. The next theorem is one of the reasons why the canonical family is interesting. A related result appears in Proposition 1 of [39].

4.4. Theorem. We have, for every $(v, t) \in B^{4} \times(-\pi, \pi)$,

$$
\text { area }\left(\Sigma_{(v, t)}\right) \leq \mathcal{W}(\Sigma)
$$

Moreover, if $\Sigma$ is not a geodesic sphere and

$$
\text { area }\left(\Sigma_{(v, t)}\right)=\mathcal{W}(\Sigma)
$$

then $t=0$ and $\Sigma_{v}$ is a minimal surface.

Proof. Let $N_{v}$ be the normal vector to $\Sigma_{v}=F_{v}(\Sigma)$ and consider the smooth map

$$
\psi_{(v, t)}: \Sigma_{v} \rightarrow S^{3}, \quad \psi_{(v, t)}(y)=\exp _{y}\left(t N_{v}(y)\right)=\cos t y+\sin t N_{v}(y) .
$$

Clearly $\Sigma_{(v, t)} \subset \psi_{(v, t)}\left(\Sigma_{v}\right)$ but in fact the following stronger inclusion holds

$$
\Sigma_{(v, t)} \subset \psi_{(v, t)}\left(\left\{p \in \Sigma_{v}: \operatorname{Jac} \psi_{(v, t)}(p) \geq 0\right\}\right)
$$

because if $q \in \Sigma_{(v, t)}$ and $\gamma$ is the minimizing geodesic connecting $\gamma(0) \in \Sigma_{v}$ to $\gamma(t)=q$, then $\gamma$ contains no focal points to $\Sigma_{v}$ (except possibly $q$ ). 
Claim: We have

$\operatorname{Jac} \psi_{(v, t)}(y)=\left(1+H(v)^{2}\right)-(\sin t+H(v) \cos t)^{2}-\frac{\left(k_{1}(v)-k_{2}(v)\right)^{2}}{4} \sin ^{2} t$, where $k_{1}(v)$ and $k_{2}(v)$ are the principal curvatures of $\Sigma_{v}$ at $y$ and $H(v)=$ $\frac{k_{1}(v)+k_{2}(v)}{2}$ is the mean curvature.

We proceed like in [39]: Let $\left\{e_{1}, e_{2}\right\} \subset T_{y} \Sigma_{v}$ be an orthonormal basis of principal directions with principal curvatures $k_{1}(v)$ and $k_{2}(v)$, respectively. Hence

$$
D \psi_{(v, t)} e_{y} e_{i}=\left(\cos t-k_{i}(v) \sin t\right) e_{i}
$$

from which we conclude that

$$
\operatorname{Jac} \psi_{(v, t)}(y)=\left(\cos t-k_{1}(v) \sin t\right)\left(\cos t-k_{2}(v) \sin t\right) .
$$

The claim follows by expanding this out.

Using the area formula, the above claim, and the conformal invariance of the Willmore energy we obtain

$$
\begin{aligned}
\operatorname{area}\left(\Sigma_{(v, t)}\right) & \leq \operatorname{area}\left(\psi_{(v, t)}\left(\left\{\operatorname{Jac} \psi_{(v, t)}(p) \geq 0\right\}\right)\right) \\
& \leq \int_{\left\{\operatorname{Jac} \psi_{(v, t)} \geq 0\right\}}\left(\operatorname{Jac} \psi_{(v, t)}\right) d \mu \\
& \leq \int_{\left\{\operatorname{Jac} \psi_{(v, t)}(p) \geq 0\right\}}\left(1+H(v)^{2}\right)-\sin ^{2} t \frac{\left(k_{1}(v)-k_{2}(v)\right)^{2}}{4} d \mu \\
& \leq \int_{\Sigma_{v}} 1+H(v)^{2} d \mu=\mathcal{W}\left(F_{v}(\Sigma)\right)=\mathcal{W}(\Sigma) .
\end{aligned}
$$

If equality holds for some $(v, t) \in B^{4} \times(-\pi, \pi)$, we obtain from the set of inequalities above that $\left\{\operatorname{Jac} \psi_{(v, t)} \geq 0\right\}=\Sigma$ and

$$
\frac{\sin ^{2} t}{2} \int_{\Sigma_{v}}|\AA|^{2} d \mu=\frac{\sin ^{2} t}{2} \int_{\Sigma}|\AA|^{2} d \mu=0,
$$

where $\AA$ denotes the trace-free part of the second fundamental form. This implies the rigidity statement.

4.5. Boundary behavior. It is important that to understand the behavior of $\Sigma_{(v, t)}$ as $(v, t)$ approaches $\partial\left(B^{4} \times[-\pi, \pi]\right)$. Before stating the main result, we discuss which behavior should one expect.

Denote by $A^{*}$ the "exterior" of $\Sigma=\partial A$, i.e., $A^{*}=S^{3} / \bar{A}$.

If $\left(v_{n}, t_{n}\right) \in B^{4} \times[-\pi, \pi]$ tends to $(v, t) \in A^{*} \times[-\pi, \pi]$, then one can see from (8) that $F_{v_{n}}(A)$ tends to $\{-v\}$. Thus $\Sigma_{\left(v_{n}, t_{n}\right)}$ is expected to converge to $\partial B_{t}(-v)$.

If $\left(v_{n}, t_{n}\right) \in B^{4} \times[-\pi, \pi]$ tends to $(v, t) \in A \times[-\pi, \pi]$, then one can see from (8) that $F_{v_{n}}(A)$ tends to $\bar{B}_{\pi}(v)=S^{3}$. Thus $\Sigma_{\left(v_{n}, t_{n}\right)}$ is expected to converge to $\partial B_{\pi+t}(v)$. 
The case where $\left(v_{n}, t_{n}\right) \in B^{4} \times[-\pi, \pi]$ tends to $(v, t) \in \Sigma \times[-\pi, \pi]$ is subtler, as we now explain.

Indeed, if $v \in \Sigma$, then $\Sigma_{t v}$ converges, as $t$ tends to 1 , to the unique great sphere tangent to $\Sigma$ at $v$. Thus the continuous function in $S^{3}$ given by $p \mapsto \mathbf{M}\left(\Sigma_{t p}\right)$ tends, as $t \rightarrow 1$, to a discontinuous function that is zero outside $\Sigma$ and $4 \pi$ along $\Sigma$. Hence, for any $0<\alpha<4 \pi$, there must exist a sequence $\left\{v_{i}\right\}_{i \in \mathbb{N}}$ in $B^{4}$ tending to $\Sigma$ so that $\mathbf{M}\left(\Sigma_{v_{i}}\right)$ tends to $\alpha$.

Therefore it is natural to expect that the behavior of $\Sigma_{\left(v_{n}, t_{n}\right)}$ depends on how $v_{n}$ approaches $v \in \Sigma$ and this is what we confirm in the next proposition.

Choose $N$ the normal vector to $\Sigma$ that points into $A^{*}$. For $p \in \Sigma$ and $k \in[-\infty,+\infty]$, set

$\bar{Q}_{p, k}=-\frac{k}{\sqrt{1+k^{2}}} p-\frac{1}{\sqrt{1+k^{2}}} N(p) \in S^{3} \quad$ and $\quad \bar{r}_{k}=\frac{\pi}{2}-\arctan k \in[0, \pi]$.

4.6. Proposition. Consider a sequence $\left(v_{n}, t_{n}\right) \in B^{4} \times[-\pi, \pi]$ converging to $(v, t) \in \bar{B}^{4} \times[-\pi, \pi]$.

(i) If $v \in B^{4}$ then

$$
\lim _{n \rightarrow \infty} \mathcal{F}\left(\Sigma_{\left(v_{n}, t_{n}\right)}-\Sigma_{(v, t)}\right)=0 .
$$

(ii) If $v \in A$ then

$$
\lim _{n \rightarrow \infty} \mathcal{F}\left(\Sigma_{\left(v_{n}, t_{n}\right)}-\partial B_{\pi+t}(v)\right)=0 .
$$

(iii) If $v \in A^{*}$ then

$$
\lim _{n \rightarrow \infty} \mathcal{F}\left(\Sigma_{\left(v_{n}, t_{n}\right)}-\partial B_{t}(-v)\right)=0 .
$$

(iv) If $v=p \in \Sigma$ and

$$
v_{n}=\left(1-s_{n 1}\right)\left(\cos \left(s_{n 2}\right) p_{n}+\sin \left(s_{n 2}\right) N\left(p_{n}\right)\right), \quad \lim _{n \rightarrow \infty} \frac{s_{n 2}}{s_{n 1}}=k \in[-\infty,+\infty],
$$

then

$$
\lim _{n \rightarrow \infty} \mathcal{F}\left(\Sigma_{\left(v_{n}, t_{n}\right)}-\partial B_{\bar{r}_{k}+t}\left(\bar{Q}_{p, k}\right)\right)=0
$$

4.7. Remark: In (iv), $\theta=\arctan k$ is the limit of the angle with which $v_{n}$ approaches $p_{n}$ and

$$
\bar{Q}_{p, k}=-\sin \theta p-\cos \theta N(p), \quad \bar{r}_{k}=\frac{\pi}{2}-\theta .
$$

Sketch of proof of Proposition 4.6. We assume for simplicity that $t_{n}=0$ for all $n$. The proof in the general case can be found in [31, Proposition 5.3].

The symmetric difference between two sets $B, C \subset S^{3}$ is denoted by $B \Delta C=B \backslash C \cup C \backslash B$.

If $v \in B^{4}$ then $F_{v_{n}}$ converges to $F_{v}$ and so Proposition 4.6 (i) follows. 
If $v \in A$, choose $r$ small so that $B_{r}(v) \subset A$. From (8) we see that $F_{v_{n}}\left(B_{r}(v)\right)$ is a geodesic ball that tends to $S^{3}=B_{\pi}(v)$ as $n$ tends to infinity. Thus, given any $\delta>0, B_{\pi-\delta}(v) \subset A_{\left(v_{n}, 0\right)}$ for all $n$ large and so

$$
A_{\left(v_{n}, 0\right)} \Delta B_{\pi}(v) \subset B_{\delta}(-v) .
$$

In particular, $\lim _{n \rightarrow \infty} \mathbf{M}\left(A_{\left(v_{n}, 0\right)} \Delta B_{\pi}(v)\right)=0$ and so, because

$$
\mathcal{F}\left(\Sigma_{\left(v_{n}, 0\right)}-\partial B_{\pi}(v)\right) \leq \mathbf{M}\left(A_{\left(v_{n}, 0\right)} \Delta B_{\pi}(v)\right),
$$

Proposition 4.6(ii) follows.

Proposition 4.6 (iii) follows in the same way.

To prove property (iv) we note that we can choose $r_{0}$ sufficiently small so that, for all $q \in \Sigma$, we can find a ball of radius $r_{0}$ contained in $A$ and tangential to $\Sigma$ at $q$, and another ball of radius $r_{0}$ contained in the exterior of $A$, that we denoted by $A^{*}$, and tangential to $\Sigma$ at $q$. These balls are given, respectively, by

$$
\begin{aligned}
& B_{r_{0}}\left(\left(\cos r_{0}\right) q-\left(\sin r_{0}\right) N(q)\right) \subset A, \\
& \text { and } B_{r_{0}}\left(\left(\cos r_{0}\right) q+\left(\sin r_{0}\right) N(q)\right) \subset A^{*} .
\end{aligned}
$$

For any $q \in \Sigma$, consider $B_{q}=B_{\pi / 2}(-N(q))$, whose boundary is a great sphere tangent to $\Sigma$ at $q$.

We have for all $q \in \Sigma$ that

$$
\begin{aligned}
& B_{r_{0}}\left(\left(\cos r_{0}\right) q-\left(\sin r_{0}\right) N(q)\right) \subset B_{q}, \\
& B_{r_{0}}\left(\left(\cos r_{0}\right) q+\left(\sin r_{0}\right) N(q)\right) \subset S^{3} \backslash B_{q}
\end{aligned}
$$

and so it follows from (9) that

$$
\begin{aligned}
A \Delta B_{p_{n}} \subset S^{3} \backslash\left(B _ { r _ { 0 } } \left(\left(\cos r_{0}\right) p_{n}+\right.\right. & \left.\left(\sin r_{0}\right) N\left(p_{n}\right)\right) \\
& \left.\cup B_{r_{0}}\left(\left(\cos r_{0}\right) p_{n}-\left(\sin r_{0}\right) N\left(p_{n}\right)\right)\right) .
\end{aligned}
$$

Denoting the set on the right hand side by $\Delta\left(p_{n}, N\left(p_{n}\right), r_{0}\right)$ we have from the inclusion above that

$$
\begin{aligned}
F_{v_{n}}\left(B_{p_{n}}\right) \backslash F_{v_{n}}\left(\Delta\left(p_{n}, N\left(p_{n}\right), r_{0}\right)\right) \subset & F_{v_{n}}(A) \\
& \subset F_{v_{n}}\left(B_{p_{n}}\right) \cup F_{v_{n}}\left(\Delta\left(p_{n}, N\left(p_{n}\right), r_{0}\right)\right) .
\end{aligned}
$$

In Proposition B.1. of [31, Appendix B], after some long computations, we showed that given $\delta>0$ we have, for all $n$ sufficiently large,

$$
B_{\bar{r}_{k_{n}}-\delta}\left(\bar{Q}_{p_{n}, k_{n}}\right) \subset F_{v_{n}}\left(B_{p_{n}}\right) \backslash F_{v_{n}}\left(\Delta\left(p_{n}, N\left(p_{n}\right), r_{0}\right)\right) \subset F_{v_{n}}(A)
$$

and

$$
F_{v_{n}}\left(B_{p_{n}}\right) \cup F_{v_{n}}\left(\Delta\left(p_{n}, N\left(p_{n}\right), r_{0}\right)\right) \subset B_{\bar{r}_{k_{n}}+\delta}\left(\bar{Q}_{p_{n}, k_{n}}\right),
$$

where $k_{n}=s_{n 1} / s_{n 2}$. This implies that

$$
B_{\bar{r}_{k_{n}}-\delta}\left(\bar{Q}_{p_{n}, k_{n}}\right) \subset F_{v_{n}}(A) \subset B_{\bar{r}_{k_{n}}+\delta}\left(\bar{Q}_{p_{n}, k_{n}}\right) .
$$


Because $\bar{r}_{k_{n}}$ and $\bar{Q}_{p_{n}, k_{n}}$ converge to $\bar{r}_{k}$ and $\bar{Q}_{p, k}$, respectively, we obtain from the inclusion above and the arbitrariness of $\delta$ that

$$
\lim _{n \rightarrow \infty} \mathbf{M}\left(F_{v_{n}}(A) \Delta B_{\bar{r}_{k}}\left(\bar{Q}_{p, k}\right)\right)=0
$$

and so, because

$$
\mathcal{F}\left(\Sigma_{\left(v_{n}, 0\right)}-\partial B_{\bar{r}_{k}}\left(\bar{Q}_{p, k}\right)\right) \leq \mathbf{M}\left(F_{v_{n}}(A) \Delta B_{\bar{r}_{k}}\left(\bar{Q}_{p, k}\right)\right),
$$

we obtain Proposition 4.6 (iv).

4.8. Boundary blow-up. We use Proposition 4.6 to extend the canonical family defined in Section 4.2 to a continuous map $C$ from $\bar{B}^{4} \times[-\pi, \pi]$ to $\mathcal{Z}_{2}\left(S^{3}\right)$. Later, we reparametrize the map $C$ to obtain a map $\Phi$ that satisfies Theorem 4.1.

For any $s=\left(s_{1}, s_{2}\right) \in \mathbb{R}^{2}$ and $p \in \Sigma$, set

$$
\Lambda(p, s)=\left(1-s_{1}\right)\left(\cos \left(s_{2}\right) p+\sin \left(s_{2}\right) N(p)\right) .
$$

With $\varepsilon$ chosen sufficiently small and $r \leq 3 \varepsilon$, let $\Omega_{r}$ be a tubular neighborhood of radius $r$ around $\Sigma$ in $\bar{B}^{4}$ :

$$
\Omega_{r}=\left\{\Lambda(p, s):\left|\left(s_{1}, s_{2}\right)\right|<r, s_{1} \geq 0, p \in \Sigma\right\} .
$$

Consider the continuous map $T: \bar{B}^{4} \rightarrow \bar{B}^{4}$ such that

- $T$ is the identity on $\bar{B}^{4} \backslash \Omega_{3 \varepsilon}$;

- on $\Omega_{3 \varepsilon}$ we have

$$
T(\Lambda(p, s))=\Lambda(p, \phi(|s|) s)
$$

where $\phi$ is smooth, zero on $[0, \varepsilon]$, strictly increasing on $[\varepsilon, 2 \varepsilon]$, and one on $[2 \varepsilon, 3 \varepsilon]$.

Note that $T$ maps $B^{4} \backslash \bar{\Omega}_{\varepsilon}$ homeomorphically onto $B^{4}$ and $T$ maps $\bar{\Omega}_{\varepsilon}$ onto $\Sigma$ by nearest point projection

Recall the expressions for $\bar{Q}_{p, k}$ and $\bar{r}_{k}$ in Section 4.5 and consider the functions $\bar{Q}: \bar{\Omega}_{\varepsilon} \rightarrow S^{3}$ such that

$$
\bar{Q}\left(\Lambda\left(p,\left(s_{1}, s_{2}\right)\right)\right)=\bar{Q}_{p, k} \quad \text { where } k=\frac{s_{2}}{\sqrt{\varepsilon^{2}-s_{2}^{2}}},
$$

and $\bar{r}: \bar{\Omega}_{\varepsilon} \rightarrow[0, \pi]$ such that

$$
\bar{r}\left(\Lambda\left(p,\left(s_{1}, s_{2}\right)\right)=\bar{r}_{k} \quad \text { where } k=\frac{s_{2}}{\sqrt{\varepsilon^{2}-s_{2}^{2}}} .\right.
$$

Note that $\bar{Q}$ and $\bar{r}$ are constant along the radial directions and the reason why we considered these functions is because of the following theorem. 
4.9. Theorem. The map below is well defined and continuous in the flat topology:

$$
C(v, t)=\left\{\begin{aligned}
C: \bar{B}^{4} \times[-\pi, \pi] & \rightarrow \mathcal{Z}_{2}\left(S^{3}\right), \\
\Sigma_{(T(v), t)} & \text { if } v \in B^{4} \backslash \bar{\Omega}_{\varepsilon}, \\
\partial B_{\pi+t}(T(v)) & \text { if } v \in A \backslash \bar{\Omega}_{\varepsilon}, \\
\partial B_{t}(-T(v)) & \text { if } v \in A^{*} \backslash \bar{\Omega}_{\varepsilon}, \\
\partial B_{\bar{r}(v)+t}(\bar{Q}(v)) & \text { if } v \in \bar{\Omega}_{\varepsilon} .
\end{aligned}\right.
$$

Furthermore,

$$
\mathbf{M}(C(v, t)) \leq \mathcal{W}(\Sigma) \quad \text { for all } \quad(v, t) \in \bar{B}^{4} \times[-\pi, \pi]
$$

SKETCH OF PROOF. We go through some of the main computations and leave the remaining details for the reader to see in [31, Theorem 5.1].

The functions $\bar{Q}$ and $\bar{r}$ are continuous and so $C$ is continuous on $\bar{\Omega}_{\varepsilon} \times$ $[-\pi, \pi]$.

The map $T$ sends $B^{4} \backslash \bar{\Omega}_{\varepsilon}$ homeomorphically onto $B^{4}$ and so we have from Proposition 4.6 (i) that $C$ is continuous on $B^{4} \backslash \bar{\Omega}_{\varepsilon} \times[-\pi, \pi]$.

Suppose now that $\left(v_{n}, t_{n}\right)$ in $B^{4} \backslash \bar{\Omega}_{\varepsilon}$ tends to $(v, t)$ with $v \in A \backslash \bar{\Omega}_{\varepsilon}$. From the properties defining $T$ we have that $T\left(v_{n}\right)$ tends to $T(v) \in A$ and so we obtain from Proposition 4.6 (i) that, in the flat topology,

$$
\lim _{n \rightarrow \infty} C\left(v_{n}, t_{n}\right)=\lim _{n \rightarrow \infty} \Sigma_{\left(T\left(v_{n}\right), t_{n}\right)}=\partial B_{\pi+t}(T(v))=C(v, t) .
$$

A similar reasoning shows that if $\left(v_{n}, t_{n}\right)$ in $B^{4} \backslash \bar{\Omega}_{\varepsilon}$ tends to $(v, t)$ with $v \in A^{*} \backslash \bar{\Omega}_{\varepsilon}$, then $C\left(v_{n}, t_{n}\right)$ tends to $C(v, t)$. Thus $C$ is continuous on $\bar{B}^{4} \backslash$ $\bar{\Omega}_{\varepsilon} \times[-\pi, \pi]$.

The last case we consider is when $\left(v_{n}, t_{n}\right)$ tends to $(v, t)$ with $v_{n} \in B^{4} \backslash \bar{\Omega}_{\varepsilon}$ and $v \in B^{4} \cap \partial \bar{\Omega}_{\varepsilon}$. The remaining cases can be seen in [31, Theorem 5.1]. We have

$$
v=\Lambda\left(p,\left(\sqrt{\varepsilon^{2}-s_{2}^{2}}, s_{2}\right)\right) \text { for some }\left|s_{2}\right|<\varepsilon, p \in \Sigma,
$$

and $v_{n}=\Lambda\left(p_{n}, s_{n}\right)$ so that

$$
\lim _{n \rightarrow \infty} p_{n}=p \quad \text { and } \quad \lim _{n \rightarrow \infty} s_{n}=\lim _{n \rightarrow \infty}\left(s_{n 1}, s_{n 2}\right)=\left(\sqrt{\varepsilon^{2}-s_{2}^{2}}, s_{2}\right) \text {. }
$$

From (10) we have $T\left(v_{n}\right)=\Lambda\left(p_{n}, \phi\left(\left|s_{n}\right|\right) s_{n}\right)$ and thus we obtain from Proposition 4.6 (iv) that, in the flat topology,

$$
\lim _{n \rightarrow \infty} C\left(v_{n}, t_{n}\right)=\lim _{n \rightarrow \infty} \Sigma_{\left(T\left(v_{n}\right), t_{n}\right)}=\partial B_{\bar{r}_{k}+t}\left(\bar{Q}_{k}\right)
$$

where

$$
k=\lim _{n \rightarrow \infty} \frac{s_{n 2}}{s_{n 1}}=\frac{s_{2}}{\sqrt{\varepsilon^{2}-s_{2}^{2}}} .
$$


Because

$$
C(v, t)=\partial B_{\bar{r}_{k}+t}\left(\bar{Q}_{k}\right), \quad \text { where } k=\frac{s_{2}}{\sqrt{\varepsilon^{2}-s_{2}^{2}}},
$$

we have that $C\left(v_{n}, t_{n}\right)$ tends to $C(v, t)$ in the flat topology.

Finally, if $(v, t) \in \bar{B}^{4} \backslash \Omega_{\varepsilon}$, we have from Theorem 4.4 that $\mathbf{M}(C(v, t)) \leq$ $\mathcal{W}(\Sigma)$. If $(v, t) \in S^{3} \cup \Omega_{\varepsilon}$, then $C(v, t)$ is a round sphere and so $\mathbf{M}(C(v, t) \leq$ $4 \pi \leq \mathcal{W}(\Sigma)$ because every compact surface has Willmore energy no smaller than $4 \pi$.

4.10. Extended Gauss map. From Theorem 4.9 we see that for every $(v, t) \in S^{3}, C(v, t)$ is a geodesic sphere centered at $\bar{Q}(v)$ given by (with an obvious abuse of notation)

$$
\bar{Q}: S^{3} \rightarrow S^{3}, \quad \bar{Q}(v)=\left\{\begin{aligned}
-T(v) & \text { if } v \in A^{*} \backslash \bar{\Omega}_{\varepsilon}, \\
T(v) & \text { if } v \in A \backslash \bar{\Omega}_{\varepsilon}, \\
\bar{Q}(v) & \text { if } v \in S^{3} \cap \bar{\Omega}_{\varepsilon},
\end{aligned}\right.
$$

where, with $v=\Lambda\left(p,\left(0, s_{2}\right)\right) \in S^{3} \cap \bar{\Omega}_{\varepsilon}$, recall that

$$
\bar{Q}(v)=-\frac{k}{\sqrt{1+k^{2}}} p-\frac{1}{\sqrt{1+k^{2}}} N(p) \quad \text { where } k=\frac{s_{2}}{\sqrt{\varepsilon^{2}-s_{2}^{2}}} .
$$

In particular, note that $\bar{Q}(\Lambda(p,(0,0))=-N(p)$ is simply the Gauss map.

4.11. Theorem. The map $\bar{Q}: S^{3} \rightarrow S^{3}$ has degree $g$, the genus of $\Sigma$.

Proof. We will use the fact that $\bar{Q}$ is piecewise smooth. Let $d V$ denote the volume form of $S^{3}$ and $\nabla$ the induced connection on $S^{3}$.

Since $\bar{Q}=-T$ on $A^{*} \backslash \bar{\Omega}_{\varepsilon}$, we have from the definition of $T$ that $\bar{Q}$ is an orientation-preserving diffeomorphism of $A^{*} \backslash \Omega_{\varepsilon}$ onto $-\overline{A^{*}}$. Therefore

$$
\int_{A^{*} \mid \bar{\Omega}_{\varepsilon}} \bar{Q}^{*}(d V)=\int_{-A^{*}} d V=\operatorname{vol}\left(A^{*}\right)
$$

Since $\bar{Q}=T$ on $A \backslash \bar{\Omega}_{\varepsilon}$, we have from the definition of $T$ that $\bar{Q}$ is an orientation-preserving diffeomorphism of $A \backslash \bar{\Omega}_{\varepsilon}$ onto $\bar{A}$. Therefore

$$
\int_{A \backslash \bar{\Omega}_{\varepsilon}} \bar{Q}^{*}(d V)=\int_{A} d V=\operatorname{vol}(A) .
$$

Recall that $\left\{e_{1}, e_{2}, e_{3}\right\} \in T_{p} S^{3}$ is a positive basis if $\left\{e_{1}, e_{2}, e_{3}, p\right\}$ is a positive basis of $\mathbb{R}^{4}$, and $\left\{e_{1}, e_{2}\right\} \in T_{p} \Sigma$ is a positive basis if $\left\{e_{1}, e_{2}, N(p)\right\}$ is a positive basis of $T_{p} S^{3}$.

Consider the diffeomorphism $G: \Sigma \times[-\pi / 2, \pi / 2] \rightarrow S^{3} \cap \bar{\Omega}_{\varepsilon}$ defined by

$$
G(p, \theta)=\Lambda(p,(0, \varepsilon \sin \theta))=\cos (\varepsilon \sin \theta) p+\sin (\varepsilon \sin \theta) N(p) .
$$

The orientation of $\Sigma \times[-\pi / 2, \pi / 2]$ is chosen so that $\left\{e_{1}, e_{2}, \partial_{\theta}\right\}$ is a positive basis whenever $\left\{e_{1}, e_{2}\right\}$ is a positive basis of $\Sigma$. We have

$$
G_{*}\left(e_{1} \wedge e_{2} \wedge \partial_{\theta}\right)_{\mid(p, 0)}=\varepsilon e_{1} \wedge e_{2} \wedge N(p)
$$

and thus $G$ is orientation preserving. 
Consider $Q=\bar{Q} \circ G: \Sigma \times[-\pi / 2, \pi / 2] \rightarrow S^{3}$ given by

$$
Q(p, \theta)=-\sin \theta p-\cos \theta N(p) .
$$

Hence

$$
\int_{S^{3} \cap \bar{\Omega}_{\varepsilon}} \bar{Q}^{*}(d V)=\int_{\Sigma \times[-\pi / 2, \pi / 2]} G^{*}\left(\bar{Q}^{*}(d V)\right)=\int_{\Sigma \times[-\pi / 2, \pi / 2]} Q^{*}(d V) .
$$

Let $\left\{e_{1}, e_{2}\right\}$ be a positive orthonormal basis of $T_{p} \Sigma$ which diagonalizes the second fundamental form:

$$
\nabla_{e_{i}} N=-k_{i} e_{i} \quad \text { for } i=1,2
$$

We have

$$
D Q_{\mid(p, \theta)}\left(\partial_{\theta}\right)=-\cos \theta p+\sin \theta N(p),
$$

and

$$
D Q_{\mid(p, \theta)}\left(e_{i}\right)=\left(-\sin \theta+\cos \theta k_{i}\right) e_{i} \text { for } i=1,2,
$$

and thus, denoting by $\operatorname{vol}_{\mathbb{R}^{4}}$ the standard volume form of $\mathbb{R}^{4}$, we have

$$
\begin{aligned}
Q^{*}(d V)_{\mid(p, \theta)}\left(e_{1}, e_{2}, \partial_{\theta}\right) & =d V_{\mid Q(p, \theta)}\left(D Q\left(e_{1}\right), D Q\left(e_{2}\right), D Q\left(\partial_{\theta}\right)\right) \\
& =\operatorname{vol}_{\mathbb{R}^{4} \mid Q(p, \theta)}\left(D Q\left(e_{1}\right), D Q\left(e_{2}\right), D Q\left(\partial_{\theta}\right), Q(p, \theta)\right) \\
& =\left(-\sin \theta+\cos \theta k_{1}\right)\left(-\sin \theta+\cos \theta k_{2}\right)(-1)
\end{aligned}
$$

since

$$
\begin{aligned}
& D Q_{\mid(p, \theta)}\left(\partial_{\theta}\right) \wedge Q(p, \theta)= \\
& \quad(-\cos \theta p+\sin \theta N(p)) \wedge(-\sin \theta p-\cos \theta N(p))=-N(p) \wedge p .
\end{aligned}
$$

The Gauss equation implies that $K=1+k_{1} k_{2}$, where $K$ denotes the Gauss curvature of $\Sigma$ and so we conclude that

$$
\begin{gathered}
\int_{\Sigma \times[-\pi / 2, \pi / 2]} Q^{*}(d V) \\
=-\int_{\Sigma} \int_{-\pi / 2}^{\pi / 2}\left(k_{1} k_{2} \cos ^{2} \theta-\left(k_{1}+k_{2}\right) \sin \theta \cos \theta+\sin ^{2} \theta\right) d t d \mu \\
=-\frac{\pi}{2} \int_{\Sigma}(K-1) d \mu-\frac{\pi}{2} \int_{\Sigma} d \mu=-\pi^{2} \chi(\Sigma)=\pi^{2}(2 g-2) .
\end{gathered}
$$

In the calculation above we have used that

- $\int_{-\pi / 2}^{\pi / 2} \cos ^{2} \theta d \theta=\int_{-\pi / 2}^{\pi / 2} \sin ^{2} \theta d \theta=\frac{\pi}{2}$,

- $\int_{-\pi / 2}^{\pi / 2} \sin \theta \cos \theta d \theta=0$, 
Finally, since $\operatorname{vol}\left(S^{3}\right)=2 \pi^{2}$, we combine (12), (13), and (14) to obtain

$$
\begin{aligned}
& \int_{S^{3}} \bar{Q}^{*}(d V)= \int_{A^{*} \backslash \bar{\Omega}_{\varepsilon}} \bar{Q}^{*}(d V)+\int_{A \backslash \bar{\Omega}_{\varepsilon}} \bar{Q}^{*}(d V)+\int_{S^{3} \cap \bar{\Omega}_{\varepsilon}} \bar{Q}^{*}(d V) \\
&=\operatorname{vol}\left(A^{*}\right)+\operatorname{vol}(A)+\int_{\Sigma \times[-\pi / 2, \pi / 2]} Q^{*}(d V) \\
&=2 \pi^{2}+\pi^{2}(2 g-2)=2 \pi^{2} g=g \int_{S^{3}} d V .
\end{aligned}
$$

It follows that $\operatorname{deg}(Q)=g$.

4.12. Sketch of proof of Theorem 4.1. The map $\Phi$ will be obtained by composing the map $C$ given by Theorem 4.9 with a suitable homomorphism from $I^{5}$ to $\bar{B}^{4} \times[-\pi, \pi]$.

From the expression defining the map $C$ we have at once that $C\left(\bar{B}^{4}, \pi\right)=$ $C\left(\bar{B}^{4},-\pi\right)=0$ because the range of the function $\bar{r}$ is $[0, \pi]$ and, as we remarked at the end of Section $4.2, \Sigma_{v, \pi}=\Sigma_{v,-\pi}=0$ for all $v \in B^{4}$.

Hence we can extend $C$ to $\bar{B}^{4} \times \mathbb{R}$ by defining

$$
C=0 \quad \text { on } \quad \bar{B}^{4} \times(\mathbb{R} \backslash[-\pi, \pi]) .
$$

We also extend $\bar{r}$ to be defined on $\bar{B}^{4}$ in the following way. First set $\bar{r}$ to be $\pi$ on $A \backslash \Omega_{\varepsilon}$ and 0 on $A \backslash \Omega_{\varepsilon}$. This way we obtain, by Theorem 4.9, a continuous function on $S^{3} \cup \bar{\Omega}_{\varepsilon}$ that we can extend, in any chosen way, to a continuous function $\bar{r}: \bar{B}^{4} \rightarrow[0, \pi]$.

We now have

$$
C(v, t)=\partial B_{\bar{r}(v)+t}(\bar{Q}(v)) \quad \text { for all }(v, t) \in S^{3} \times \mathbb{R} .
$$

Choose an orientation preserving homeomorphism $f: I^{4} \rightarrow \bar{B}^{4}$ (hence $f_{\mid \partial I^{4}}$ is a homeomorphism from $\partial I^{4}$ onto $\left.S^{3}\right)$ and consider

$$
\gamma: \mathbb{R} \rightarrow \mathbb{R}, \quad \gamma(s)=0 \text { if } s \leq \frac{1}{2}, \quad \gamma(s)=2 s-1 \text { if } s \geq \frac{1}{2} .
$$

The map $\Phi: I^{5} \rightarrow \mathcal{Z}_{2}\left(S^{3}\right)$ is given by

$$
\Phi(x, t)=C\left(f(x), 2 \pi(2 t-1)+\gamma(|f(x)|)\left(\frac{\pi}{2}-\bar{r}(f(x))\right)\right) .
$$

Theorem 4.1 (1) and (2) follow from (15). Theorem 4.1 (3) follows from Theorem 4.9 and Theorem 4.1 (4) follows from Theorem 4.11 because $Q=$ $\bar{Q} \circ f$ and the degree is invariant by composition with homeomorphisms.

To show the map $\Phi$ has no concentration of mass we refer the reader to [31, Section 13] because the proof is rather lengthy due to the fact that one needs to examine carefully how the conformal maps $F_{v}$ deform the surface $\Sigma$ as $|v|$ tends to one. 


\section{5. $2 \pi^{2}$-Theorem}

In this section we combine Theorem 3.1 and Theorem 4.1 with a characterization, found by Urbano, of the Clifford torus in terms of its index in order to show that the Clifford torus is the minimal surface of least area after a great sphere. As a corollary we deduce an improvement of Theorem 3.1 that we call the $2 \pi^{2}$-Theorem.

5.1. Urbano's Theorem. Let $\Sigma$ be a closed embedded minimal surface in $S^{3}$. Its Jacobi operator is defined to be

$$
L f=\Delta f+\left(|A|^{2}+2\right) f, \quad f \in C^{\infty}(\Sigma) .
$$

The Jacobi operator is the second variation for the area functional. More precisely, if $N$ is the unit normal vector to $\Sigma$, then for every $f \in C^{\infty}(\Sigma)$ we have

$$
\frac{d^{2}}{(d t)^{2}} \underset{\mid t=0}{\operatorname{area}}\left(P_{t}(\Sigma)\right)=-\int_{\Sigma} f L f d \mu,
$$

where $\left\{P_{t}\right\}_{-\varepsilon<t<\varepsilon}$ is a one parameter family of diffeomorphisms generated by the normal vector field $X=f N$.

The index of $\Sigma$, denoted by index $(\Sigma)$, is defined to be the number of negative eigenvalues of $-L$.

Urbano proved in $[44]$ the following elegant characterization of the Clifford torus.

5.2. Theorem. Assume $\Sigma$ is a closed embedded minimal surface in $S^{3}$ having index $(\Sigma) \leq 5$.

Then $\Sigma$ is either a great sphere or the Clifford torus up to ambient isometries

Sketch OF PROOF: Let $f_{0}$ be the lowest eigenfunction for $L$ and set

$$
f_{i}(x)=\left\langle e_{i}, N(x)\right\rangle, \quad i=1, \ldots, 4,
$$

where $e_{i} \in \mathbb{R}^{4}$ is a coordinate vector and $N$ is a unit normal vector to $\Sigma$.

Denote by $V$ the vector subspace of $C^{\infty}(\Sigma)$ spanned by $f_{1}, \ldots, f_{4}$.

Claim: If $\operatorname{dim} V \leq 3$, then $\Sigma$ is a great sphere.

In this case we can find a vector $0 \neq w \in \mathbb{R}^{4}$ so that $\langle w, N(x)\rangle=0$ for all $x \in \Sigma$. Let $h: S^{3} \rightarrow \mathbb{R}$ be given by $h(x)=\langle x, w\rangle$. The way $w$ was chosen implies that the conformal vector field $U(x)=\nabla h(x)$ of $S^{3}$ satisfies $U(x) \in T_{x} \Sigma$ for all $x \in \Sigma$. This means $\Sigma$ is invariant by the flow generated by $U$, but this is only possible if $\Sigma$ is totally geodesic.

A simple computation shows that $\Delta f_{i}=-|A|^{2} f_{i}$ and so $L\left(f_{i}\right)=2 f_{i}$ for all $i=1, \ldots, 4$ and thus $V$ is a negative eigenspace. The lowest eigenvalue always has multiplicity one and so if $\operatorname{index}(\Sigma) \leq 4$, then $\operatorname{dim} V \leq 3$, in which case the claim above implies $\Sigma$ is a great sphere with index one. 
Assume now $\Sigma$ has index 5. From [27] we can find a conformal map $F_{v}$ of $S^{3}$ so that

$$
\int_{\Sigma}\left\langle F_{v}(x), e_{i}\right\rangle f_{0}(x) d \mu=0 \quad \text { for all } i=1, \ldots 4 .
$$

Hence, because the second eigenvalue is -2 , we have

(16) $-\int_{\Sigma} L\left(\left\langle F_{v}(x), e_{i}\right\rangle\right)\left\langle F_{v}(x), e_{i}\right\rangle d \mu \geq-2 \int_{\Sigma}\left\langle F_{v}(x), e_{i}\right\rangle^{2} d \mu, \quad i=1, \ldots 4$

and so, after integrating by parts and adding,

$$
\sum_{i=1}^{4} \int_{\Sigma}\left|\nabla\left\langle F_{v}(x), e_{i}\right\rangle\right|^{2} d \mu \geq \sum_{i=1}^{4} \int_{\Sigma}|A|^{2}\left\langle F_{v}(x), e_{i}\right\rangle^{2} d \mu=\int_{\Sigma}|A|^{2} d \mu .
$$

The left hand side is 2 area $\left(F_{v}(\Sigma)\right)$ and thus, using Gauss Bonnet, the inequality above becomes

$$
2 \text { area }\left(F_{v}(\Sigma)\right) \geq 2 \text { area }(\Sigma)-8 \pi(1-g) .
$$

From formula (1.12) of [34] we have that

$$
\operatorname{area}\left(F_{v}(\Sigma)\right)=\operatorname{area}(\Sigma)-4 \int_{\Sigma} \frac{\langle v, N(x)\rangle^{2}}{|x-v|^{4}} d \mu
$$

and this implies

$$
2 \pi(1-g) \geq \int_{\Sigma} \frac{\langle v, N(x)\rangle^{2}}{|x-v|^{4}} d \mu .
$$

This inequality forces the genus $g$ to be either 0 or 1 . If $g=0$, then Almgren [4] showed that $\Sigma$ is a great sphere and thus cannot have index 5. Hence $g=1, v$ must be zero, and the inequalities derived above become equalities. In particular, equality holds in (16) with $v=0$ and this implies that the coordinate functions satisfy $L x_{i}=-2 x_{i}$ for all $i=1, \ldots, 4$. Because the coordinate functions are harmonic on $\Sigma$ this means that $|A|^{2}=2$. A classical result [10] states that $\Sigma$ has to be a Clifford torus up to ambient isometries.

Theorem 3.1 and Theorem 4.1 allows us to go from a local characterization of the Clifford torus in terms of its index to a global characterization in terms of its area.

5.3. Theorem. Let $\Sigma \subset S^{3}$ be a closed embedded minimal surface of genus $g \geq 1$.

Then area $(\Sigma) \geq 2 \pi^{2}$, and area $(\Sigma)=2 \pi^{2}$ if and only if $\Sigma$ is the Clifford torus up to isometries of $S^{3}$. 
5.4. Sketch of proof of Theorem 5.3. Choose $\Sigma$ to be the minimal surface with least area among all minimal surfaces in $S^{3}$ with genus greater than or equal to 1 . (The existence of $\Sigma$ follows from standard arguments in Geometric Measure Theory. This is explained in Appendix A of [31].)

It suffices to show that $\Sigma$ is the Clifford torus up to isometries of $S^{3}$.

Consider the family $\Phi: I^{5} \rightarrow \mathcal{Z}_{2}\left(S^{3}\right)$ associated to $\Sigma$ that is given by Theorem 4.1. Set $\Phi_{0}=\Phi_{\mid \partial I^{5}}$ and consider the relative homotopy class $[\Phi]$ defined in Section 2.11.

5.5. Lemma. $\mathbf{L}([\Phi])=\sup _{x \in I^{5}} \mathbf{M}(\Phi(x))=\operatorname{area}(\Sigma)$.

Proof. From the fact that $\Sigma$ is a minimal surface we obtain at once from Theorem 4.1 (3) that

$$
\mathbf{L}([\Phi]) \leq \sup _{x \in I^{5}} \mathbf{M}(\Phi(x)) \leq \operatorname{area}(\Sigma) .
$$

It suffices to show that $\mathbf{L}([\Phi]) \geq \operatorname{area}(\Sigma)$.

Suppose there is $\Psi \in[\Phi]$ so that $\sup _{x \in I^{5}} \mathbf{M}(\Psi(x))<\operatorname{area}(\Sigma)$. Using Theorem 2.13 we obtain $\Pi_{\mathbf{M}}$ a $\left(I^{5}, \mathbf{M}\right)$-homotopy class of mappings into $\left(\mathcal{Z}_{2}\left(S^{3} ; \mathbf{M}\right), \Phi_{0}\right)$ such that

$$
\mathbf{L}([\Phi]) \leq \mathbf{L}\left(\Pi_{\mathbf{M}}\right) \leq \sup \left\{\mathbf{M}(\Psi(x)): x \in I^{5}\right\}<\operatorname{area}(\Sigma) .
$$

Because the genus of $\Sigma$ is positive, we obtain from Theorem 4.1 that all conditions of Theorem 3.1 are satisfied and hence $\mathbf{L}\left(\Pi_{\mathbf{M}}\right) \geq \mathbf{L}([\Phi])>4 \pi$. Thus one can use Theorem 2.12 to conclude the existence of a minimal surface $S$ (with possible multiplicities) so that

$$
4 \pi<\mathbf{L}([\Phi]) \leq \mathbf{M}(S)=\mathbf{L}\left(\Pi_{\mathbf{M}}\right)<\operatorname{area}(\Sigma) \leq 2 \pi^{2},
$$

where the last inequality follows from the fact that $\Sigma$ must necessarily have area smaller or equal than the area of the Clifford torus.

From the inequality above we deduce that $S$ has to be a multiplicity one minimal surface because otherwise its area would be bigger or equal than $8 \pi$ and that $S$ cannot have genus zero because then it would be a great sphere [4] with area $4 \pi$. Hence, from the way $\Sigma$ was selected, we have $\operatorname{area}(S) \geq \operatorname{area}(\Sigma)$ and this contradicts (18).

The goal now is to show that $\Sigma$ has index $(\Sigma) \leq 5$. If true, the theorem follows because we obtain from Theorem 5.2 that $\Sigma$ is either a great sphere or a Clifford torus. Only the latter case can happen because the genus of $\Sigma$ is positive and so $\Sigma$ is the Clifford torus up to isometries of $S^{3}$.

Assume index $(\Sigma) \geq 6$. The idea is to slightly perturb the 5-dimensional canonical family associated with $\Sigma$ defined in Section 4.2 in order to produce a map $\Phi^{\prime}: I^{5} \rightarrow \mathcal{Z}_{2}\left(S^{3}\right)$ in $[\Phi]$ so that

$$
\mathbf{L}([\Phi]) \leq \sup _{x \in I^{5}} \mathbf{M}\left(\Phi^{\prime}(x)\right)<\mathbf{M}(\Sigma)=\mathbf{L}([\Phi]) .
$$

This is a contradiction. 
We now give some details of the construction. In what follows $N_{v}$ is the unit normal vector to $F_{v}(\Sigma)$ with the same direction as $D F_{v}(N)$. Choose $\delta>0$ small such that the map

$$
P: B_{\delta}^{4}(0) \times(-\delta, \delta) \times \Sigma \rightarrow S^{3}, \quad P_{(v, t)}(x)=(\cos t) F_{v}(x)+(\sin t) N_{v}(x)
$$

has $\Sigma_{(v, t)}=P_{(v, t)}(\Sigma)$ and $P_{(v, t)}$ is an embedding of $\Sigma$ into $S^{3}$.

5.6. Lemma. The function $f: B^{4} \times[-\pi, \pi] \rightarrow[0, \infty], f(v, t)=\mathbf{M}\left(\Sigma_{v, t}\right)$ has a unique global non-degenerate maximum at the origin.

Proof. From Lemma 4.4 we have that $f(v, t) \leq \mathcal{W}(\Sigma)=f(0,0)$ for all $(v, t)$ in $B^{4} \times[-\pi, \pi]$. Suppose equality holds for some $(v, t)$.

Because $\Sigma$ is not a round sphere we obtain from Lemma 4.4 that $\Sigma_{v}$ is a minimal surface with the same area as $\Sigma$. Thus, identity (17) implies that $h(x)=\langle N(x), v\rangle$ vanishes on $\Sigma$ and we already saw that implies $\Sigma$ to be a round sphere (see proof of Theorem 5.2). This is a contradiction and so

$$
f(v, t)<f(0,0) \quad \text { fort all }(v, t) \in B^{4} \times[-\pi, \pi] \backslash\{(0,0)\} .
$$

It remains to see that $f$ has a non-degenerate maximum at the origin.

Let $\left\{e_{1}, e_{2}, e_{3}, e_{4}\right\}$ be the standard orthonormal basis of $\mathbb{R}^{4}$. For $x \in \Sigma$, define $\psi_{i}(x)=\left\langle N(x), e_{i}\right\rangle$ for each $1 \leq i \leq 4$, and $\psi_{5}(x)=1$. Denote by $E$ the subspace of $C^{\infty}(\Sigma)$ spanned by $\left\{\psi_{i}\right\}_{1 \leq i \leq 5}$. Notice that $L \psi_{i}=2 \psi_{i}$ for $1 \leq i \leq 4$ (see $[\mathbf{4 4}]$ ), a fact which was already observed in the proof of Theorem 5.2.

If $1 \leq i \leq 4, x \in \Sigma$, we have

$$
\left\langle\frac{d}{d s}{ }_{\mid s=0} P_{\left(s e_{i}, 0\right)}(x), N(x)\right\rangle=-2\left\langle e_{i}, N(x)\right\rangle=-2 \psi_{i}(x)
$$

and so, from the second variation formula, we have

$$
\begin{aligned}
& \frac{d^{2}}{(d s)^{2}}{ }_{\mid s=0} f\left(s e_{i}, 0\right)=-4 \int_{\Sigma} \psi_{i} L \psi_{i} d \mu, \\
& {\frac{d^{2}}{(d s)^{2}}}_{\mid s=0} f(0, s)=-\int_{\Sigma} \psi_{5} L \psi_{5} d \mu .
\end{aligned}
$$

The fact that $f$ has a non-degenerate maximum at the origin follows at once from the following claim.

Claim: $-\int_{\Sigma} \psi L \psi d \mu<0$ for every $\psi \in E \backslash\{0\}$.

Because $f$ has a global maximum at the origin we have $D^{2} f(0,0) \leq 0$ and this means that

$$
-\int_{\Sigma} \psi L \psi d \mu \leq 0 \quad \text { for every } \psi \in E
$$

Suppose the claim is not true. We can find $\phi \in E \backslash\{0\}$ such that

$$
-\int_{\Sigma} \phi L \psi d \mu=-\int_{\Sigma} \psi L \phi d \mu=0 \quad \text { for every } \psi \in E \text {. }
$$




\section{Hence}

$$
\int_{\Sigma} \phi \psi_{i} d \mu=0 \quad \text { for every } 1 \leq i \leq 4, \quad \text { and } \quad \int_{\Sigma} L \phi d \mu=0 .
$$

This implies, since $\psi_{5}=1 \in E$, the existence of $c_{0}, \ldots, c_{4} \in \mathbb{R}$ such that

$$
1=c_{0} \phi+\psi, \quad \text { where } \quad \psi=\sum_{i=1}^{4} c_{i} \psi_{i} .
$$

Thus, because $L \psi=2 \psi$, we have

$$
\int_{\Sigma}\left(|A|^{2}+2\right) d \mu=\int_{\Sigma} L(1) d \mu=\int_{\Sigma}\left(c_{0} L \phi+L \psi\right) d \mu=2 \int_{\Sigma} \psi d \mu .
$$

On the other hand, we also have $1=c_{0}^{2} \phi^{2}+2 c_{0} \phi \psi+\psi^{2}$. If we integrate over $\Sigma$, we obtain from (19)

$$
\begin{array}{r}
\operatorname{area}(\Sigma)=\int_{\Sigma}\left(c_{0}^{2} \phi^{2}+2 c_{0} \phi \psi+\psi^{2}\right) d \mu \geq \int_{\Sigma} \psi^{2} d \mu=\int_{\Sigma} \psi\left(1-c_{0} \phi\right) d \mu \\
=\int_{\Sigma} \psi d \mu .
\end{array}
$$

Hence

$$
2 \operatorname{area}(\Sigma) \leq \int_{\Sigma}\left(|A|^{2}+2\right) d \mu=2 \int_{\Sigma} \psi d \mu \leq 2 \operatorname{area}(\Sigma) .
$$

This implies $A=0$ and so $\Sigma$ is a great sphere. This is a contradiction and so the claim holds.

Because we are assuming index $(\Sigma) \geq 6$, there exists $\varphi \in C^{\infty}(\Sigma)$ such that

- $-\int \varphi L \varphi d \mu<0$,

- $-\int \varphi L \psi_{i} d \mu=0$ for $1 \leq i \leq 5$.

Let $X$ be any vector field such that $X=\varphi N$ along $\Sigma$, and let $\left\{\Gamma_{s}\right\}_{s \geq 0}$ be the one parameter group of diffeomorphisms generated by $X$. We abuse notation and define $f: B_{\delta}^{4}(0) \times(-\delta, \delta) \times(-\delta, \delta) \rightarrow \mathbb{R}$ by

$$
f(v, t, s)=\mathbf{M}\left(\Gamma_{s} \circ P_{(v, t)}(\Sigma)\right) .
$$

We have $f(0,0,0)=\mathbf{M}(\Sigma)$, and $D f(0,0,0)=0$ since $\Sigma$ is minimal. It follows from the choice of $\varphi$, the second variation formula, and Lemma 5.6 that $D^{2} f(0,0,0)<0$. This means that there exists $0<\delta_{1} \leq \delta$ such that

$$
\operatorname{area}\left(\Gamma_{s} \circ P_{(v, t)}(\Sigma)\right)<f(0,0,0)=\operatorname{area}(\Sigma)
$$

for every $(v, t, s) \in\left(B_{\delta_{1}}^{4}(0) \times\left(-\delta_{1}, \delta_{1}\right) \times\left(-\delta_{1}, \delta_{1}\right)\right) \backslash\{(0,0,0)\}$.

Let $\beta: \mathbb{R}^{5} \rightarrow \mathbb{R}$ be a smooth function such that $0 \leq \beta(y) \leq \delta_{1} / 2$ for $y \in \mathbb{R}^{5}, \beta(y)=0$ if $|y| \geq \delta_{1} / 2$ and $\beta(y)=\delta_{1} / 2$ if $|y| \leq \delta_{1} / 4$. We then define

$$
C^{\prime}(v, t)=\Gamma_{\beta(v, t)} \circ P_{(v, t)}(\Sigma) \in \mathcal{Z}_{2}\left(S^{3}\right) \text { for }|(v, t)|<\delta_{1} .
$$


We have that $C^{\prime}(v, t)=C(v, t)$ if $\delta_{1} / 2<|(v, t)|<\delta_{1}$, where $C$ is the map given by Theorem 4.9, and this means we can extend $C^{\prime}$ to a continuous map in the flat topology

$$
C^{\prime}: \bar{B}^{4} \times[-\pi, \pi] \rightarrow \mathcal{Z}_{2}\left(S^{3}\right)
$$

by defining $C^{\prime}(v, t)=C(v, t)$ if $|(v, t)| \geq \delta_{1}$. Moreover, the map $C^{\prime}$ is homotopic with $C$ in the flat topology relative to the boundary of $\bar{B}^{4} \times[-\pi, \pi]$ and, from (20),

$$
\sup \left\{\mathbf{M}\left(C^{\prime}(v, t)\right):|(v, t)|<\delta_{1}\right\}<\mathbf{M}(\Sigma)=\mathbf{L}([\Phi]) .
$$

The procedure described in Section 4.12 gives us $\Phi^{\prime}: I^{5} \rightarrow \mathcal{Z}_{2}\left(S^{3}\right)$ that coincides with $\Phi$ outside a neighborhood of the center of $I^{5}$, belongs to $[\Phi]$ and, from Lemma 5.6 and the inequality above, that

$$
\mathbf{M}\left(\Phi^{\prime}(x)\right)<\mathbf{M}(\Sigma)=\mathbf{L}([\Phi]) \text { for all } x \in I^{5} .
$$

This completes the proof of Theorem 5.3 modulo the following technical comment.

5.7. Technical remark: Due to the fact that the mass is not continuous with respect to the flat topology, the inequality above does not imply that

$$
\sup _{x \in I^{5}} \mathbf{M}\left(\Phi^{\prime}(x)\right)<\mathbf{M}(\Sigma),
$$

which is needed to get a contradiction and conclude the proof of Theorem 5.3. In principle it could happen that for some $x_{i}$ tending $\partial I^{5}$ we have $\Phi^{\prime}\left(x_{i}\right)$ tending to a sphere in the flat topology but $\mathbf{M}\left(\Phi^{\prime}\left(x_{i}\right)\right)$ tends to $\mathbf{M}(\Sigma)$. The fact that this does not occur is proven in Section 10 of [31] and the reader can see there the details.

5.8. $2 \pi^{2}$-Theorem. Let $\Phi_{0}: \partial I^{5} \rightarrow \mathcal{Z}_{2}\left(S^{3} ; \mathbf{F}\right)$ be a continuous map in the $\mathbf{F}$-topology such that:

(1) $\Phi_{0}(x, 0)=\Phi_{0}(x, 1)=0$ for any $x \in I^{4}$;

(2) there is a map $Q: \partial I^{4} \rightarrow S^{3}$ so that

$$
\Phi_{0}(x, t)=\partial B_{t \pi}(Q(x)) \quad \text { for all }(x, t) \in \partial I^{4} \times I ;
$$

(3) the $\operatorname{map} Q: \partial I^{4} \rightarrow S^{3}$ has $\operatorname{deg}(Q) \neq 0$.

Every $\Phi \in \mathcal{E}\left(\Phi_{0}\right)$ has

$$
\sup \left\{\mathbf{M}(\Phi(x)): x \in I^{5}\right\} \geq 2 \pi^{2} .
$$

Proof. Given $\Phi \in \mathcal{E}\left(\Phi_{0}\right)$ we use Theorem 2.13 to obtain $\Pi_{\mathbf{M}}$ a $\left(I^{5}, \mathbf{M}\right)$ homotopy class of mappings into $\left(\mathcal{Z}_{2}\left(S^{3} ; \mathbf{M}\right), \Phi_{0}\right)$ such that

$$
\mathbf{L}([\Phi]) \leq \mathbf{L}\left(\Pi_{\mathbf{M}}\right) \leq \sup \left\{\mathbf{M}(\Phi(x)): x \in I^{5}\right\} .
$$


From Theorem 3.1 we know that $\mathbf{L}\left(\Pi_{\mathbf{M}}\right) \geq \mathbf{L}([\Phi])>4 \pi$ and so we can use Theorem 2.12 to conclude the existence of a minimal surface $S$ (with possible multiplicities) so that

$$
4 \pi<\mathbf{M}(S)=\mathbf{L}\left(\Pi_{\mathbf{M}}\right) \leq \sup \left\{\mathbf{M}(\Phi(x)): x \in I^{5}\right\} .
$$

Without loss of generality we can assume that $4 \pi<\mathbf{M}(S)<8 \pi$ and so $S$ is a minimal surface of positive genus. Theorem 5.3 implies that area $(S) \geq 2 \pi^{2}$ and this completes the proof.

\section{Application: The Willmore Conjecture}

We use the $2 \pi^{2}$-Theorem to show 1.

6.1. Theorem. Let $\Sigma \subset S^{3}$ be a closed embedded surface of genus $g \geq$

Then

$$
\mathcal{W}(\Sigma) \geq 2 \pi^{2}
$$

and the equality holds if and only if $\Sigma$ is the Clifford torus up to conformal transformations of $S^{3}$.

As we explained in Section 1, this implies the Willmore Conjecture.

Sketch of PRoof. Consider the family $\Phi: I^{5} \rightarrow \mathcal{Z}_{2}\left(S^{3}\right)$ associated to $\Sigma$ that is given by Theorem 4.1 and set $\Phi_{0}=\Phi_{\mid \partial I^{5}}$. Because the genus of $\Sigma$ is positive, we obtain from Theorem 4.1 that all conditions of the $2 \pi^{2}$-Theorem are satisfied and hence

$$
2 \pi^{2} \leq \sup \left\{\mathbf{M}(\Phi(x)): x \in I^{5}\right\} \leq \mathcal{W}(\Sigma),
$$

where the last inequality follows from Theorem 4.1 (3).

We leave the details of the rigidity statement for the reader to check in [31, Section 11] but the idea is the following. If $\Sigma$ has $\mathcal{W}(\Sigma)=2 \pi^{2}$, then the map $\Phi$ has

$$
2 \pi^{2} \leq \mathbf{L}([\Phi]) \leq \mathcal{W}(\Sigma)=2 \pi^{2}
$$

and so equalities must hold. Hence map $\Phi$ is optimal and we use this to show that $\Sigma$ is conformal to a minimal surface of area $2 \pi^{2}$, which we know it has to be the Clifford torus.

\section{Canonical family of links}

The material in this section and the next one corresponds to the joint work between the authors and Ian Agol [1].

Let $\gamma_{i}: S^{1} \rightarrow \mathbb{R}^{4}, i=1,2$, be a 2 -component link, i.e., a pair of rectifiable curves with $\gamma_{1}\left(S^{1}\right) \cap \gamma_{2}\left(S^{1}\right)=\emptyset$. After a reparametrization, we can assume $\gamma_{1}$ and $\gamma_{2}$ are Lipschitz and parametrized proportionally to arc length. The aim of this section is to construct a 5-parameter family $\Phi$ to which we can apply the $2 \pi^{2}$-Theorem. More precisely, we want to prove the following theorem, which was first shown in $[\mathbf{1}]$ : 
7.1. Theorem. Let $\left(\gamma_{1}, \gamma_{2}\right)$ be a 2-component link in $S^{3}$ with $\left|\operatorname{lk}\left(\gamma_{1}, \gamma_{2}\right)\right|=$ 1.

There is a continuous map in the flat topology $\Phi: I^{5} \rightarrow \mathcal{Z}_{2}\left(S^{3}\right)$ with no concentration of mass and satisfying the properties:

(1) $\Phi(x, 0)=\Phi(x, 1)=0$ for any $x \in I^{4}$;

(2) there is a map $Q: \partial I^{4} \rightarrow S^{3}$ so that

$$
\Phi(x, t)=\partial B_{t \pi}(Q(x)) \quad \text { for all }(x, t) \in \partial I^{4} \times I ;
$$

(3) $\sup \left\{\mathbf{M}(\Phi(x)): x \in I^{5}\right\} \leq E\left(\gamma_{1}, \gamma_{2}\right)$;

(4) the center map $Q: \partial I^{4} \rightarrow S^{3}$ satisfies $\operatorname{deg}(Q)=1$.

7.2. Gauss map. There is an interesting connection between links $\left(\gamma_{1}, \gamma_{2}\right)$ in $\mathbb{R}^{4}$ and surfaces in $S^{3}$ that we now explain.

The Gauss map of $\left(\gamma_{1}, \gamma_{2}\right)$ in $\mathbb{R}^{4}$, denoted by $g=G\left(\gamma_{1}, \gamma_{2}\right)$, is the Lipschitz map

$$
g: S^{1} \times S^{1} \rightarrow S^{3}, \quad g(s, t)=\frac{\gamma_{1}(s)-\gamma_{2}(t)}{\left|\gamma_{1}(s)-\gamma_{2}(t)\right|}
$$

One can then consider the current $g_{\#}\left(S^{1} \times S^{1}\right) \in \mathcal{Z}_{2}\left(S^{3}\right)$ defined by

$$
g_{\#}\left(S^{1} \times S^{1}\right)(\phi)=\int_{S^{1} \times S^{1}} G\left(\gamma_{1}, \gamma_{2}\right)^{*} \phi, \quad \phi \in \mathcal{D}^{2}\left(\mathbb{R}^{4}\right),
$$

where, recall, $\mathcal{D}^{2}\left(\mathbb{R}^{4}\right)$ denotes the space of smooth 2 -forms in $\mathbb{R}^{4}$ with compact support.

The next lemma shows that the mass of $g_{\#}\left(S^{1} \times S^{1}\right)$ is controlled by the Möbius energy of $\left(\gamma_{1}, \gamma_{2}\right)$.

7.3. Lemma. For almost every $(s, t) \in S^{1} \times S^{1}$,

$$
|\operatorname{Jac} g|(s, t) \leq \frac{\left|\gamma_{1}^{\prime}(s)\right|\left|\gamma_{2}^{\prime}(t)\right|}{\left|\gamma_{1}(s)-\gamma_{2}(t)\right|^{2}}
$$

and

$$
\mathbf{M}\left(g_{\#}\left(S^{1} \times S^{1}\right)\right) \leq \int_{S^{1} \times S^{1}}|\operatorname{Jac} g| d s d t \leq E\left(\gamma_{1}, \gamma_{2}\right) .
$$

Proof. We have

$\frac{\partial g}{\partial s}=\frac{1}{\left|\gamma_{1}-\gamma_{2}\right|}\left(\gamma_{1}^{\prime}-\left\langle g, \gamma_{1}^{\prime}\right\rangle g\right)$ and $\frac{\partial g}{\partial t}=-\frac{1}{\left|\gamma_{1}-\gamma_{2}\right|}\left(\gamma_{2}^{\prime}-\left\langle g, \gamma_{2}^{\prime}\right\rangle g\right)$.

Thus

$$
\begin{aligned}
\left|\frac{\partial g}{\partial s}\right|^{2}\left|\frac{\partial g}{\partial t}\right|^{2}-\left\langle\frac{\partial g}{\partial s}, \frac{\partial g}{\partial t}\right\rangle^{2} & \leq\left|\frac{\partial g}{\partial s}\right|^{2}\left|\frac{\partial g}{\partial t}\right|^{2} \\
& =\frac{\left|\gamma_{1}^{\prime}\right|^{2}-\left\langle g, \gamma_{1}^{\prime}\right\rangle^{2}}{\left|\gamma_{1}-\gamma_{2}\right|^{2}} \frac{\left|\gamma_{2}^{\prime}\right|^{2}-\left\langle g, \gamma_{2}^{\prime}\right\rangle^{2}}{\left|\gamma_{1}-\gamma_{2}\right|^{2}} \leq \frac{\left|\gamma_{1}^{\prime}\right|^{2}\left|\gamma_{2}^{\prime}\right|^{2}}{\left|\gamma_{1}-\gamma_{2}\right|^{4}}
\end{aligned}
$$


and this proves the first inequality in the lemma. The second inequality follows because

$\mathbf{M}\left(g_{\#}\left(S^{1} \times S^{1}\right)\right) \leq \int_{S^{1} \times S^{1}}|\operatorname{Jac} g| d s d t \leq \int_{S^{1} \times S^{1}} \frac{\left|\gamma_{1}^{\prime}\right|^{2}\left|\gamma_{2}^{\prime}\right|^{2}}{\left|\gamma_{1}-\gamma_{2}\right|^{4}} d s d t=E\left(\gamma_{1}, \gamma_{2}\right)$

7.4. Canonical family. Given $v \in \mathbb{R}^{4}$, we define the conformal map

$$
F_{v}: \mathbb{R}^{4} \backslash\{v\} \rightarrow \mathbb{R}^{4}, \quad F_{v}(x)=\frac{x-v}{|x-v|^{2}}
$$

For every $r>0$ and $x \in \mathbb{R}^{4}$ we denote $S_{r}^{3}(x)=\partial B_{r}^{4}(x)$. If $v \in B^{4}$ we have that

$$
F_{v}\left(S_{1}^{3}(0)\right)=S_{\frac{1}{1-|v|^{2}}}^{3}(c(v)) \quad \text { where } \quad c(v)=\frac{v}{1-|v|^{2}} .
$$

Given $w \in \mathbb{R}^{4}$ and $\lambda \in \mathbb{R}$, we set $D_{w, \lambda}(x)=\lambda(x-w)+w$, where $x \in \mathbb{R}^{4}$.

Finally, given $v \in \bar{B}^{4}$ and $z \in(0,1)$, we also define

$$
a(v, z)=1+\frac{\left(1-|v|^{2}\right)(2 z-1)}{\left(1-|v|^{2}+z\right)(1-z)} .
$$

For each $v \in B^{4}$ fixed, $z \rightarrow a(v, z)$ is a nondecreasing parametrization of $(0,+\infty)$ where $a(v, 1 / 2)=1$.

Suppose now that $\gamma_{1}\left(S^{1}\right) \cup \gamma_{2}\left(S^{1}\right) \subset S^{3}$. The canonical five-dimensional family of surfaces associated to $\left(\gamma_{1}, \gamma_{2}\right)$ is given by

$$
C: B^{4} \times(0,1) \rightarrow \mathcal{Z}_{2}\left(S^{3}\right), \quad C(v, z)=g_{(v, z) \#}\left(S^{1} \times S^{1}\right) \in \mathcal{Z}_{2}\left(S^{3}\right),
$$

where $g_{(v, z)}: S^{1} \times S^{1} \rightarrow S^{3}$ is defined by

$$
g_{(v, z)}(s, t)=\frac{\left(F_{v} \circ \gamma_{1}\right)(s)-\left(D_{c(v), a(v, z)} \circ F_{v} \circ \gamma_{2}\right)(t)}{\left|\left(F_{v} \circ \gamma_{1}\right)(s)-\left(D_{c(v), a(v, z)} \circ F_{v} \circ \gamma_{2}\right)(t)\right|} .
$$

Note that $g_{(v, z)}=G\left(F_{v} \circ \gamma_{1}, D_{c(v), a(v, z)} \circ F_{v} \circ \gamma_{2}\right)$. Furtermore, $g_{(v, 1 / 2)}=$ $G\left(F_{v} \circ \gamma_{1}, F_{v} \circ \gamma_{2}\right)$, since $a(v, 1 / 2)=1$.

The following proposition is the analogue of Theorem 4.4.

7.5. Proposition. For every $(v, z) \in B^{4} \times(0,1)$,

$$
\mathbf{M}(C(v, z)) \leq E\left(\gamma_{1}, \gamma_{2}\right) \text {. }
$$

Proof. An explicit computation [1, Lemma 2.4] shows

$$
\left|F_{v} \circ \gamma_{1}-D_{c(v), a(v, z)} \circ F_{v} \circ \gamma_{2}\right|^{2}=a(v, z)\left|F_{v} \circ \gamma_{1}-F_{v} \circ \gamma_{2}\right|^{2}+b(v, z)^{2} .
$$

Moreover

$$
\left|\left(D_{c(v), a(v, z)} \circ F_{v} \circ \gamma_{2}\right)^{\prime}(t)\right|=a(v, z)\left|\left(F_{v} \circ \gamma_{2}\right)^{\prime}(t)\right| .
$$

Thus, combining with Lemma 7.3, we obtain for almost all $(s, t) \in S^{1} \times S^{1}$ 


$$
\begin{aligned}
\left|\operatorname{Jac} g_{(v, z)}\right|(s, t) & \leq \frac{\left|\left(F_{v} \circ \gamma_{1}\right)^{\prime}(s)\right|\left|\left(D_{c(v), a(v, z)} \circ F_{v} \circ \gamma_{2}\right)^{\prime}(t)\right|}{\left|F_{v} \circ \gamma_{1}(s)-D_{c(v), a(v, z)} \circ F_{v} \circ \gamma_{2}(t)\right|^{2}} \\
& \leq \frac{a(v, z)\left|\left(F_{v} \circ \gamma_{1}\right)^{\prime}(s)\right|\left|\left(F_{v} \circ \gamma_{2}\right)^{\prime}(t)\right|}{a(v, z)\left|F_{v} \circ \gamma_{1}(s)-F_{v} \circ \gamma_{2}(t)\right|^{2}+b(v, z)^{2}} \\
& \leq \frac{\left|\left(F_{v} \circ \gamma_{1}\right)^{\prime}(s)\right|\left|\left(F_{v} \circ \gamma_{2}\right)^{\prime}(t)\right|}{\left|F_{v} \circ \gamma_{1}(s)-F_{v} \circ \gamma_{2}(t)\right|^{2}} .
\end{aligned}
$$

Hence, using the conformal invariance of the Möbius energy,

$$
\begin{array}{r}
\mathbf{M}(C(v, z)) \leq \int_{S^{1} \times S^{1}} \frac{\left|\left(F_{v} \circ \gamma_{1}\right)^{\prime}(s)\right|\left|\left(F_{v} \circ \gamma_{2}\right)^{\prime}(t)\right|}{\left|F_{v} \circ \gamma_{1}(s)-F_{v} \circ \gamma_{2}(t)\right|^{2}} d s d t \\
\quad=E\left(F_{v} \circ \gamma_{1}, F_{v} \circ \gamma_{2}\right)=E\left(\gamma_{1}, \gamma_{2}\right) .
\end{array}
$$

7.6. Continuity of the family. We analyze the continuity of $C$ defined in the previous section. In order to do that, we first extend the definition of $g_{(v, z)}$ to hold for all $(v, z) \in \bar{B}^{4} \times[0,1]$.

We start with the following remark. Choose $\left\{v_{i}\right\}_{i \in \mathbb{N}}$ a sequence in $B^{4}$ tending to $v \in S^{3}$. Then for every $x \in S^{3} \backslash\{v\}$ and $0<z<1$ we have

$$
\begin{aligned}
\lim _{i \rightarrow \infty} D_{c\left(v_{i}\right), a\left(v_{i}, z\right)} \circ F_{v_{i}}(x)=\lim _{i \rightarrow \infty} a\left(v_{i}, z\right) F_{v_{i}}(x)- & v_{i} \frac{a\left(v_{i}, z\right)-1}{1-\left|v_{i}\right|^{2}} \\
& =F_{v}(x)-\frac{2 z-1}{z(1-z)} v .
\end{aligned}
$$

In light of this we define, for every $0<z<1$, the hyperplane

$$
P_{(v, z)}=\left\{x \in \mathbb{R}^{4}:\langle x, v\rangle=-1 / 2-b(z)\right\}, \quad \text { where } \quad b(z)=\frac{2 z-1}{z(1-z)}
$$

and the smooth map

$$
L_{(v, z)}: S^{3} \backslash\{v\} \rightarrow P_{(v, z)}, \quad L_{(v, z)}(x)=F_{v}(x)-b(z) v .
$$

Note that indeed

$$
\left\langle L_{(v, z)}(x), v\right\rangle=\left\langle\frac{x-v}{|x-v|^{2}}, v\right\rangle-b(z)=\frac{\langle x, v\rangle-1}{2-2\langle x, v\rangle}-b(z)=-1 / 2-b(z) .
$$

Thus, with

$$
S_{v}=\left(\gamma_{1}^{-1}(v) \times S^{1}\right) \cup\left(S^{1} \times \gamma_{2}^{-1}(v)\right)
$$

(which is empty if $v \notin \gamma_{1} \cup \gamma_{2}$ ) we set for $v \in S^{3}$ and $0<z<1$

$$
g_{(v, z)}:\left(S^{1} \times S^{1}\right) \backslash S_{v} \rightarrow S^{3}, \quad g_{(v, z)}=G\left(F_{v} \circ \gamma_{1}, L_{(v, z)} \circ \gamma_{2}\right) .
$$


7.7. Proposition. The canonical family can be extended to a continuous map in the flat metric

$$
C: \bar{B}^{4} \times[0,1] \rightarrow \mathcal{Z}_{2}\left(S^{3}\right)
$$

with the following properties:

(i) $C(v, 0)=C(v, 1)=0$ for all $v \in \bar{B}^{4}$.

(ii) If $v \in S^{3} \backslash\left(\gamma_{1}\left(S^{1}\right) \cup \gamma_{2}\left(S^{1}\right)\right)$ and $0<z<1$ then

$$
C(v, z)=g_{(v, z)_{\#}}\left(S^{1} \times S^{1}\right) .
$$

Sketch of Proof. This result was proven in [1, Proposition 3.1] and so we just present the main ideas.

First we showed that the set $S_{v} \subset S^{1} \times S^{1}$ has measure zero for all $v \in S^{3}$.

Moreover, if $\left(v_{i}, z_{i}\right) \in B^{4} \times(0,1)$ tends to $(v, z) \in \bar{B}^{4} \times(0,1)$, it was also shown that $g_{\left(v_{i}, z_{i}\right)}$ and $D g_{\left(v_{i}, z_{i}\right)}$ converges pointwise, respectively, to the Lipschitz map $g_{(v, z)}$ and $D g_{(v, z)}$ almost everywhere. Furthermore, in Lemma 2.6 of [1] it was shown the existence of a constant $C>0$ such that for all $(v, z) \in B^{4} \times(0,1)$ we have $\left|\operatorname{Jac} g_{(v, z)}\right|(s, t) \leq C$ almost everywhere.

Hence, from the Lebesgue's Dominated Convergence Theorem, we have that for all $\phi \in \mathcal{D}^{2}\left(\mathbb{R}^{4}\right)$

$$
\lim _{i \rightarrow \infty} C\left(v_{i}, z_{i}\right)(\phi)=\lim _{i \rightarrow \infty} \int_{S^{1} \times S^{1}} g_{\left(v_{i}, z_{i}\right)}^{*}(\phi)=\int_{\left(S^{1} \times S^{1}\right) \backslash S_{v}} g_{(v, z)}^{*}(\phi) .
$$

In Proposition 3.1 of $[\mathbf{1}]$ we used this identity to show that the map $C$ can be continuously extended to $\bar{B}^{4} \times(0,1)$.

Proposition 7.7 (i) follows from the fact that, with $v \in B^{4}$ fixed and $z$ tending to 0 or $1, g_{(v, z)}$ tends, respectively, to the map

$$
(s, t) \mapsto \frac{F_{v} \circ \gamma_{1}(s)-c(v)}{\left|F_{v} \circ \gamma_{1}(s)-c(v)\right|} \quad \text { or } \quad(s, t) \mapsto-\frac{F_{v} \circ \gamma_{2}(t)-c(v)}{\left|F_{v} \circ \gamma_{2}(t)-c(v)\right|},
$$

which has vanishing Jacobian.

7.8. Boundary behavior. Like in Section 4.5 when one was studying the canonical family of surfaces, it is important that the map $C$ when restricted to $\partial\left(B^{4} \times(0,1)\right)$ detects topological information.

7.9. Proposition. There exists a constant $c>0$ such that for every $p \in S^{3}$ we have

(i) $C(p, 1 / 2)=-\operatorname{lk}\left(\gamma_{1}, \gamma_{2}\right) \cdot \partial B_{\pi / 2}(p)$,

(ii) $\operatorname{supp}(C(p, z)) \subset \bar{B}_{\pi / 2}(p) \backslash B_{r(z)}(p)$ if $z \in[1 / 2,1]$,

(iii) $\operatorname{supp}(C(p, z)) \subset \bar{B}_{\pi / 2}(-p) \backslash B_{\pi-r(z)}(-p)$ if $z \in[0,1 / 2]$,

where

$$
r(z)=\cos ^{-1}\left(\frac{b(z)}{\sqrt{|b(z)|^{2}+c^{2}}}\right) \in[0, \pi] \quad \text { and } \quad b(z)=\frac{2 z-1}{z(1-z)} .
$$


Notice that $r(0)=\pi, r(1 / 2)=\pi / 2$, and $r(1)=0$.

Proof. Given $p \in S^{3} \backslash\left(\gamma_{1}\left(S^{1}\right) \cup \gamma_{2}\left(S^{1}\right)\right),\left(F_{p} \circ \gamma_{1}, F_{p} \circ \gamma_{2}\right)$ is a link in the affine hyperplane

$$
P_{(p, 1 / 2)}=\left\{x \in \mathbb{R}^{4}:\langle x, p\rangle=-1 / 2\right\},
$$

where $F_{p}$ sends the exterior unit normal of $S^{3}$ into $p$. This implies that

$$
\operatorname{supp} C(p, 1 / 2) \subset G\left(F_{p} \circ \gamma_{1}, F_{p} \circ \gamma_{2}\right)\left(S^{1} \times S^{1}\right) \subset \partial B_{\pi / 2}(-p),
$$

and so by the Constancy Theorem [41, Theorem 26.27] we have that

$$
C(p, 1 / 2)=k \cdot \partial B_{\pi / 2}(-p)
$$

for some integer $k$.

Let $P$ be the oriented hyperplane with normal vector $p \in S^{3}$ and let $\omega_{P}$ be its volume form. Let $\omega, \omega_{S^{3}}, \omega_{\mathbb{R}^{4}}$ denote, respectively, the volume form of $\partial B_{\pi / 2}(-p) \subset S^{3}$ (the exterior unit normal is $\left.p\right), S^{3}$, and $\mathbb{R}^{4}$. We also have

$$
\partial B_{\pi / 2}(-p)=\left\{x \in \mathbb{R}^{4}:\langle x, p\rangle=0\right\} \cap S^{3} \subset P .
$$

With $g=G\left(\tilde{\gamma}_{1}, \tilde{\gamma}_{2}\right)$ and $\tilde{\gamma}_{i}=F_{p} \circ \gamma_{i}, i=1,2$, we have

$$
\begin{aligned}
g^{*} \omega\left(\frac{\partial}{\partial s}, \frac{\partial}{\partial t}\right) & =\omega\left(\frac{\partial g}{\partial s}, \frac{\partial g}{\partial t}\right)=\omega_{S^{3}}\left(\frac{\partial g}{\partial s}, \frac{\partial g}{\partial t}, p\right) \\
& =\omega_{\mathbb{R}^{4}}\left(\frac{\partial g}{\partial s}, \frac{\partial g}{\partial t}, p, g\right)=-\omega_{\mathbb{R}^{4}}\left(\frac{\partial g}{\partial s}, \frac{\partial g}{\partial t}, g, p\right) \\
& =-\omega_{P}\left(\frac{\partial g}{\partial s}, \frac{\partial g}{\partial t}, g\right)=-\operatorname{det}\left(\frac{\partial g}{\partial s}, \frac{\partial g}{\partial t}, g\right) \\
& =\frac{\operatorname{det}\left(\tilde{\gamma}_{1}^{\prime}, \tilde{\gamma}_{2}^{\prime}, \tilde{\gamma}_{1}-\tilde{\gamma}_{2}\right)}{\left|\tilde{\gamma}_{1}-\tilde{\gamma}_{2}\right|^{3}},
\end{aligned}
$$

and so, using Gauss formula (3) to compute linking numbers, we have

$$
\begin{aligned}
k=\frac{1}{4 \pi} C & (p, 1 / 2)(\omega)=\frac{1}{4 \pi} \int_{S^{1} \times S^{1}} g^{*} \omega \\
= & \frac{1}{4 \pi} \int_{S^{1} \times S^{1}} \frac{\operatorname{det}\left(\tilde{\gamma}_{1}^{\prime}, \tilde{\gamma}_{2}^{\prime}, \tilde{\gamma}_{1}-\tilde{\gamma}_{2}\right)}{\left|\tilde{\gamma}_{1}-\tilde{\gamma}_{2}\right|^{3}} d s d t=\operatorname{lk}\left(\tilde{\gamma}_{1}, \tilde{\gamma}_{2}\right)=\operatorname{lk}\left(\gamma_{1}, \gamma_{2}\right) .
\end{aligned}
$$

By continuity of $C$, we also have $C(p, 1 / 2)=-\operatorname{lk}\left(\gamma_{1}, \gamma_{2}\right) \cdot \partial B_{\pi / 2}(p)$ for $p \in \gamma_{1}\left(S^{1}\right) \cup \gamma_{2}\left(S^{1}\right)$. This proves item (i).

We now prove Proposition 7.9 (ii) and (iii). If $z=0$ or $z=1$, we have $C(p, z)=0$ and so (ii) and (iii) hold. Suppose $z \in(0,1)$ and $p \in$ $S^{3} \backslash\left(\gamma_{1}\left(S^{1}\right) \cup \gamma_{2}\left(S^{1}\right)\right)$. Then from Proposition 7.7 we have

$$
\operatorname{supp}(C(p, z)) \subset g_{(p, z)}\left(S^{1} \times S^{1}\right) .
$$

We first argue that $C(p, z)$ lies in $\bar{B}_{\pi / 2}(p)$ for $z \in[1 / 2,1]$ and in $\bar{B}_{\pi / 2}(-p)$ for $z \in[0,1 / 2]$. From $(21)$ and the fact that $\left(F_{p} \circ \gamma_{1}, F_{p} \circ \gamma_{2}\right)$ lies in $P_{(p, 1 / 2)}$ 
we have

$$
\begin{aligned}
\left\langle g_{(p, z)}, p\right\rangle & =\left\langle\frac{F_{p} \circ \gamma_{1}-L_{(p, z)} \circ \gamma_{2}}{\left|F_{p} \circ \gamma_{1}-L_{(p, z)} \circ \gamma_{2}\right|}, p\right\rangle \\
& =\left\langle\frac{F_{p} \circ \gamma_{1}+\frac{1}{2} p-\left(L_{(p, z)} \circ \gamma_{2}+\frac{1}{2} p\right)}{\left|F_{p} \circ \gamma_{1}-L_{(p, z)} \circ \gamma_{2}\right|}, p\right\rangle \\
& =\frac{b(z)}{\left|F_{p} \circ \gamma_{1}-L_{(p, z)} \circ \gamma_{2}\right|} .
\end{aligned}
$$

Hence $\left\langle g_{(p, z)}, p\right\rangle \geq 0$ if $z \in[1 / 2,1]$, and $\left\langle g_{(p, z)}, p\right\rangle \leq 0$ if $z \in[0,1 / 2]$.

Using the fact that for some constant $c>0$ we have (see $[\mathbf{1}$, Proposition 4.1])

$$
\left|F_{p} \circ \gamma_{1}(s)-F_{p} \circ \gamma_{2}(t)\right|^{2} \geq c^{2}
$$

for all $p \in S^{3}$ and $(s, t) \in S^{1} \times S^{1}$, it follows that

$$
\left|\left\langle g_{(p, z)}(s, t), p\right\rangle\right| \leq \frac{|b(z)|}{\sqrt{c^{2}+b(z)^{2}}} .
$$

This proves items (ii) and (iii) of the proposition for $z \in[0,1]$ and $p \in$ $S^{3} \backslash\left(\gamma_{1}\left(S^{1}\right) \cup \gamma_{2}\left(S^{1}\right)\right)$.

Since $S^{3} \backslash\left(\gamma_{1}\left(S^{1}\right) \cup \gamma_{2}\left(S^{1}\right)\right)$ is everywhere dense in $S^{3}$, and $C: S^{3} \times$ $[0,1] \rightarrow \mathcal{Z}_{2}\left(S^{3}\right)$ is continuous in the flat topology, the proposition also holds for $p \in \gamma_{1}\left(S^{1}\right) \cup \gamma_{2}\left(S^{1}\right)$.

7.10. Sketch of proof of Theorem 7.1. Without loss of generality we assume that $\operatorname{lk}\left(\gamma_{1}, \gamma_{2}\right)=-1$.

The map $C$ does not have the property that $C(v, z)$ is a round sphere when $(v, z) \in S^{3} \times[0,1]$. Nonetheless, the information given by the previous proposition is enough to find an extension of $C$ having that property and this is what we explain now.

Let $p \in S^{3}, \lambda \in[0, \pi / 2]$. For $t \in[0,1]$, we define a retraction map

$$
R_{(p, \lambda, t)}: \bar{B}_{\pi / 2}(p) \backslash B_{\lambda}(p) \rightarrow \bar{B}_{\pi / 2}(p) \backslash B_{\lambda}(p)
$$

by

$$
R_{(p, \lambda, t)}(x)=\exp _{p}\left(\left((1-t)+t \frac{\lambda}{d(p, x)}\right) \exp _{p}^{-1}(x)\right),
$$

where the geometric quantities are computed with respect to the canonical metric on $S^{3}$. Notice that $R_{(p, \pi / 2, t)}: \partial B_{\pi / 2}(p) \rightarrow \partial B_{\pi / 2}(p)$ is the identity map for every $p \in S^{3}$ and $t \in[0,1]$. In Proposition 4.2 of $[\mathbf{1}]$ we show that $R_{(p, \lambda, t)}$ are length-decreasing maps that satisfy

- $R_{(p, \lambda, 0)}(x)=x$ for all $x \in \bar{B}_{\pi / 2}(p) \backslash B_{\lambda}(p)$,

- $R_{(p, \lambda, 1)}\left(\bar{B}_{\pi / 2}(p) \backslash B_{\lambda}(p)\right) \subset \partial B_{\lambda}(p)$. 
We now define $\tilde{C}: \bar{B}_{2}^{4}(0) \times[0,1] \rightarrow \mathcal{Z}_{2}\left(S^{3}\right)$ by

$$
\tilde{C}(v, z)=\left\{\begin{array}{lr}
C(v, z) & \text { if } v \in \bar{B}_{1}^{4}(0) \\
R\left(-\frac{v}{|v|}, \pi-r(z),|v|-1\right)_{\#} C\left(\frac{v}{|v|}, z\right) & \text { and } t \in[0,1], \\
R\left(\frac{v}{|v|}, r(z),|v|-1\right)_{\#} C\left(\frac{v}{|v|}, z\right) & \text { if } v \in \bar{B}_{2}^{4}(0) \backslash \bar{B}_{1}^{4}(0) \\
& \text { and } z \in[0,1 / 2), \\
2(0) \backslash \bar{B}_{1}^{4}(0)
\end{array}\right.
$$

where the function $r$ is the one given in Proposition 7.9.

Choose an orientation-preserving homeomorphism $f: I^{4} \rightarrow \bar{B}_{2}^{4}(0)$ and set

$$
\Phi: I^{5} \rightarrow \mathcal{Z}_{2}\left(S^{3}\right), \quad \Phi(x, t)=\tilde{C}\left(f(x), r^{-1}(t \pi)\right) .
$$

One can check that $\tilde{C}$ is continuous (see [1, Proposition 4.3] for details) and so $\Phi$ is continuous as well. Theorem 7.1 (1) follows from Proposition 7.7 (i).

If $v \in \partial \bar{B}_{2}^{4}(0)$, then $\operatorname{supp}(\tilde{C}(v, z)) \subset \partial B_{r(z)}\left(\frac{v}{|v|}\right)$. By the Constancy Theorem there exists $k(v, z) \in \mathbb{Z}$ such that

$$
\tilde{C}(v, z)=k(v, z) \cdot \partial B_{r(z)}\left(\frac{v}{|v|}\right) .
$$

Because of the continuity of $\tilde{C}$ in the flat topology, there must exist $k \in \mathbb{Z}$ such that $k(v, z)=k$ for every $v \in \partial B_{2}^{4}(0)$ and $z \in[0,1]$.

Next, we argue that $k=-\mathrm{lk}\left(\gamma_{1}, \gamma_{2}\right)=1$ and this suffices to show Theorem $7.1(2)$. Indeed, given $p \in S^{3}$ we have $C(p, 1 / 2)=-\operatorname{lk}\left(\gamma_{1}, \gamma_{2}\right) \cdot \partial B_{\pi / 2}\left(\frac{v}{|v|}\right)$ by Proposition 7.9 (i) and so

$$
\begin{aligned}
\tilde{C}(2 p, 1 / 2) & =R_{(p, r(1 / 2), 1)_{\#}}(C(p, 1 / 2)) \\
& =R_{(p, \pi / 2,1)_{\#}}\left(\partial B_{\pi / 2}(p)\right) \\
& =i d_{\#}\left(\partial B_{\pi / 2}(p)\right)=\partial B_{\pi / 2}(p) .
\end{aligned}
$$

Theorem 7.1 (3) follows from Proposition 7.5 and the fact that, because $R_{(p, \lambda, t)}$ are length-decreasing, then

$$
\mathbf{M}(\tilde{C}(v, z)) \leq \mathbf{M}\left(\tilde{C}\left(\frac{v}{|v|}, z\right)\right) \quad \text { for all } v \in \bar{B}_{2}^{4}(0) \backslash \bar{B}_{1}^{4}(0), \quad 0 \leq z \leq 1 .
$$

Finally, Theorem 7.1 (4) follows because $Q(x)=f(x)$ when $x \in \partial I^{4}$ and $f_{\mid \partial I^{4}}$ is a homemorphism into $S^{3}$.

\section{Application: Freedman-He-Wang Conjecture}

We have now all the available tools to prove the following result that, as it was explained in Section 1.7, confirms a conjecture of Freedman, He, and Wang. 
8.1. Theorem. Assume $\left(\gamma_{1}, \gamma_{2}\right) \subset S^{3}$ is a 2 -component link with $\left|\operatorname{lk}\left(\gamma_{1}, \gamma_{2}\right)\right|=$ 1. Then $E\left(\gamma_{1}, \gamma_{2}\right) \geq 2 \pi^{2}$.

Moreover, if $E\left(\gamma_{1}, \gamma_{2}\right)=2 \pi^{2}$ then $\left(\gamma_{1}, \gamma_{2}\right)$ is conformal to the standard Hopf link up to orientation and reparameterization.

SkEtCH OF PROOF. We prove only the inequality. The rigidity statement is proven in $[\mathbf{1}]$.

Consider the family $\Phi: I^{5} \rightarrow \mathcal{Z}_{2}\left(S^{3}\right)$ associated to $\left(\gamma_{1}, \gamma_{2}\right)$ that is given by Theorem 7.1 and set $\Phi_{0}=\Phi_{\mid \partial I^{5}}$. We obtain from Theorem 7.1 that all conditions of the $2 \pi^{2}$-Theorem are satisfied and hence

$$
2 \pi^{2} \leq \sup \left\{\mathbf{M}(\Phi(x)): x \in I^{5}\right\} \leq E\left(\gamma_{1}, \gamma_{2}\right) .
$$

where the last inequality follows from Theorem 7.1 (3).

\section{Gromov-Guth families}

In what follows $(M, g)$ is a compact Riemannian $(n+1)$-manifold and $X$ denotes a cubical subcomplex of $I^{m}=[0,1]^{m}$, for some $m$.

Almgren $[\mathbf{2}]$ showed that $\mathcal{Z}_{n}\left(M ; \mathbb{Z}_{2}\right)$ has all homotopy groups vanishing except the first one, which is $\mathbb{Z}_{2}$, and so it is weakly homotopic to $\mathbb{R P}^{\infty}$. Thus its cohomology ring has a single generator $\bar{\lambda} \in H^{1}\left(\mathcal{Z}_{n}\left(M ; \mathbb{Z}_{2}\right), \mathbb{Z}_{2}\right)$.

Denoting by $\bar{\lambda}^{p}$ the cup product of $\bar{\lambda}$ with itself $p$-times, Guth [20] and Gromov $[17,18,19]$ studied continuous maps $\Phi$ from a cellular complex $X$ into $\mathcal{Z}_{n}\left(M ; \mathbb{Z}_{2}\right)$ that detect $\bar{\lambda}^{p}$ i.e., $\Phi^{*}\left(\bar{\lambda}^{p}\right) \neq 0$. We now describe some of their results.

Recall the definition of sweepout in Section 2.18.

9.1. p-sweepout. A continuous map in the flat topology $\Phi: X \rightarrow$ $\mathcal{Z}_{n}\left(M ; \mathbb{Z}_{2}\right)$ is a $p$-sweepout if

$$
\Phi^{*}\left(\bar{\lambda}^{p}\right) \neq 0 \in H^{p}\left(X ; \mathbb{Z}_{2}\right) .
$$

This is equivalent to say that there exists $\lambda \in H^{1}\left(X ; \mathbb{Z}_{2}\right)$ such that:

(i) for any curve $\gamma: S^{1} \rightarrow X$, we have $\lambda(\gamma) \neq 0$ if and only if $\Phi \circ \gamma$ : $S^{1} \rightarrow \mathcal{Z}_{n}\left(M ; \mathbb{Z}_{2}\right)$ is a sweepout;

(ii) the cup product $\lambda^{p}=\lambda \smile \ldots \smile \lambda$ is nonzero in $H^{p}\left(X ; \mathbb{Z}_{2}\right)$.

\subsection{Remark:}

(1) A continuous map in the flat topology homotopic to a $p$-sweepout is also a $p$-sweepout.

(2) If $\gamma, \gamma^{\prime}$ are homotopic to each other in $X$, then $\Phi \circ \gamma$ is a sweepout if and only if $\Phi \circ \gamma^{\prime}$ is a sweepout. This will be useful to check condition (i) above on specific examples.

Informally, one can think of a $p$-sweepout as a map $\Phi$ with the following property: For every set $\left\{x_{1}, \ldots, x_{p}\right\} \subset M$, there is $\theta \in X$ so that $\left\{x_{1}, \ldots, x_{p}\right\} \subset \Phi(\theta)$.

We say $X$ is $p$-admissible if there is a $p$-sweepout $\Phi: X \rightarrow \mathcal{Z}_{n}\left(M ; \mathbb{Z}_{2}\right)$ that has no concentration of mass. The set of all $p$-sweepouts $\Phi$ that have 
no concentration of mass is denoted by $\mathcal{P}_{p}$. Note that two maps in $\mathcal{P}_{p}$ can have different domains.

Similarly to Guth [20, Appendix 3], we define the $p$-width of $M$ as

$$
\omega_{p}(M)=\inf _{\Phi \in \mathcal{P}_{p}} \sup \{\mathbf{M}(\Phi(x)): x \in \operatorname{dmn}(\Phi)\}
$$

where $\operatorname{dmn}(\Phi)$ is the domain of $\Phi$.

Notice that if a map $\Phi: X \rightarrow \mathcal{Z}_{n}\left(M ; \mathbb{Z}_{2}\right)$ is a $p$-sweepout, then it is also a $q$-sweepout for every $q \leq p$. Hence $\omega_{p}(M) \leq \omega_{p+1}(M)$ for every $p \in \mathbb{N}$.

The next result was proven by Gromov (see [17, Section 4.2.B] or [18, Section 8]) and later Guth [20] used a bend-and-cancel argument to give a somewhat different proof.

9.3. Theorem. For each $p \in \mathbb{N}$, the set $\mathcal{P}_{p}$ is non-empty.

Moreover, there exists a constant $C=C(M, g)>0$ so that, for every $p \in \mathbb{N}$,

$$
C^{-1} p^{\frac{1}{n+1}} \leq \omega_{p}(M) \leq C p^{\frac{1}{n+1}}
$$

9.4. Remark: We only present the proof of the upper bound estimate because that suffices for the application discussed in the next section. The reader can find the proof for the lower bound estimate in [17, Section 4.2.B], $[\mathbf{2 0}$, Section 3], or $[\mathbf{3 0}$, Section 8]

Proof of UPPer Bound estimate. We follow Guth's presentation [20].

First we show that $\mathcal{P}_{p} \neq \emptyset$. Let $f \in C^{\infty}(M)$ be a Morse function. We then define a function

$$
\hat{\Psi}_{p}:\left\{a \in \mathbb{R}^{p+1}:|a|=1\right\} \rightarrow \mathcal{Z}_{n}\left(M ; \mathbb{Z}_{2}\right)
$$

by

$$
\hat{\Psi}_{p}\left(a_{0}, \ldots, a_{p}\right)=\partial\left\{x \in M: a_{0}+a_{1} f(x)+\ldots+a_{p} f^{p}(x)<0\right\} .
$$

The set $\left\{x \in M: a_{0}+a_{1} f(x)+\ldots+a_{p} f^{p}(x)<0\right\}$ has finite perimeter and so $\hat{\Psi}_{p}\left(a_{0}, \ldots, a_{p}\right)$ is well defined. The fact that we are using $\mathbb{Z}_{2}$ coefficients implies that $\hat{\Psi}_{p}(a)=\hat{\Psi}_{p}(-a)$, and therefore $\hat{\Psi}_{p}$ induces a map

$$
\Psi_{p}: \mathbb{R} \mathbb{P}^{p} \rightarrow \mathcal{Z}_{n}\left(M ; \mathbb{Z}_{2}\right) .
$$

This map is continuous in the flat topology and has no concentration of mass (see [30, Section 5] for details). The curve

$$
\gamma: S^{1} \rightarrow \mathbb{R P}^{p}, \quad e^{i \theta} \mapsto[(\cos (\theta / 2), \sin (\theta / 2), 0, \ldots, 0)],
$$

is a generator of $\pi_{1}\left(\mathbb{R} P^{p}\right)$. Then

$$
\Psi_{p} \circ \gamma: S^{1} \rightarrow \mathcal{Z}_{n}\left(M ; \mathbb{Z}_{2}\right), \quad e^{i \theta} \mapsto \partial\{x \in M: f(x)<-\cot (\theta / 2)\},
$$

is a sweepout of $M$. The generator $\lambda \in H^{1}\left(\mathbb{R P P}^{p} ; \mathbb{Z}_{2}\right)$ satisfies $\lambda(\gamma)=1$, $\lambda^{p} \neq 0$, and so $\Psi_{p}$ is a $p$-sweepout. In particular, $\mathcal{P}_{p} \neq \emptyset$. 
We now present the bend-and-cancel argument. Choose a "cubication" of $M$, i.e., a $(n+1)$-dimensional cubical subcomplex $K$ of $I^{m}$ for some $m$, and a Lipschitz homeomorphism $G: K \rightarrow M$ such that $G^{-1}: M \rightarrow K$ is also Lipschitz. For each $k \in \mathbb{N}, K(k)$ denotes the cubical complex one gets by subdividing each cube in $K$ into smaller cubes of side length $3^{-k}$. The set of all $q$-cells of $K(k)$ is denoted by $K(k)_{q}$ and we denote by $c(k) \subset M$ the image under $G$ of the set consisting of the centers of the $(n+1)$-cubes $\sigma \in K(k)_{n+1}$.

Using a retraction from $I^{n+1}$ minus a neighborhood of its center into $\partial I^{n+1}$, one can find a retraction from $M$ minus a neighborhood of $c(k)$ onto the $n$-skeleton $G\left(K(k)_{n}\right)$.

9.5. Proposition. There exist positive constants $C_{1}$ and $\varepsilon_{0}$, depending only on $(M, g)$, so that for all $k \in \mathbb{N}$ and $0<\varepsilon \leq \varepsilon_{0}$ we can find a Lipschitz map $F: M \rightarrow M$ such that

- $F$ is homotopic to the identity;

- $F\left(M \backslash B_{\varepsilon 3^{-k}}(c(k))\right) \subset G\left(K(k)_{n}\right)$;

- $|D F| \leq C_{1} \varepsilon^{-1}$.

The proof can be found in [20, Section 5] or [30, Section 5].

In Lemma 5.3 of $[\mathbf{3 0}]$ we show that the Morse function $f$ can be chosen so that, for every $k \in \mathbb{N}$, each level set $f^{-1}(t) \operatorname{intersects~} c(k)$ in at most one point and no critical point of $f$ belongs to $c(k)$. Thus, we can find $\varepsilon$ sufficiently small so that for all $t \in \mathbb{R}$ and $k \in \mathbb{N}$

$$
\mathbf{M}\left(f^{-1}(t)\left\llcorner B_{\varepsilon 3^{-k}}(c(k))\right) \leq 2 \omega_{n} \varepsilon^{n} 3^{-n k},\right.
$$

where $\omega_{n}$ is the volume of the unit $n$-ball.

Given $p \in \mathbb{N}$ choose a non-negative integer $k$ so that $3^{k} \leq p^{\frac{1}{n+1}} \leq 3^{k+1}$. For a choice of $\varepsilon$ so that the inequality above holds, consider the map $F$ given by Proposition 9.5 and set

$$
\Phi: \mathbb{R P}^{p} \rightarrow \mathcal{Z}_{n}\left(M ; \mathbb{Z}_{2}\right), \quad \Phi(\theta)=F_{\#}\left(\Psi_{p}(\theta)\right) .
$$

Since $F$ is Lipschitz and homotopic to the identity we obtain that $\Phi \in \mathcal{P}_{p}$.

We now estimate $\mathbf{M}(\Phi(\theta))$ for all $\theta \in \mathbb{R P}^{p}$. We have

$$
\begin{aligned}
\mathbf{M}\left(F_{\#}\left(f^{-1}(t)\left\llcorner B_{\varepsilon 3^{-k}}(c(k))\right)\right)\right. & \leq\left(\sup _{M}|D F|\right)^{n} \mathbf{M}\left(f^{-1}(t)\left\llcorner B_{\varepsilon 3^{-k}}(c(k))\right)\right. \\
& \leq 2\left(\sup _{M}|D F|\right)^{n} \omega_{n} \varepsilon^{n} 3^{-n k} \leq 2 C_{1}^{n} \omega_{n} 3^{-n k} .
\end{aligned}
$$

Because each $\Psi_{p}(\theta)$ consists of at most $p$ level surfaces of $f$, we obtain

$$
\mathbf{M}\left(F_{\#}\left(\Psi_{p}(\theta)\left\llcorner B_{\varepsilon 3^{-k}}(c(k))\right)\right) \leq 2 p C_{1}^{n} \omega_{n} 3^{-n k}\right.
$$

for all $\theta \in \mathbb{R P}^{p}$.

Set $B=M \backslash B_{\varepsilon 3^{-k}}(c(k))$. From the second property of Proposition 9.5 we have that the support of $F_{\#}\left(\Psi_{p}(\theta)\llcorner B)\right.$ is contained in the $n$-skeleton 
$G\left(K(k)_{n}\right)$. Since we are using $\mathbb{Z}_{2}$ coefficients the multiplicity is at most one. Hence

$$
\begin{aligned}
\mathbf{M}\left(F_{\#}\left(\Psi_{p}(\theta)\llcorner B)\right) \leq \mathbf{M}(\right. & \left.G\left(K(k)_{n}\right)\right) \\
& \leq 2(n+1) C_{2}\left(\sup _{K}|D G|\right)^{n} 3^{k(n+1)} 3^{-k n}=C_{3} 3^{k},
\end{aligned}
$$

where $C_{2}$ is the number of $(n+1)$-cells in the cell complex $K$ and $C_{3}=$ $2(n+1) C_{2}\left(\sup _{K}|D G|\right)^{n}$ depends only on $M$.

Combining this inequality with $(22)$ and since $3^{k} \leq p^{\frac{1}{n+1}} \leq 3^{k+1}$ we have, for some constant $C=C(M, g)$,

$$
\mathbf{M}(\Phi(\theta)) \leq 2 p C_{1}^{n} \omega_{n} 3^{-n k}+C_{3} 3^{k} \leq C p^{\frac{1}{n+1}} \text { for all } \theta \in \mathbb{R P}^{p} .
$$

Therefore $\omega_{p}(M) \leq C p^{\frac{1}{n+1}}$.

\section{Application: Existence of infinitely many minimal hypersurfaces}

We apply Almgren-Pitts Min-max Theory to the families discussed in the previous section and prove

10.1. Theorem. Let $(M, g)$ be a compact Riemannian manifold of $d i$ mension $(n+1)$, with $2 \leq n \leq 6$, and positive Ricci curvature.

Then $M$ contains an infinite number of distinct smooth, closed, embedded, minimal hypersurfaces.

Before we prove this theorem we need to establish some preliminary results.

Denote by $\mathcal{P}_{p, \mathbf{M}}$ the subset of $\mathcal{P}_{p}$ made by those maps which are continuous in the mass topology. It follows at once from Theorem 2.9 that

$$
\omega_{p}(M)=\inf _{\Phi \in \mathcal{P}_{p, \mathbf{M}}} \sup \{\mathbf{M}(\Phi(x)): x \in \operatorname{dmn}(\Phi)\} .
$$

A priori, it is not clear whether $\omega_{p}(M)$ equals $\mathbf{L}\left([\Phi]_{\mathbf{M}}\right)$ for some $\Phi \in$ $\mathcal{P}_{p, \mathbf{M}}$. The next proposition characterizes the case where that never occurs.

10.2. Proposition. Assume $2 \leq n \leq 6$. If there is $p \in \mathbb{N}$ such that

$$
\omega_{p}(M)<\mathbf{L}\left([\Phi]_{\mathbf{M}}\right) \text { for every } \Phi \in \mathcal{P}_{p, \mathbf{M}},
$$

then there are infinitely many distinct minimal embedded hypersurfaces with area uniformly bounded.

Proof. If $\Phi \in \mathcal{P}_{p, \mathbf{M}}$ we have that every element $\Psi \in[\Phi]_{\mathbf{M}}$ also belongs to $\mathcal{P}_{p, \mathbf{M}}$ and so $\omega_{p}(M) \leq \mathbf{L}\left([\Phi]_{\mathbf{M}}\right)$. Hence, under the assumptions of the proposition, we can find a sequence of $p$-admissible cubical subcomplexes $X_{k}$ and a sequence of $p$-sweepouts $\Phi_{k} \in \mathcal{P}_{p, \mathbf{M}}$ with domain $X_{k}$ such that

$$
\mathbf{L}\left(\left[\Phi_{1}\right]_{\mathbf{M}}\right)>\cdots>\mathbf{L}\left(\left[\Phi_{k}\right]_{\mathbf{M}}\right)>\mathbf{L}\left(\left[\Phi_{k+1}\right]_{\mathbf{M}}\right)>\ldots
$$


and

$$
\lim _{k \rightarrow \infty} \mathbf{L}\left(\left[\Phi_{k}\right]_{\mathbf{M}}\right)=\omega_{p}(M)
$$

Theorem 2.12 implies $\mathbf{L}\left(\left[\Phi_{k}\right]_{\mathbf{M}}\right)=\left\|V_{k}\right\|(M)$ for some smooth closed embedded minimal hypersurface $V_{k}$, possibly disconnected and with integer multiplicities, and thus the proposition follows.

10.3. Lusternik-Schnirelmann theory. We apply Lusternik-Schnirelmann theory to prove:

10.4. Theorem. Assume that $2 \leq n \leq 6$. If $\omega_{p}(M)=\omega_{p+1}(M)$ for some $p \in \mathbb{N}$, then there exist infinitely many distinct smooth, closed, embedded minimal hypersurfaces in $M$.

Sketch of PROOF. We explain the main ideas and refer the reader to [30, Section 6] for details.

By Proposition 10.2, we can assume that there exist a $(\mathrm{p}+1)$-admissible cubical subcomplex $X$ and a $(p+1)$-sweepout $\Phi \in \mathcal{P}_{p, \mathbf{M}}$ so that $\omega_{p+1}(M)=$ $\mathbf{L}\left([\Phi]_{\mathbf{M}}\right)$. Thus, by Theorem 2.5 , we can choose a sequence $\Phi_{i} \in[\Phi]_{\mathbf{M}}$ so that $\sup _{x \in X} \mathbf{M}\left(\Phi_{i}(x)\right)$ tends to $\omega_{p+1}(M)$ and every element in $\mathbf{C}\left(\left\{\Phi_{i}\right\}_{i \in \mathbb{N}}\right)$ is a stationary varifold.

Suppose, by contradiction, that there are only finitely many smooth, closed, embedded minimal hypersurfaces in $M$. Let $\mathcal{T}$ be the set of all $T \in \mathcal{Z}_{n}\left(M ; \mathbb{Z}_{2}\right)$ with $\mathbf{M}(T) \leq w_{p+1}(M)$ and such that either $T=0$ or the support of $T$ is a smooth closed embedded minimal hypersurface. By the contradiction hypothesis, $\mathcal{T}$ is a finite set.

Claim: There is $\varepsilon>0$ so that every continuous map in the flat topology $\Psi: S^{1} \rightarrow \mathcal{Z}_{n}\left(M ; \mathbb{Z}_{2}\right)$ with

$$
\Psi\left(S^{1}\right) \subset B_{\varepsilon}^{\mathcal{F}}(\mathcal{T})=\left\{T \in \mathcal{Z}_{n}\left(M ; \mathbb{Z}_{2}\right): \mathcal{F}(T, \mathcal{T})<\varepsilon\right\}
$$

is homotopically trivial.

If $\varepsilon$ is chosen small enough (depending on $\mathcal{T}$ ), then there must exist $S \in \mathcal{T}$ so that

$$
\Psi\left(S^{1}\right) \subset B_{\varepsilon}^{\mathcal{F}}(S)
$$

In this case, $\Psi$ is homotopic to a constant map by Proposition 12.2 and so $\Psi$ cannot be a sweepout.

With this choice of $\varepsilon$, set

$$
Y_{i}=\left\{x \in X: \mathcal{F}\left(\Phi_{i}(x), \mathcal{T}\right) \geq \varepsilon\right\} .
$$

10.5. Lemma. For all $i$ sufficiently large we have $\left(\Phi_{i}\right)_{\mid Y_{i}} \in \mathcal{P}_{p, \mathbf{M}}$.

Proof. The map $\left(\Phi_{i}\right)_{\mid Y_{i}}$ is continuous in the mass topology and thus we only need to check that it is a $p$-sweepout.

Let $\lambda=\Phi_{i}^{*}(\bar{\lambda}) \in H^{1}\left(X ; \mathbb{Z}_{2}\right)$. Then, since $\Phi_{i}$ is a $(p+1)$-sweepout, we have

- for every curve $\gamma: S^{1} \rightarrow X, \lambda(\gamma) \neq 0$ if and only if $\Phi_{i} \circ \gamma$ is a sweepout; 
- $\lambda^{p+1} \neq 0$ in $H^{p+1}\left(X ; \mathbb{Z}_{2}\right)$.

Let $Z_{i}=X \backslash Y_{i}$. If we show that $\lambda^{p} \neq 0$ in $H^{p}\left(Y_{i} ; \mathbb{Z}_{2}\right)$, it follows at once that $\left(\Phi_{i}\right)_{\mid Y_{i}}$ is a $p$-sweepout.

For any closed curve $\gamma: S^{1} \rightarrow Z_{i}$, we have that

$$
\Phi_{i} \circ \gamma\left(S^{1}\right) \subset B_{\varepsilon}^{\mathcal{F}}(\mathcal{T})
$$

and so $\Phi_{i} \circ \gamma: S^{1} \rightarrow \mathcal{Z}_{n}\left(M ; \mathbb{Z}_{2}\right)$ is not a sweepout. Thus $\lambda(\gamma)=0$ and this means $\lambda=0$ in $H^{1}\left(Z_{i} ; \mathbb{Z}_{2}\right)$ because $H^{1}\left(Z_{i} ; \mathbb{Z}_{2}\right)=\operatorname{Hom}\left(H_{1}\left(Z_{i}\right) ; \mathbb{Z}_{2}\right)$, by the Universal Coefficient Theorem.

From the natural exact sequence

$$
H^{1}\left(X, Z_{i} ; \mathbb{Z}_{2}\right) \stackrel{j^{*}}{\rightarrow} H^{1}\left(X ; \mathbb{Z}_{2}\right) \stackrel{i^{*}}{\rightarrow} H^{1}\left(Z_{i} ; \mathbb{Z}_{2}\right)
$$

we obtain that $\lambda=j^{*} \lambda_{1}$ for some $\lambda_{1} \in H^{1}\left(X, Z_{i} ; \mathbb{Z}_{2}\right)$.

Suppose $\lambda^{p}=0$ in $H^{p}\left(Y_{i} ; \mathbb{Z}_{2}\right)$. Then the exact sequence

$$
H^{p}\left(X, Y_{i} ; \mathbb{Z}_{2}\right) \stackrel{j^{*}}{\rightarrow} H^{p}\left(X ; \mathbb{Z}_{2}\right) \stackrel{i^{*}}{\rightarrow} H^{p}\left(Y_{i} ; \mathbb{Z}_{2}\right)
$$

implies that $j^{*} \lambda_{2}=\lambda^{p}$ for some $\lambda_{2} \in H^{p}\left(X, Y_{i} ; \mathbb{Z}_{2}\right)$.

Thus

$$
j^{*} \lambda_{1} \smile j^{*} \lambda_{2}=\lambda^{p+1} \neq 0 \text { in } H^{p+1}\left(X ; \mathbb{Z}_{2}\right) .
$$

On the other hand, from the relative cup product (see [21], p 209):

$$
H^{1}\left(X, Z_{i} ; \mathbb{Z}_{2}\right) \smile H^{p}\left(X, Y_{i} ; \mathbb{Z}_{2}\right) \rightarrow H^{p+1}\left(X, Y_{i} \cup Z_{i} ; \mathbb{Z}_{2}\right) .
$$

But $Y_{i} \cup Z_{i}=X$, hence $H^{p+1}\left(X, Y_{i} \cup Z_{i} ; \mathbb{Z}_{2}\right)=H^{p+1}\left(X, X ; \mathbb{Z}_{2}\right)=0$. In particular, $\lambda_{1} \smile \lambda_{2}=0$. This is a contradiction because

$$
j^{*}\left(\lambda_{1} \smile \lambda_{2}\right)=j^{*} \lambda_{1} \smile j^{*} \lambda_{2}=\lambda^{p+1} \neq 0 .
$$

Hence $\lambda^{p} \neq 0$ in $H^{p}\left(Y_{i} ; \mathbb{Z}_{2}\right)$ and the proof is finished.

Set

$$
L=\limsup _{i \rightarrow \infty} \max \left\{\mathbf{M}\left(\Phi_{i}(y)\right): y \in Y_{i}\right\} .
$$

Naturally, $L \leq \omega_{p+1}(M)$ and the previous lemma implies $L \geq \omega_{p}(M)=$ $\omega_{p+1}(M)$, which means that equality holds.

We now describe the idea to exclude the case $L=\omega_{p}(M)$ and obtain a contradiction. The argument in [30, Section 6] requires the use of the discrete theory of Almgren-Pitts and the reader can see there the details.

Consider the set

$$
\begin{aligned}
\mathbf{C}=\{V:\|V\|(M) & =L, V=\lim _{j \rightarrow \infty}\left|\Phi_{i_{j}}\left(y_{j}\right)\right| \text { as varifolds, } \\
& \text { for some increasing sequence } \left.\left\{i_{j}\right\}_{j \in \mathbb{N}} \text { and } y_{j} \in Y_{i_{j}}\right\} .
\end{aligned}
$$

Every element in $\mathbf{C}$ is a stationary varifold because $\mathbf{C} \subset \mathbf{C}\left(\left\{\Phi_{i}\right\}_{i \in \mathbb{N}}\right)$. 
10.6. Lemma. No $V \in \mathbf{C}$ has smooth, closed, embedded support.

Proof. Suppose the claim is false for some $V \in \mathbf{C}$. This implies that spt $\|V\|$ is a smooth, closed, embedded minimal hypersurface which belongs to $\mathcal{T}$ because $\mathbf{M}(V) \leq \omega_{p+1}(M)$.

From the definition of the set $Y_{i}$ we see that we can find a sequence $\left\{T_{k}\right\} \subset \mathcal{Z}_{n}\left(M ; \mathbb{Z}_{2}\right)$ with $\mathbf{F}\left(\left|T_{k}\right|, V\right)<1 / k$ and $\mathcal{F}\left(T_{k}, \mathcal{T}\right) \geq \varepsilon$ for every $k$. By compactness, there exists a subsequence $\left\{T_{l}\right\} \subset\left\{T_{k}\right\}$ that converges in the flat topology to some $T \in \mathcal{Z}_{n}\left(M ; \mathbb{Z}_{2}\right)$ and whose associated sequence of varifolds $\left\{\left|T_{l}\right|\right\}$ converges in varifold topology to $V$. In particular, $\mathcal{F}(T, \mathcal{T}) \geq$ $\varepsilon$ and $\mathbf{M}(T) \leq \omega_{p+1}(M)$. We also have, by lower semicontinuity of mass, that

$$
\mathbf{M}(T\llcorner(M \backslash \operatorname{spt}\|V\|))=0 .
$$

This implies that the support of $T$ is contained in spt $\|V\|$ and so, by the Constancy Theorem $([\mathbf{4 1}]), T \in \mathcal{T}$. This contradicts $\mathcal{F}(T, \mathcal{T}) \geq \varepsilon$.

The above lemma implies that no element in $\mathbf{C}$ is $\mathbb{Z}_{2}$ almost minimizing in annuli (see Definition 2.10 of $[\mathbf{3 0}]$ ) and so the combinatorial Theorem of Pitts $\left[\mathbf{3 7}\right.$, Theorem 4.10] produces $\Psi_{i}^{*} \in\left[\left(\Phi_{i}\right)_{\mid Y_{i}}\right]_{\mathbf{M}}$ with

$$
\sup _{y \in Y_{i}} \mathbf{M}\left(\Psi_{i}^{*}(y)\right)<L=\omega_{p+1}(M)=\omega_{p}(M)
$$

for all large $i$. This is a contradiction because, by Lemma 10.5, $\Psi_{i}^{*} \in \mathcal{P}_{p, \mathbf{M}}$ and so $\omega_{p}(M) \leq \sup _{y \in Y_{i}} \mathbf{M}\left(\Psi_{i}^{*}(y)\right)$.

Therefore there must be infinitely many distinct smooth, closed, embedded minimal hypersurfaces in $M$.

10.7. Proof of Theorem 10.1. The proof is by contradiction. Suppose that the set $\mathcal{L}$ of all smooth, closed, embedded minimal hypersurfaces of $M$ is finite.

It follows from Proposition 10.2 that for every $p \geq 1$ we can find $p$ admissible $X_{p}$ and $\Phi_{p} \in \mathcal{P}_{p, \mathbf{M}}$ with domain $X_{p}$ so that

$$
\omega_{p}(M)=\mathbf{L}\left(\left[\Phi_{p}\right]_{\mathbf{M}}\right) .
$$

By Theorem 2.12 we have

$$
\omega_{p}(M)=\left\|V_{p}\right\|(M)
$$

for some $V_{p} \in \mathcal{I} \mathcal{V}_{n}(M)$, where $V_{p}$ is the varifold of a smooth, closed, embedded minimal hypersurface, with possible multiplicities.

Since the support of $V_{p}$ is embedded and the ambient manifold has positive Ricci curvature, Frankel's Theorem [13] implies that the support of $V_{p}$ is connected. Hence $V_{p}=n_{p} \Sigma_{p}$ for some $\Sigma_{p} \in \mathcal{L}$ and $n_{p} \in \mathbb{N}$, for all $p \in \mathbb{N}$. And since we are assuming that $\mathcal{L}$ is finite, we must have by Theorem 10.4 that

$$
\left\|V_{p}\right\|(M)<\left\|V_{p+1}\right\|(M) \text { for all } p \in \mathbb{N} \text {. }
$$


Combining this with the fact that

$$
n_{p}\left|\Sigma_{p}\right|=|| V_{p} \|(M)=\omega_{p}(M) \leq C p^{\frac{1}{n+1}} \quad \text { for all } p \in \mathbb{N},
$$

by Theorem 9.3, we have

$$
\begin{aligned}
\#\left\{a=k|\Sigma|: k \in \mathbb{N}, \Sigma \in \mathcal{L}, k|\Sigma| \leq C p^{\frac{1}{n+1}}\right\} & \\
& \geq \#\left\{\omega_{k}(M): k=1, \ldots, p\right\}=p .
\end{aligned}
$$

But if $N$ denotes the number of elements of $\mathcal{L}$, and $\delta>0$ is such that $|\Sigma| \geq \delta$ for every $\Sigma \in \mathcal{L}$, then we also have

$$
\#\left\{a=k|\Sigma|: k \in \mathbb{N}, \Sigma \in \mathcal{L}, k|\Sigma| \leq C p^{\frac{1}{n+1}}\right\} \leq \frac{1}{\delta} C N p^{\frac{1}{n+1}} .
$$

We get a contradiction for sufficiently large $p$. Hence $\mathcal{L}$ is infinite.

\section{Almgren-Pitts Min-max theory II}

We set up the Almgren-Pitts Min-max theory following closely [37].

Let $X$ be a cubical subcomplex of the $m$-dimensional cube $I^{m}=[0,1]^{m}$. Each $k$-cell of $I^{m}$ is of the form $\alpha_{1} \otimes \cdots \otimes \alpha_{m}$, where $\alpha_{i} \in\{0,1,[0,1]\}$ for every $i$ and $\sum_{i=1}^{m} \operatorname{dim}\left(\alpha_{i}\right)=k$. Notice that every polyhedron is homeomorphic to the support of some cubical subcomplex of this type [9, Chapter 4].

The group $G$ is either $\mathbb{Z}$ or $\mathbb{Z}_{2}$. When the group is omitted from the notation it means $G=\mathbb{Z}$.

Finally, $\Phi_{0}: \partial I^{m} \rightarrow \mathcal{Z}_{n}(M ; \mathbf{F})$ is a continuous map in the $\mathbf{F}$-metric.

11.1. Discrete setting. For each $j \in \mathbb{N}, I(1, j)$ denotes the cube complex on $I^{1}$ whose 1 -cells and 0 -cells (those are sometimes called vertices) are, respectively,

$$
\left[0,3^{-j}\right],\left[3^{-j}, 2 \cdot 3^{-j}\right], \ldots,\left[1-3^{-j}, 1\right] \quad \text { and } \quad[0],\left[3^{-j}\right], \ldots,\left[1-3^{-j}\right],[1] .
$$

We denote by $I(m, j)$ the cell complex on $I^{m}$ :

$$
I(m, j)=I(1, j) \otimes \ldots \otimes I(1, j) \quad(m \text { times }) .
$$

Then $\alpha=\alpha_{1} \otimes \cdots \otimes \alpha_{m}$ is a $q$-cell of $I(m, j)$ if and only if $\alpha_{i}$ is a cell of $I(1, j)$ for each $i$, and $\sum_{i=1}^{m} \operatorname{dim}\left(\alpha_{i}\right)=q$. We often abuse notation by identifying a $q$-cell $\alpha$ with its support: $\alpha_{1} \times \cdots \times \alpha_{m} \subset I^{m}$.

The cube complex $X(j)$ is the union of all cells of $I(m, j)$ whose support is contained in some cell of $X$. We use the notation $X(j)_{q}$ to denote the set of all $q$-cells in $X(j)$. Two vertices $x, y \in X(j)_{0}$ are adjacent if they belong to a common cell in $X(j)_{1}$.

For the relative version, we also need to consider $I_{0}(n, j)$ the subcomplex of $I(n, j)$ generated by all cells whose support is contained in $\partial I^{n}$ and $I_{0}(n, j)_{p}$ denotes the set of all $p$-cells of $I(n, j)$ whose support is contained in $\partial I^{n}$;

Given $i, j \in \mathbb{N}$ we define $\mathbf{n}(i, j): X(i)_{0} \rightarrow X(j)_{0}$ so that $\mathbf{n}(i, j)(x)$ is the element in $X(j)_{0}$ that is closest to $x$ (see [37, page 141] or [31, Section 7.1] for a precise definition). 
Given a map $\phi: X(j)_{0} \rightarrow \mathcal{Z}_{n}(M ; G)$, we define the fineness of $\phi$ to be

$$
\mathbf{f}(\phi)=\sup \left\{\mathbf{M}(\phi(x)-\phi(y)): x, y \text { adjacent vertices in } X(j)_{0}\right\} \text {. }
$$

The reader should think of the notion of fineness as being a discrete measure of continuity with respect to the mass norm.

11.2. Homotopy notions. Let $\phi_{i}: X\left(k_{i}\right)_{0} \rightarrow \mathcal{Z}_{n}(M ; G), i=1,2$. We say that $\phi_{1}$ is $X$-homotopic to $\phi_{2}$ in $\mathcal{Z}_{n}(M ; \mathbf{M} ; G)$ with fineness $\delta$ if we can find $k \in \mathbb{N}$ and a map

$$
\psi: I(1, k)_{0} \times X(k)_{0} \rightarrow \mathcal{Z}_{n}(M ; G)
$$

such that

(i) $\mathbf{f}(\psi)<\delta$;

(ii) if $i=1,2$ and $x \in X(k)_{0}$, then

$$
\psi([i-1], x)=\phi_{i}\left(\mathbf{n}\left(k, k_{i}\right)(x)\right) .
$$

Instead of considering continuous maps from $X$ into $\mathcal{Z}_{n}(M ; \mathbf{M} ; G)$, the Almgren-Pitts theory deals with sequences of discrete maps into $\mathcal{Z}_{n}(M ; G)$ with finenesses tending to zero.

\subsection{Definition. A}

$(X, \mathbf{M})$-homotopy sequence of mappings into $\mathcal{Z}_{n}(M ; \mathbf{M} ; G)$

is a sequence of mappings $S=\left\{\phi_{i}\right\}_{i \in \mathbb{N}}$,

$$
\phi_{i}: X\left(k_{i}\right)_{0} \rightarrow \mathcal{Z}_{n}(M ; G),
$$

such that $\phi_{i}$ is $X$-homotopic to $\phi_{i+1}$ in $\mathcal{Z}_{n}(M ; \mathbf{M} ; G)$ with fineness $\delta_{i}$ and

(i) $\lim _{i \rightarrow \infty} \delta_{i}=0$;

(ii) $\sup \left\{\mathbf{M}\left(\phi_{i}(x)\right): x \in X\left(k_{i}\right)_{0}, i \in \mathbb{N}\right\}<+\infty$.

The next definition explains what it means for two distinct homotopy sequences of mappings into $\mathcal{Z}_{n}(M ; \mathbf{M} ; G)$ to be homotopic.

11.4. Definition. Let $S^{1}=\left\{\phi_{i}^{1}\right\}_{i \in \mathbb{N}}$ and $S^{2}=\left\{\phi_{i}^{2}\right\}_{i \in \mathbb{N}}$ be $(X, \mathbf{M})$ homotopy sequences of mappings into $\mathcal{Z}_{n}(M ; \mathbf{M} ; G)$. We say that $S^{1}$ is homotopic with $S^{2}$ if there exists a sequence $\left\{\delta_{i}\right\}_{i \in \mathbb{N}}$ such that

- $\phi_{i}^{1}$ is $X$-homotopic to $\phi_{i}^{2}$ in $\mathcal{Z}_{n}(M ; \mathbf{M} ; G)$ with fineness $\delta_{i}$;

- $\lim _{i \rightarrow \infty} \delta_{i}=0$.

The relation "is homotopic with" is an equivalence relation on the set of all $(X, \mathbf{M})$-homotopy sequences of mappings into $\mathcal{Z}_{n}(M ; \mathbf{M} ; G)$. We call the equivalence class of any such sequence a $(X, \mathbf{M})$-homotopy class of sequence of mappings into $\mathcal{Z}_{n}(M ; \mathbf{M} ; G)$. We denote by $\left[X, \mathcal{Z}_{n}(M ; \mathbf{M} ; G)\right]^{\#}$ the set of all equivalence classes.

11.5. Homotopy notions - Relative version. Recall that $\Phi_{0}: \partial I^{m} \rightarrow$ $\mathcal{Z}_{n}(M ; \mathbf{F})$ is a continuous function in the $\mathbf{F}$-metric. 
11.6. Definition. Let $\phi_{i}: I\left(m, k_{i}\right)_{0} \rightarrow \mathcal{Z}_{n}(M), i=1,2$. We say that $\phi_{1}$ is $m$-homotopic to $\phi_{2}$ in $\left(\mathcal{Z}_{n}(M ; \mathbf{M}), \Phi_{0}\right)$ with fineness $\delta$ if we can find $k \in \mathbb{N}$ and a map

$$
\psi: I(1, k)_{0} \times I(m, k)_{0} \rightarrow \mathcal{Z}_{n}(M)
$$

such that

(i) $\mathbf{f}(\psi)<\delta$;

(ii) if $i=1,2$ and $x \in I(m, k)_{0}$, then

$$
\psi([i-1], x)=\phi_{i}\left(\mathbf{n}\left(k, k_{i}\right)(x)\right)
$$

(iii)

$$
\begin{aligned}
& \sup \left\{\mathcal{F}\left(\psi(t, x)-\Phi_{0}(x)\right):(t, x) \in I(1, k)_{0} \times I_{0}(m, k)_{0}\right\} \leq \delta, \\
& \mathbf{M}(\psi(t, x)) \leq \mathbf{M}\left(\Phi_{0}(x)\right)+\delta \text { for any }(t, x) \in I(1, k)_{0} \times I_{0}(m, k)_{0} .
\end{aligned}
$$

In particular we must have

$$
\sup \left\{\mathcal{F}\left(\phi_{i}(x)-\Phi_{0}(x)\right): x \in I_{0}\left(m+1, k_{i}\right)_{0}\right\} \leq \delta,
$$

and

$$
\sup \left\{\mathbf{M}\left(\phi_{i}(x)\right)-\mathbf{M}\left(\Phi_{0}(x)\right): x \in I_{0}\left(m+1, k_{i}\right)_{0}\right\} \leq \delta,
$$

for each $i=1,2$.

\subsection{Definition. A}

$\left(I^{m}, \mathbf{M}\right)$-homotopy sequence of mappings into $\left(\mathcal{Z}_{n}(M ; \mathbf{M}), \Phi_{0}\right)$

is a sequence of mappings $\left\{\phi_{i}\right\}_{i \in \mathbb{N}}$,

$$
\phi_{i}: I\left(m, k_{i}\right)_{0} \rightarrow \mathcal{Z}_{n}(M),
$$

such that $\phi_{i}$ is $m$-homotopic to $\phi_{i+1}$ in $\left(\mathcal{Z}_{n}(M ; \mathbf{M}), \Phi_{0}\right)$ with fineness $\delta_{i}$ and

(i) $\lim _{i \rightarrow \infty} \delta_{i}=0$;

(ii) $\sup \left\{\mathbf{M}\left(\phi_{i}(x)\right): x \in I\left(m, k_{i}\right)_{0}, i \in \mathbb{N}\right\}<+\infty$.

The next lemma says that $\phi_{i}$ restricted to the boundary of its domain tends to $\Phi_{0}$ in the $\mathbf{F}$-metric.

11.8. Lemma. Let $S=\left\{\phi_{i}\right\}_{i \in \mathbb{N}}$ be a $\left(I^{m}, \mathbf{M}\right)$-homotopy sequence of mappings into $\left(\mathcal{Z}_{n}(M ; \mathbf{M}), \Phi_{0}\right)$. If $I\left(m, k_{i}\right)_{0}$ denotes the domain of $\phi_{i}$, then

$$
\lim _{i \rightarrow \infty} \sup \left\{\mathbf{F}\left(\phi_{i}(x), \Phi_{0}(x)\right): x \in I_{0}\left(m, k_{i}\right)_{0}\right\}=0 .
$$

Proof. Since $\Phi_{0}$ is continuous in the $\mathbf{F}$-metric, $\Phi_{0}\left(\partial I^{m}\right)$ is a compact subset of $\mathcal{Z}_{n}(M ; \mathbf{F})$. The lemma follows from condition (iii) in Definition 11.6 and Lemma 2.2.

The notion of $S^{1}$ and $S^{2}$ two distinct $\left(I^{m}, \mathbf{M}\right)$-homotopy sequences of mappings into $\left(\mathcal{Z}_{n}(M ; \mathbf{M}), \Phi_{0}\right)$ to be homotopic is given below 
11.9. Definition. Let $S^{1}=\left\{\phi_{i}^{1}\right\}_{i \in \mathbb{N}}$ and $S^{2}=\left\{\phi_{i}^{2}\right\}_{i \in \mathbb{N}}$ be $\left(I^{m}, \mathbf{M}\right)$ homotopy sequences of mappings into $\left(\mathcal{Z}_{n}(M ; \mathbf{M}), \Phi_{0}\right)$. We say that $S^{1}$ is homotopic with $S^{2}$ if there exists a sequence $\left\{\delta_{i}\right\}_{i \in \mathbb{N}}$ such that

- $\phi_{i}^{1}$ is $m$-homotopic to $\phi_{i}^{2}$ in $\mathcal{Z}_{n}(M ; \mathbf{M})$ with fineness $\delta_{i}$;

- $\lim _{i \rightarrow \infty} \delta_{i}=0$.

The relation "is homotopic with" is an equivalence relation on the set of all $(m, \mathbf{M})$-homotopy sequences of mappings into $\left(\mathcal{Z}_{n}(M ; \mathbf{M}), \Phi_{0}\right)$. We call the equivalence class of any such sequence a $\left(I^{m}, \mathbf{M}\right)$-homotopy class of sequence of mappings into $\left(\mathcal{Z}_{n}(M ; \mathbf{M}), \Phi_{0}\right)$. We denote by $\pi_{m}^{\#}\left(\mathcal{Z}_{n}(M ; \mathbf{M}), \Phi_{0}\right)$ the set of all equivalence classes.

11.10. Min-max definitions. Given $\Pi$ in either

$$
\left[X, \mathcal{Z}_{n}(M ; \mathbf{M} ; G)\right]^{\#} \quad \text { or } \quad \pi_{m}^{\#}\left(\mathcal{Z}_{n}(M ; \mathbf{M}), \Phi_{0}\right),
$$

let $\mathbf{L}: \Pi \rightarrow[0,+\infty]$ be defined by

$$
\mathbf{L}(S)=\limsup _{i \rightarrow \infty} \max \left\{\mathbf{M}\left(\phi_{i}(x)\right): x \in \operatorname{dmn}\left(\phi_{i}\right)\right\}, \quad \text { where } S=\left\{\phi_{i}\right\}_{i \in \mathbb{N}} .
$$

Note that $\mathbf{L}(S)$ is the discrete replacement for the maximum area of a continuous map into $\mathcal{Z}_{n}(M ; \mathbf{M} ; G)$.

Given $S=\left\{\phi_{i}\right\}_{i \in \mathbb{N}} \in \Pi$, we also consider the compact subset $\mathbf{K}(S)$ of $\mathcal{V}_{n}(M)$ given by

$$
\begin{aligned}
& \mathbf{K}(S)=\left\{V: V=\lim _{j \rightarrow \infty}\left|\phi_{i_{j}}\left(x_{j}\right)\right|\right. \text { as varifolds, for some increasing } \\
& \text { sequence } \left.\left\{i_{j}\right\}_{j \in \mathbb{N}} \text { and } x_{j} \in \operatorname{dmn}\left(\phi_{i_{j}}\right)\right\} .
\end{aligned}
$$

This is the discrete replacement for the image of a continuous map into $\mathcal{Z}_{n}(M ; \mathbf{M} ; G)$.

11.11. Definition. The width of $\Pi$ is defined by

$$
\mathbf{L}(\Pi)=\inf \{\mathbf{L}(S): S \in \Pi\} .
$$

We say $S \in \Pi$ is a critical sequence for $\Pi$ if

$$
\mathbf{L}(S)=\mathbf{L}(\Pi) .
$$

The critical set $\mathbf{C}(S)$ of a critical sequence $S \in \Pi$ is given by

$$
\mathbf{C}(S)=\mathbf{K}(S) \cap\{V:\|V\|(M)=\mathbf{L}(S)\} .
$$

11.12. Proposition. There exists a critical sequence $S^{*} \in \Pi$. For each critical sequence $S^{*}$, there exists a critical sequence $S \in \Pi$ such that

- $\mathbf{C}(S) \subset \mathbf{C}\left(S^{*}\right)$;

- every $\Sigma \in \mathbf{C}(S)$ is either a stationary varifold or, if we are in the relative case, every $\Sigma \in \mathbf{C}(S)$ is either a stationary varifold or belongs to $\left|\Phi_{0}\right|\left(\partial I^{m}\right)$.

The proof consists of a pull-tight argument and can be seen in Theorem 4.3 of [37] (see also Section 15 of [31]). 
11.13. Min-max Theorem. We now adapt the celebrated Pitts MinMax Theorem to our setting.

11.14. Theorem. Assume $2 \leq n \leq 6$. Consider $\Pi$ in either

$$
\left[X, \mathcal{Z}_{n}(M ; \mathbf{M} ; G)\right]^{\#} \quad \text { or } \quad \pi_{m}^{\#}\left(\mathcal{Z}_{n}(M ; \mathbf{M}), \Phi_{0}\right)
$$

and assume, respectively, that

$$
\mathbf{L}(\Pi)>0 \quad \text { or } \quad \mathbf{L}(\Pi)>\sup \left\{\mathbf{M}\left(\Phi_{0}(x)\right): x \in \partial I^{m}\right\} .
$$

Then there exists a stationary integral rectifiable varifold $\Sigma$ such that

(1) $\|\Sigma\|(M)=\mathbf{L}(\Pi)$;

(2) the support of $\Sigma$ is a smooth compact embedded minimal hypersurface.

Moreover,

- if $S^{*}$ is a critical sequence for $\Pi$ then $\Sigma$ can be chosen so that $\Sigma \in \mathbf{C}\left(S^{*}\right)$;

- we can choose a critical sequence $S$ so that every element in $\mathbf{C}(S)$ is a stationary varifold.

Proof. We consider first the case where $\Pi \in\left[X, \mathcal{Z}_{n}(M ; \mathbf{M} ; G)\right]^{\#}$.

In Theorem 4.10, Pitts showed that given a critical sequence $S^{*} \in \Pi$ there exists a stationary varifold $\Sigma$ satisfying (1) which is $G$ almost minimizing in annuli (see [37, Section 3.1] for the definition). He showed this when $X=I^{m}$ but the argument extends immediately when $X$ is a cubical subcomplex.

When $G=\mathbb{Z}$, Schoen and Simon showed that the support of $\Sigma$ is a compact embedded minimal hypersurface, which is smooth outside a set of Hausdorff codimension 7. Hence, when $2 \leq n \leq 6$, the support of $\Sigma$ is smooth. This regularity result was also shown by Pitts [37] for $2 \leq n \leq 5$.

When $G=\mathbb{Z}_{2}$, Schoen and Simon arguments also give that the support of $\Sigma$ is a smooth compact embedded minimal hypersurface when $2 \leq n \leq 6$ and this is explained in Theorem 2.11 of $[\mathbf{3 2}]$.

We consider now the case where $\Pi \in \pi_{m}^{\#}\left(\mathcal{Z}_{n}(M ; \mathbf{M}), \Phi_{0}\right)$.

Choose a critical sequence $S^{*}$, consider $S=\left\{\varphi_{i}\right\}_{i \in \mathbb{N}} \in \Pi$ given by Proposition 11.12, and let

$$
0<\varepsilon=\mathbf{L}(S)-\max \left\{\mathbf{M}\left(\Phi_{0}(x)\right): x \in \partial I^{m}\right\} .
$$

Because every $\Sigma \in \mathbf{C}(S)$ satisfies

$$
\|\Sigma\|(M)=\mathbf{L}(\Pi)>\max \left\{\mathbf{M}\left(\Phi_{0}(x)\right): x \in \partial I^{m}\right\},
$$

we obtain that every $\Sigma$ in $\mathbf{C}(S)$ must be stationary. Since the construction of $\left[\mathbf{3 7}\right.$, Theorem 4.10] can be made to not affect those $\varphi_{i}(x)$ with

$$
\mathbf{M}\left(\varphi_{i}(x)\right) \leq \mathbf{L}(S)-\varepsilon / 2,
$$

and since

$$
\mathbf{M}\left(\varphi_{i}(x)\right) \leq \max \left\{\mathbf{M}(\Phi(x)): x \in \partial I^{m}\right\}+\varepsilon / 2
$$


for every $x \in \operatorname{dmn}\left(\varphi_{i}\right) \cap \partial I^{m}$ and sufficiently large $i$, we obtain that the competitor $\left\{\varphi_{i}^{*}\right\}_{i \in \mathbb{N}}$ constructed by Pitts belongs to $\Pi$. Therefore, as in [37], we can find an almost-minimizing (in annular regions) $\Sigma \in \mathbf{C}(S)$. The regularity theory we mentioned in the previous case implies that $\Sigma$ is an integral varifold whose support is a smooth embedded minimal surface.

11.15. Interpolation from discrete to continuous. In order to deduce Theorem 2.5 and Theorem 2.12 from Theorem 11.14 we need to to be able to construct a continuous map in the mass topology out of a discrete map with small fineness.

The next theorem follows from Theorem 14.1 in [31].

11.16. Theorem. There exist positive constants $C_{0}=C_{0}(M, m)$ and $\delta_{0}=\delta_{0}(M, m)$ so that if $Y$ is a cubical subcomplex of $I(m, k)$ and

$$
\phi: Y_{0} \rightarrow \mathcal{Z}_{n}(M ; G)
$$

has $\mathbf{f}(\phi)<\delta_{0}$, then there exists a map

$$
\Phi: Y \rightarrow \mathcal{Z}_{n}(M ; \mathbf{M} ; G)
$$

continuous in the mass norm and satisfying

(i) $\Phi(x)=\phi(x)$ for all $x \in Y_{0}$;

(ii) if $\alpha$ is some $j$-cell in $Y_{j}$, then $\Phi$ restricted to $\alpha$ depends only on the values of $\phi$ assumed on the vertices of $\alpha$;

(iii)

$$
\sup \{\mathbf{M}(\Phi(x)-\Phi(y)): x, y \text { lie in a common cell of } Y\} \leq C_{0} \mathbf{f}(\phi) \text {. }
$$

We call $\Phi$ given by Theorem 11.16 the Almgren extension of $\phi$.

11.17. Interpolation from continuous to discrete. Recall the definition of $\mathcal{E}\left(\Phi_{0}\right)$ in Section 2.4.

The next theorem follows from Theorem 13.1 in [31]. There we used $X=I^{m}$ and $G=\mathbb{Z}$ but the proof adapts with no modifications. This result is essential to prove Theorem 2.9 and Theorem 2.13.

11.18. Theorem. Let $\Phi: X \rightarrow \mathcal{Z}_{n}(M ; G)$ be a continuous map in the flat topology with no concentration of mass. There exist a sequence of maps

$$
\phi_{i}: X\left(k_{i}\right)_{0} \rightarrow \mathcal{Z}_{n}(M),
$$

with $k_{i}<k_{i+1}$, and a sequence of positive numbers $\left\{\delta_{i}\right\}_{i \in \mathbb{N}}$ tending to zero such that

(i) $S=\left\{\phi_{i}\right\}$ is a $(X, \mathbf{M})$-homotopy sequence of mappings into $\mathcal{Z}_{n}(M ; \mathbf{M} ; G)$ with $\mathbf{f}\left(\psi_{i}\right)<\delta_{i}$.

(ii)

$$
\max \left\{\mathbf{M}\left(\phi_{i}(x)\right): x \in X\left(k_{i}\right)_{0}\right\} \leq \sup \{\mathbf{M}(\Phi(x)): x \in X\}+\delta_{i} ;
$$

(iii)

$$
\sup \left\{\mathcal{F}\left(\phi_{i}(x)-\Phi(x)\right): x \in X\left(k_{i}\right)_{0}\right\} \leq \delta_{i}
$$


If $X=I^{m}$ and $\Phi \in \mathcal{E}\left(\Phi_{0}\right)$, then $S$ is a $\left(I^{m}, \mathbf{M}\right)$-homotopy sequence of mappings into $\left(\mathcal{Z}_{n}(M ; \mathbf{M}), \Phi_{0}\right)$ with $\mathbf{f}\left(\psi_{i}\right)<\delta_{i}$.

\section{Proof of Min-Max Theorems of Section 2}

We now prove Theorem 2.5, Theorem 2.9, Theorem 2.12, and Theorem 2.13 .

In order to do this, we need to show that continuous maps in the mass or flat topology which are close to each other are homotopic in the mass or flat topology, respectively.

12.1. Proposition. Fix $m \in \mathbb{N}$ and let $\nu$ denote either the mass or flat metric.

There are positive real numbers $\gamma_{m}$ and $\mu_{m}$, depending only on $M$ and $m$, with the following property:

For every continuous map in the $\nu$ topology $\Phi: I^{k} \rightarrow \mathcal{Z}_{n}(M ; \nu ; G)$ with

$$
\Phi\left(I^{k}\right) \subset \mathbf{B}_{\gamma_{m}}^{\nu}(0) \quad \text { and } \Phi(x)=0 \text { for all } x \in \partial I^{k}, \quad k \leq m,
$$

there exists a homotopy $H: I^{k+1} \rightarrow \mathcal{Z}_{n}(M ; \nu ; G)$ with the following properties:

- $H$ is continuous in the $\nu$ topology;

- $H(0, x)=0$ and $H(1, x)=\Phi(x)$ for every $x \in I^{k}$;

- $H(t, x)=0$ for every $x \in \partial I^{k}$ and $t \in[0,1]$;

- $\sup \left\{\nu(H(w)): w \in I^{k+1}\right\} \leq \mu_{m} \sup \left\{\nu(\Phi(x)): x \in I^{k}\right\}$.

Proof. The result follows, as we shall see, from [2, Theorem 6.6] when $\nu$ is the flat metric and from Theorem 11.16 when $\nu=\mathbf{M}$. The arguments are identical and so we prove only the latter case.

Set $\gamma_{m}=\delta_{0} /\left(2\left(C_{0}+1\right)\right)$ and $\mu_{m}=\left(1+2 C_{0}\right)^{2}$, where $\delta_{0}$ and $C_{0}$ are given by Theorem 11.16 .

Define $a_{i}: I(1, i)_{0} \times I(k, i)_{0} \rightarrow \mathcal{Z}_{n}(M ; G)$ as

$$
a_{1}([0], x)=0, \quad a_{i}([0], x)=\Phi(\mathbf{n}(i, i-1)(x)) \quad \text { if } i>1,
$$

and

$$
a_{i}(t, x)=\Phi(x) \text { if } t \neq[0] .
$$

We have $\mathbf{f}\left(a_{i}\right)<2 \gamma_{m}<\delta_{0}$ for all $i \geq 1$ and so we can apply Theorem 11.16 to obtain an Almgren extension $A_{i}$ of $a_{i}$. We have $A_{i}\left(I \times \partial I^{k}\right)=\{0\}$ for all $i \geq 1$ and $A_{1}\left(\{0\} \times I^{k}\right)=\{0\}$.

Consider $b_{i}: I(1, i+1)_{0} \times I(k, i+1)_{0} \rightarrow \mathcal{Z}_{n}(M ; G)$ given by

$$
b_{i}([0], x)=A_{i}(1, x) \quad \text { and } \quad b_{i}(t, x)=\Phi(\mathbf{n}(i+i, i)(x)) \quad \text { if } t \neq[0] .
$$

We have $\mathbf{f}\left(b_{i}\right) \leq C_{0} \mathbf{f}\left(a_{i}\right)+\mathbf{f}\left(a_{i+1}\right)<\delta_{0}$ and so we can apply Theorem 11.16 to obtain an Almgren extension $B_{i}$ of $b_{i}$. From Theorem 11.16 (ii) we obtain that $B_{i}\left(I \times \partial I^{k}\right)=\{0\}$ for all $i \geq 1, B_{i}(0, x)=A_{i}(1, x)$ and $B_{i}(1, x)=A_{i+1}(0, x)$ for all $x \in I^{k}$. 
For each $i \in \mathbb{N}$ set $t_{i}=1-1 /(2 i-1), q_{i}=1-1 /(2 i)$ and define $H:[0,1] \times I^{k} \rightarrow \mathcal{Z}_{n}(M ; G)$ as

$$
H(t, x)=\left\{\begin{aligned}
A_{i}\left(\left(q_{i}-t_{i}\right)^{-1}\left(t-t_{i}\right), x\right) & \text { if } t_{i} \leq t<q_{i} \\
B_{i}\left(\left(t_{i+1}-q_{i}\right)^{-1}\left(t-q_{i}\right), x\right) & \text { if } q_{i} \leq t<t_{i+1} \\
\Phi(x) & \text { if } t=1
\end{aligned}\right.
$$

We leave to the reader to check that $H$ has all the required properties.

The previous proposition has the following consequence:

12.2. Proposition. Let $Y$ be a cubical subcomplex of some $I(m, l)$ and let $\nu$ denote either the mass or flat metric.

If $\Phi_{1}, \Phi_{2}: Y \rightarrow \mathcal{Z}_{n}(M ; \nu ; G)$ are continuous maps in the mass $\nu$ with

$$
\delta:=\sup \left\{\nu\left(\Phi_{1}(y)-\Phi_{2}(y)\right): y \in Y\right\}<\gamma_{m} \mu_{m}^{-m},
$$

there exists a homotopy $H:[0,1] \times Y \rightarrow \mathcal{Z}_{n}(M ; \nu ; G)$ in the $\nu$ topology between $\Phi_{1}$ and $\Phi_{2}$ such that

$$
\sup \left\{\nu\left(H(y, t)-\Phi_{1}(y)\right):(w, t) \in[0,1] \times Y\right\} \leq \mu_{m}^{m+1} \delta .
$$

Proof. Let $\Psi=\Phi_{2}-\Phi_{1}$ and denote by $Y^{(j)}$ the union of all cells of $Y$ with dimension at most $j$, respectively, for every $j=0, \ldots, m$.

12.3. Claim. For each $j=0, \ldots, m$, there exists a map $H: I \times Y^{(j)} \rightarrow$ $\mathcal{Z}_{n}(M ; \nu ; G)$ that satisfies:

- $H$ is continuous in the $\nu$ topology;

- $H(0, y)=0$ and $H(1, y)=\Psi(y)$ for every $y \in Y^{(j)}$;

- $\sup \left\{\nu(H(w)): w \in[0,1] \times Y^{(j)}\right\} \leq \mu_{m}^{j+1} \delta$.

We will construct the homotopy by an inductive process. Proposition 12.1 gives us the existence of $H: I \times Y^{(0)} \rightarrow \mathcal{Z}_{n}(M ; \nu ; G)$ that satisfies

- $H$ is continuous in the $\nu$ topology;

- $H(0, y)=0$ and $H(1, y)=\Psi(y)$ for every $y \in Y^{(0)}$;

- $\sup \left\{\nu(H(w)): w \in[0,1] \times Y^{(0)}\right\} \leq \mu_{m} \delta$.

Let us suppose now that we have constructed a map $H: I \times Y^{(j-1)} \rightarrow$ $\mathcal{Z}_{n}(M ; \nu ; G)$ that satisfies

- $H$ is continuous in the $\nu$ topology;

- $H(0, y)=0$ and $H(1, y)=\Psi(y)$ for every $y \in Y^{(j-1)}$;

- $\sup \left\{\nu(H(w)): w \in[0,1] \times Y^{(j-1)}\right\} \leq \mu_{m}^{j} \delta$.

We can extend $H$ continuously to $\{1\} \times Y^{(j)}$ by putting $H(1, y)=\Psi(y)$ for each $y \in Y^{(j)}$, and we still have

$$
\sup \left\{\nu(H(w)): w \in\left(I \times Y^{(j-1)}\right) \cup\left(\{1\} \times Y^{(j)}\right)\right\} \leq \mu_{m}^{j} \delta .
$$


Let $\sigma \in Y_{j}^{(j)}$ be a $j$-dimensional cell of $Y$ and choose a homeomorphism $f_{\sigma}: I^{j+1} \rightarrow I \times \sigma$ such that $f_{\sigma}\left(\{1\} \times I^{j}\right)=(\{1\} \times \sigma) \cup(I \times \partial \sigma)$. Then $H \circ f_{\sigma}$ is well-defined on $\{1\} \times I^{j}$. Since $f_{\sigma}\left(\partial\left(\{1\} \times I^{j}\right)\right) \subset\{0\} \times \partial \sigma$, then $\left(H \circ f_{\sigma}\right)(x)=0$ for all $x \in \partial\left(\{1\} \times I^{j}\right)$. Proposition 12.1 gives again a map $H_{\sigma}: I \times I^{j} \rightarrow \mathcal{Z}_{n}(M ; \nu ; G)$ that satisfies:

- $H_{\sigma}$ is continuous in the $\nu$ topology;

- $H_{\sigma}(0, x)=0$ and $H_{\sigma}(1, x)=\left(H \circ f_{\sigma}\right)(x)$ for every $x \in I^{j}$;

- $H_{\sigma}(t, x)=0$ for every $x \in \partial I^{j}$ and $t \in[0,1]$;

- $\sup \left\{\nu\left(H_{\sigma}(w)\right): w \in I \times I^{j}\right\} \leq \mu_{m}^{j+1} \delta$.

We can extend $H$ to a map $H: I \times Y^{(j)} \rightarrow \mathcal{Z}_{n}(M ; \nu ; G)$ by setting $H=H_{\sigma} \circ f_{\sigma}^{-1}$ on each $I \times \sigma, \sigma \in Y_{j}^{(j)}$. This proves the claim.

By applying the claim with $j=m$, we get a homotopy $H$ relative to $Z$ between the zero map and $\Psi=\Phi_{2}-\Phi_{1}$. Then $\tilde{H}(z)=H(z)+\Phi_{1}(z)$ for $z \in Y \times I$ is the desired homotopy.

12.4. Proof of Theorem 2.5. We need the following proposition.

12.5. Proposition. Given $\Pi_{\mathbf{M}}$ in $\left[X, \mathcal{Z}_{n}(M ; \mathbf{M} ; G)\right]$ there is

$$
\Pi \in\left[X, \mathcal{Z}_{n}(M ; \mathbf{M} ; G)\right]^{\#}
$$

so that

(1) for all $\Psi \in \Pi_{\mathbf{M}}$ there is $S=\left\{\psi_{i}\right\}_{i \in \mathbb{N}} \in \Pi$ so that

$$
\mathbf{L}(S)=\sup \{\mathbf{M}(\Psi(x)): x \in X\}
$$

and

$$
\limsup _{i \rightarrow \infty} \max \left\{\mathbf{M}\left(\Psi(x)-\psi_{i}(x)\right): x \in \operatorname{dmn}\left(\psi_{i}\right)\right\}=0 ;
$$

(2) for all $S^{\prime} \in \Pi$ there is a sequence $\left\{\Psi_{j}\right\}_{j \in \mathbb{N}}$ in $\Pi_{\mathbf{M}}$ so that

$$
\mathbf{L}\left(S^{\prime}\right)=\mathbf{L}\left(\left\{\Psi_{j}\right\}_{j \in \mathbb{N}}\right) \text { and } \mathbf{C}\left(S^{\prime}\right)=\mathbf{C}\left(\left\{\Psi_{j}\right\}_{j \in \mathbb{N}}\right) .
$$

In particular, $\mathbf{L}(\Pi)=\mathbf{L}\left(\Pi_{\mathbf{M}}\right)$.

Proof. Choose $\Phi \in \Pi_{\mathbf{M}}$. For each $i \in \mathbb{N}$ pick $k_{i} \in \mathbb{N}$ so that

$$
x, x^{\prime} \in X \quad \text { and } \quad\left|x-x^{\prime}\right|<3^{-k_{i}} \Longrightarrow \mathbf{M}\left(\Phi(x)-\Phi\left(x^{\prime}\right)\right)<1 / i
$$

and set $\phi_{i}: X\left(k_{i}\right)_{0} \rightarrow \mathcal{Z}_{n}(M ; G)$ to be $\phi_{i}(x)=\Phi(x)$. Then $\left\{\phi_{i}\right\}_{i \in \mathbb{N}}$ is a $(X, \mathbf{M})$-homotopy sequences of mappings into $\mathcal{Z}_{n}(M ; \mathbf{M} ; G)$ and we denote its homotopy class by $\Pi$.

We now prove (1). For any $\Psi \in \Pi_{\mathbf{M}}$ there is a homotopy $H$ between $\Phi$ and $\Psi$. Thus, for each $i \in \mathbb{N}$ choose $j_{i} \in \mathbb{N}$ so that

$$
\begin{aligned}
&(s, x),\left(s^{\prime}, x^{\prime}\right) \in[0,1] \times X \text { and } \quad\left|(s, x)-\left(s^{\prime}, x^{\prime}\right)\right|<3^{-j_{i}} \\
& \Longrightarrow \mathbf{M}\left(H(s, x)-H\left(s^{\prime}, x^{\prime}\right)\right)<1 / i .
\end{aligned}
$$


Set

$$
\psi_{i}: X\left(j_{i}\right)_{0} \rightarrow \mathcal{Z}_{n}(M), \quad \psi_{i}(x)=H(1, x) .
$$

Like before, we have that $S=\left\{\psi_{i}\right\}_{i \in \mathbb{N}}$ is a $(X, \mathbf{M})$-homotopy sequences of mappings into $\mathcal{Z}_{n}(M ; \mathbf{M} ; G)$ that belongs to $\Pi$. Moreover, from the construction of $S$ follows at once that

$$
\mathbf{L}(S)=\sup \{\mathbf{M}(\Psi(x)): x \in X\}
$$

and

$$
\limsup _{i \rightarrow \infty} \max \left\{\mathbf{M}\left(\Psi(x)-\psi_{i}(x)\right): x \in \operatorname{dmn}\left(\psi_{i}\right)\right\}=0 .
$$

We now prove (2). Given $S^{\prime}=\left\{\psi_{i}\right\}_{i \in \mathbb{N}} \in \Pi$, let $\sigma_{i}: I\left(1, l_{i}\right)_{0} \times X\left(m, l_{i}\right)_{0} \rightarrow$ $\mathcal{Z}_{n}(M ; G)$ be the $X$-homotopy between $\psi_{i}$ and $\phi_{i}$ with fineness smaller than $\delta_{i}$, where $\delta_{i}$ tends to zero.

Let $H_{i}$ be the Almgren extension given by Theorem 11.16 of $\sigma_{i}$ (which is defined for all $i \geq i_{1}$ for some $i_{1} \in \mathbb{N}$ ) and set

$$
\begin{aligned}
\Psi_{i}: X \rightarrow \mathcal{Z}_{n}(M ; \mathbf{M} ; G) & x \mapsto H_{i}(0, x) \\
\hat{\Phi}_{i}: X \rightarrow \mathcal{Z}_{n}(M ; \mathbf{M} ; G) & x \mapsto H_{i}(1, x) .
\end{aligned}
$$

Theorem 11.16 implies as well that

$$
\mathbf{L}\left(S^{\prime}\right)=\mathbf{L}\left(\left\{\Psi_{i}\right\}_{i \in \mathbb{N}, i \geq i_{1}}\right) \text { and } \mathbf{C}\left(S^{\prime}\right)=\mathbf{C}\left(\left\{\Psi_{i}\right\}_{i \in \mathbb{N}, i \geq i_{1}}\right) .
$$

We need to show that $\Psi_{i} \in \Pi_{\mathbf{M}}$ for all $i$ large. Indeed, from Theorem 11.16 and the way $\phi_{i}$ was constructed, we also have that for all $i \geq i_{1}$

$$
\sup \left\{\mathbf{M}\left(\hat{\Phi}_{i}(x)-\Phi(x)\right): x \in X\right\} \leq\left(C_{0}+1\right) \delta_{i}+1 / i .
$$

Thus Proposition 12.2 implies that, for all $i$ sufficiently large, there is a homotopy in the mass topology between $\hat{\Phi}_{i}$ and $\Phi$. But $\Psi_{i}$ is homotopic in the mass topology to $\Phi_{i}$ and so $\Psi_{i} \in \Pi_{\mathbf{M}}$ for all $i$ large.

We now prove Theorem 2.5. Given $\Pi_{\mathbf{M}}$ in $\left[X, \mathcal{Z}_{n}(M ; \mathbf{M} ; G)\right]$ consider the discrete homotopy class $\Pi$ given by Proposition 12.5 . We have $\mathbf{L}(\Pi)=$ $\mathbf{L}\left(\Pi_{\mathbf{M}}\right)>0$ and thus we can apply Theorem 11.14. Hence there is a smooth minimal hypersurface $\Sigma$ (with multiplicities) so that $\mathbf{L}\left(\Pi_{\mathbf{M}}\right)=\|\Sigma\|(M)$.

If $\left\{\Phi_{i}\right\}_{i \in \mathbb{N}}$ is an optimal sequence, we want to show that $\Sigma$ can be chosen to belong to $\mathbf{C}\left(\left\{\Phi_{i}\right\}_{i \in \mathbb{N}}\right)$. Proposition 12.5 (1) gives us a sequence $S^{i}=$ $\left\{\phi_{j}^{i}\right\}_{j \in \mathbb{N}} \in \Pi$ with

$$
\lim _{i \rightarrow \infty} \mathbf{L}\left(S^{i}\right)=\mathbf{L}(\Pi)
$$

and

$$
\limsup _{j \rightarrow \infty} \max \left\{\mathbf{M}\left(\Phi_{i}(x)-\phi_{j}^{i}(x)\right): x \in \operatorname{dmn}\left(\phi_{j}^{i}\right)\right\}=0 .
$$

Pick an increasing sequence $\left\{n_{i}\right\}_{i \in \mathbb{N}}$ so that we have, for all $j \geq n_{i}$,

- $\max \left\{\mathbf{M}\left(\phi_{j}^{i}(x)\right): x \in \operatorname{dmn}\left(\phi_{j}^{i}\right)\right\} \leq \mathbf{L}\left(S^{i}\right)+1 / i$;

- $\max \left\{\mathbf{M}\left(\Phi_{i}(x)-\phi_{j}^{i}(x)\right): x \in \operatorname{dmn}\left(\phi_{j}^{i}\right)\right\} \leq 1 / i$;

- $\phi_{j}^{i}$ is $X$-homotopic with $\phi_{j+1}^{i}$ with fineness $1 / i$; 
- and $\phi_{j}^{k}$ is $X$-homotopic with $\phi_{j}^{k+1}$ with fineness $1 / i$ for any $1 \leq k \leq$ $i$.

Let $\phi_{j}^{*}$ be given by $\phi_{j}^{*}=\phi_{j}^{1}$ if $j \leq n_{2}-1$, and $\phi_{j}^{*}=\phi_{j}^{i}$ if $n_{i} \leq j \leq n_{i+1}-1$. Then $S^{*}=\left\{\phi_{j}^{*}\right\} \in \Pi$ and $\mathbf{L}\left(S^{*}\right)=\mathbf{L}(\Pi)$, which means $S^{*}$ is a critical sequence. From Theorem 2.5 we know that $\Sigma$ can be chosen so that $\Sigma \in$ $\mathbf{C}\left(S^{*}\right)$ and, from the construction of $S^{*}$, we have $\mathbf{C}\left(S^{*}\right) \subset \mathbf{C}\left(\left\{\Phi_{i}\right\}_{i \in \mathbb{N}}\right)$.

Finally, we want to find an optimal sequence $\left\{\Psi_{i}\right\}_{i \in \mathbb{N}}$ so that every element in $\mathbf{C}\left(\left\{\Psi_{i}\right\}_{i \in \mathbb{N}}\right)$ is a stationary varifold. Theorem 2.5 gives a critical sequence $S$ so that every element in $\mathbf{C}(S)$ is a stationary varifold and thus Proposition 12.5 (2) gives us the desired optimal sequence $\left\{\Psi_{i}\right\}_{i \in \mathbb{N}}$.

12.6. Proof of Theorem 2.9. Given $\Phi: X \rightarrow \mathcal{Z}_{n}(M ; G)$ a continuous map in the flat topology with no concentration of mass, consider $S=\left\{\phi_{i}\right\}_{i \in \mathbb{N}}$ the $(X, \mathbf{M})$-homotopy sequence of mappings into $\mathcal{Z}_{n}(M ; \mathbf{M} ; G)$ given by Theorem 11.18. Let $\sigma_{i}: I\left(1, l_{i}\right)_{0} \times X\left(m, l_{i}\right)_{0} \rightarrow \mathcal{Z}_{n}(M ; G)$ be the $X$-homotopy between $\phi_{i}$ and $\phi_{i+1}$ with fineness smaller than $\delta_{i}$, where $\delta_{i}$ is a sequence tending to zero.

Let $H_{i}$ be the Almgren extension given by Theorem 11.16 of $\sigma_{i}$ (which is defined for all $i \geq i_{1}$ for some $i_{1} \in \mathbb{N}$ ) and set

$$
\begin{gathered}
\Phi_{i}: X \rightarrow \mathcal{Z}_{n}(M ; \mathbf{M} ; G) \quad x \mapsto H_{i}(0, x) \\
\hat{\Phi}_{i+1}: X \rightarrow \mathcal{Z}_{n}(M ; \mathbf{M} ; G) \quad x \mapsto H_{i}(1, x) .
\end{gathered}
$$

Theorem 11.16 implies as well that

$$
\mathbf{L}\left(\left\{\Phi_{i}\right\}_{i \in \mathbb{N}, i \geq i_{1}}\right)=\mathbf{L}(S) \leq \sup \{\mathbf{M}(\Phi(x)): x \in X\}
$$

and note that we also have

$$
\sup \left\{\mathbf{M}\left(\hat{\Phi}_{i+1}(x)-\Phi_{i+i}(x)\right): x \in X\right\} \leq 2\left(C_{0}+1\right) \delta_{i} .
$$

Thus Proposition 12.2 implies that, for all $i$ sufficiently large, there is a homotopy in the mass topology between $\hat{\Phi}_{i+1}$ and $\Phi_{i+1}$. But $\Phi_{i}$ is homotopic in the mass topology to $\hat{\Phi}_{i+1}$ and so all $\Phi_{i}$ belong to the same homotopy class, that we denote by $\Pi_{\mathbf{M}}$.

We now argue that $\Pi_{\mathbf{M}} \subset[\Phi]$. From Theorem 11.16 (i), (iii), and Theorem 11.18 (iii) we have that

$$
\lim _{i \rightarrow \infty} \sup \left\{\mathcal{F}\left(\Phi_{i}(x)-\Phi(x)\right): x \in X\right\}=0
$$

and so Proposition 12.2 implies that for all $i$ sufficiently large $\Phi_{i} \in[\Phi]$, which means that $\Pi_{\mathbf{M}} \subset[\Phi]$. This completes the proof.

12.7. Proof of Theorem 2.12. Let $c_{0}$ be the center of the cube $I^{m}$ and let $Q_{t}$ denote the cube with center $c_{0}$ and side length $t, 0<t \leq 1$. Consider also the homeomorphism

$$
R_{t}: Q_{t} \rightarrow I^{m}, \quad R_{t}(x)=\frac{1}{t}\left(x-c_{0}\right)+c_{0}
$$

and set $t_{i}=1-(2 i)^{-1}, i \in \mathbb{N}$. 
12.8. Proposition. Given $\Pi_{\mathbf{M}}$ in $\pi_{m}\left(\mathcal{Z}_{n}(M ; \mathbf{M}), \Phi_{0}\right)$ there is

$$
\Pi \in \pi_{m}^{\#}\left(\mathcal{Z}_{n}(M ; \mathbf{M}), \Phi_{0}\right)
$$

so that

(1) for all $\Psi \in \Pi_{\mathbf{M}}$ there is $S=\left\{\psi_{i}\right\}_{i \in \mathbb{N}} \in \Pi$ so that

$$
\mathbf{L}(S)=\sup \left\{\mathbf{M}(\Psi(x)): x \in I^{m}\right\}
$$

and

$\limsup _{i \rightarrow \infty} \max \left\{\mathbf{M}\left(\Psi \circ R_{t_{i}}^{-1}(x)-\psi_{i}(x)\right): x \in \operatorname{dmn}\left(\psi_{i}\right)\right\}=0 ;$

(2) for all $S^{\prime} \in \Pi$ there is a sequence $\left\{\Psi_{j}\right\}_{j \in \mathbb{N}}$ in $\Pi_{\mathbf{M}}$ so that

$$
\mathbf{L}\left(S^{\prime}\right)=\mathbf{L}\left(\left\{\Psi_{j}\right\}_{j \in \mathbb{N}}\right) \quad \text { and } \quad \mathbf{C}\left(S^{\prime}\right)=\mathbf{C}\left(\left\{\Psi_{j}\right\}_{j \in \mathbb{N}}\right) .
$$

In particular, $\mathbf{L}(\Pi)=\mathbf{L}\left(\Pi_{\mathbf{M}}\right)$.

Proof. Choose $\Phi \in \Pi_{\mathbf{M}}$. We have a continuous function in the $\mathbf{F}$-metric

$$
F: I^{m} \times(0,1] \rightarrow \mathcal{Z}_{n}(M ; \mathbf{F}), \quad F(x, t)=\Phi \circ R_{t}^{-1}(x)
$$

that is continuous in the mass topology when $F$ is restricted to $I^{m} \times(0,1)$. Thus, for each $i \in \mathbb{N}$ choose $k_{i} \in \mathbb{N}$ so that for every

$$
(x, t),\left(x^{\prime}, t^{\prime}\right) \in I^{m} \times\left[t_{i}, t_{i+1}\right] \text { with }\left|(x, t)-\left(x^{\prime}, t^{\prime}\right)\right|<3^{-k_{i}}
$$

we have that

$$
\mathbf{M}\left(F(x, t)-F\left(x^{\prime}, t^{\prime}\right)\right)<1 / i
$$

Set

$$
\phi_{i}: I\left(m, k_{i}\right)_{0} \rightarrow \mathcal{Z}_{n}(M), \quad \phi_{i}(x)=F\left(x, t_{i}\right) .
$$

Note that, because $F$ is continuous in the $\mathbf{F}$-metric and $F(x, 1)=\Phi_{0}(x)$ for all $x \in \partial I^{m}$, we have that for all $\delta>0$ there is $0<\bar{t}<1$ so that

$$
\mathbf{M}(F(x, t)) \leq M\left(\Phi_{0}(x)\right)+\delta \quad \text { and } \quad \mathcal{F}\left(F(x, t)-\Phi_{0}(x)\right) \leq \delta
$$

for all $(x, t) \in \partial I^{m} \times[\bar{t}, 1]$.

As a result we obtain that $\left\{\phi_{i}\right\}_{i \in \mathbb{N}}$ is a $\left(I^{m}, \mathbf{M}\right)$-homotopy sequence of mappings into $\left(\mathcal{Z}_{n}(M ; \mathbf{M}), \Phi_{0}\right)$ and we denote its homotopy class by $\Pi$.

We now prove (1). For any $\Psi \in \Pi_{\mathbf{M}}$ there is a homotopy $H$ between $\Phi-\Psi$ and zero in the mass topology relative to $\partial I^{m}$. Consider the continuous function in the $\mathbf{F}$-metric

$F_{1}:[0,1] \times I^{m} \times(0,1] \rightarrow \mathcal{Z}_{n}(M ; \mathbf{F}), \quad F_{1}(s, x, t)=H\left(s, R_{t}^{-1}(x)\right)+\Psi \circ R_{t}^{-1}(x)$.

$F_{1}$ is continuous in the mass topology when restricted to $[0,1] \times I^{m} \times(0,1)$. Thus, for each $i \in \mathbb{N}$ choose $j_{i} \in \mathbb{N}$ so that for every $(s, x, t),\left(s^{\prime}, x^{\prime}, t^{\prime}\right) \in[0,1] \times I^{m} \times\left[t_{i}, t_{i+1}\right] \quad$ with $\quad\left|(s, x, t)-\left(s^{\prime}, x^{\prime}, t^{\prime}\right)\right|<3^{-j_{i}}$ we have that

$$
\mathbf{M}\left(F_{1}(s, x, t)-F_{1}\left(s^{\prime}, x^{\prime}, t^{\prime}\right)\right)<1 / i
$$


Set

$$
\psi_{i}: I\left(m, j_{i}\right)_{0} \rightarrow \mathcal{Z}_{n}(M), \quad \psi_{i}(x)=F_{1}\left(1, x, t_{i}\right)=\Psi \circ R_{t_{i}}^{-1}(x) .
$$

Like before, we have that $S=\left\{\psi_{i}\right\}_{i \in \mathbb{N}}$ is a $\left(I^{m}, \mathbf{M}\right)$-homotopy sequences of mappings into $\left(\mathcal{Z}_{n}(M ; \mathbf{M}), \Phi_{0}\right)$ that belongs to $\Pi$. Moreover, from the construction of $S$ follows at once that

$$
\mathbf{L}(S)=\sup \left\{\mathbf{M}(\Psi(x)): x \in I^{m}\right\}
$$

and

$$
\limsup _{i \rightarrow \infty} \max \left\{\mathbf{M}\left(\Psi \circ R_{t_{i}}^{-1}(x)-\psi_{i}(x)\right): x \in \operatorname{dmn}\left(\psi_{i}\right)\right\}=0 .
$$

We now prove (2). For each $0<t^{\prime}<t \leq 1$ consider homeomorphisms $R_{t^{\prime}, t}: \overline{Q_{t} \backslash Q_{t^{\prime}}} \rightarrow[0,1] \times \partial I^{m}$ so that

$$
R_{t^{\prime}, t}(x)=\left(0, R_{t^{\prime}}(x)\right) \quad \text { if } \quad x \in \partial Q_{t^{\prime}}
$$

and

$$
R_{t^{\prime}, t}(x)=\left(1, R_{t}(x)\right) \quad \text { if } \quad x \in \partial Q_{t} .
$$

Given $S^{\prime}=\left\{\psi_{i}\right\}_{i \in \mathbb{N}} \in \Pi$, let $\sigma_{i}: I\left(1, l_{i}\right)_{0} \times I\left(m, l_{i}\right)_{0} \rightarrow \mathcal{Z}_{n}(M)$ be the $m$-homotopy between $\psi_{i}$ and $\phi_{i}$ with fineness smaller than $\delta_{i}$, where $\delta_{i}$ tends to zero.

Let $H_{i}^{1}$ be the Almgren extension given by Theorem 11.16 of $\sigma_{i}$ (which is defined for all $i \geq i_{1}$ for some $i_{1} \in \mathbb{N}$ ) and set

$$
\begin{aligned}
\hat{\Psi}_{i}: I^{m} \rightarrow \mathcal{Z}_{n}(M ; \mathbf{M}) & x \mapsto H_{i}^{1}(0, x) \\
\hat{\Phi}_{i}: I^{m} \rightarrow \mathcal{Z}_{n}(M ; \mathbf{M}) & x \mapsto H_{i}^{1}(1, x) .
\end{aligned}
$$

From Definition 11.6 (iii) we have that for all $i \geq i_{1}$

$$
\begin{aligned}
& \sup \left\{\mathbf{M}\left(H_{i}^{1}(s, x)\right)-\mathbf{M}\left(\Phi_{0}(x)\right):(s, x) \in[0,1] \times \partial I^{m}\right\} \leq\left(C_{0}+1\right) \delta_{i}, \\
& \sup \left\{\mathcal{F}\left(H_{i}^{1}(s, x)-\Phi_{0}(x)\right):(s, x) \in[0,1] \times \partial I^{m}\right\} \leq\left(C_{0}+1\right) \delta_{i} .
\end{aligned}
$$

In particular, the two inequalities above when combined with Lemma 2.2 imply that

$$
\limsup _{i \rightarrow \infty} \sup \left\{\mathbf{F}\left(H_{i}^{1}(s, x), \Phi_{0}(x)\right):(s, x) \in[0,1] \times \partial I^{m}\right\}=0 .
$$

Theorem 11.16 implies as well that

$$
\mathbf{L}\left(S^{\prime}\right)=\mathbf{L}\left(\left\{\hat{\Psi}_{i}\right\}_{i \in \mathbb{N}, i \geq i_{1}}\right) \quad \text { and } \quad \mathbf{C}\left(S^{\prime}\right)=\mathbf{C}\left(\left\{\hat{\Psi}_{i}\right\}_{i \in \mathbb{N}, i \geq i_{1}}\right) .
$$

Set $\Phi_{i}=\Phi \circ R_{t_{i}}^{-1}$. From Theorem 11.16 and the way $\phi_{i}$ was constructed, we have that for all $i \geq i_{1}$

$$
\sup \left\{\mathbf{M}\left(\hat{\Phi}_{i}(x)-\Phi_{i}(x)\right): x \in I^{m}\right\} \leq\left(C_{0}+1\right) \delta_{i}+1 / i .
$$

The inequality above and Proposition 12.2 imply the existence of $i_{2} \in \mathbb{N}$ so that for all $i \geq i_{2}$ we have a homotopy in the mass topology $H_{i}^{2}:[0,1] \times I^{m} \rightarrow$ $\mathcal{Z}_{n}(M ; \mathbf{M})$ between $\hat{\Phi}_{i}$ and $\Phi_{i}$. Moreover

$$
\limsup _{i \rightarrow \infty} \sup \left\{\mathbf{F}\left(H_{i}^{2}(s, x), \Phi_{0}(x)\right):(s, x) \in[0,1] \times \partial I^{m}\right\}=0 .
$$


Consider, for all $i \geq i_{2}$, the function $\Psi_{i}: I^{m} \rightarrow \mathcal{Z}_{n}(M)$ given by

$$
\Psi_{i}(x)=\left\{\begin{aligned}
\hat{\Psi}_{i}\left(R_{t_{i} / 3}(x)\right) & \text { if } x \in Q_{t_{i} / 3}, \\
H_{i}^{1}\left(R_{t_{i} / 3,2 t_{i} / 3}(x)\right) & \text { if } x \in Q_{2 t_{i} / 3} \backslash Q_{t_{i} / 3}, \\
H_{i}^{2}\left(R_{2 t_{i} / 3, t_{i}}(x)\right) & \text { if } x \in Q_{t_{i}} \backslash Q_{2 t_{i} / 3}, \\
\Phi(x) & \text { if } x \in I^{m} \backslash Q_{t_{i}} .
\end{aligned}\right.
$$

The function $\Phi_{i}$ is continuous in the $\mathbf{F}$-topology and continuous in the mass topology on $I^{m} \backslash \partial I^{m}$. Moreover, $\Psi_{i \mid Q_{t_{i}}}$ is homotopic in the mass topology to $\Phi_{i} \circ R_{t_{i}}=\Phi$ relative to $\partial Q_{t_{i}}$. Thus $\Psi_{i}-\Phi$ is homotopic to zero in the mass topology relative to $\partial I^{m}$ and so $\Psi_{i} \in \Pi_{\mathbf{M}}$ for all $i \geq i_{2}$.

From (23) and (25) we have

$$
\begin{aligned}
\limsup _{i \rightarrow \infty}\left(\sup _{x \in I^{m} \backslash Q_{t_{i} / 3}}\right. & \inf \left\{\mathbf{F}\left(\Psi_{i}(x), \Phi_{0}(y)\right): y \in \partial I^{m}\right\} \\
& \left.+\sup _{y \in \partial I^{m}} \inf \left\{\mathbf{F}\left(\Psi_{i}(x), \Phi_{0}(y)\right): x \in I^{m} \backslash Q_{t_{i} / 3}\right\}\right)=0
\end{aligned}
$$

and hence, recalling (24) and Lemma 11.8, we obtain that

$$
\mathbf{L}\left(\left\{\Psi_{i}\right\}_{i \in \mathbb{N}, i \geq i_{2}}\right)=\mathbf{L}\left(S^{\prime}\right) \text { and } \mathbf{C}\left(\left\{\Psi_{i}\right\}_{i \in \mathbb{N}, i \geq i_{1}}\right)=\mathbf{C}\left(S^{\prime}\right) .
$$

Theorem 2.12 follows by combining Theorem 11.14 with Proposition 12.8 in the same way that Theorem 2.5 followed by combining Theorem 11.14 with Proposition 12.5.

12.9. Proof of Theorem 2.13. Given $\Phi \in \mathcal{E}\left(\Phi_{0}\right)$, consider $S=\left\{\phi_{i}\right\}_{i \in \mathbb{N}}$ the $\left(I^{m}, \mathbf{M}\right)$-homotopy sequence of mappings into $\mathcal{Z}_{n}\left(M ; \mathbf{M} ; \Phi_{0}\right)$ given by Theorem 11.18.

We will use the following notation introduced in Proposition 12.8: $Q_{t}$, $R_{t}, R_{t^{\prime}, t}$, and $t_{i}=1-1 / 2 i$.

Let $\sigma_{i}: I\left(1, l_{i}\right)_{0} \times I\left(m, l_{i}\right)_{0} \rightarrow \mathcal{Z}_{n}(M)$ be the $m$-homotopy between $\phi_{i}$ and $\phi_{i+1}$ with fineness smaller than $\delta_{i}$, where $\delta_{i}$ tends to zero.

Let $H_{i}^{1}$ be the Almgren extension given by Theorem 11.16 of $\sigma_{i}$ (which is defined for all $i \geq i_{1}$ for some $i_{1} \in \mathbb{N}$ ) and set

$$
\begin{aligned}
& \Psi_{i}: I^{m} \rightarrow \mathcal{Z}_{n}(M ; \mathbf{M}) \quad x \mapsto H_{i}^{1}(0, x) \\
& \hat{\Psi}_{i+1}: I^{m} \rightarrow \mathcal{Z}_{n}(M ; \mathbf{M}) \quad x \mapsto H_{i}^{1}(1, x) .
\end{aligned}
$$

Theorem 11.16 implies as well that

$$
\mathbf{L}\left(\left\{\Psi_{i}\right\}_{i \in \mathbb{N}, i \geq i_{1}}\right)=\mathbf{L}(S) \leq \sup \left\{\mathbf{M}(\Phi(x)): x \in I^{m}\right\},
$$

and

$$
\begin{aligned}
& \sup \left\{\mathbf{M}\left(H_{i}^{1}(s, x)\right)-\mathbf{M}\left(\Phi_{0}(x)\right):(s, x) \in[0,1] \times \partial I^{m}\right\} \leq\left(C_{0}+1\right) \delta_{i} \\
& \sup \left\{\mathcal{F}\left(H_{i}^{1}(s, x)-\Phi_{0}(x)\right):(s, x) \in[0,1] \times \partial I^{m}\right\} \leq\left(C_{0}+1\right) \delta_{i}
\end{aligned}
$$


The two inequalities above when combined with Lemma 2.2 imply that

$$
\limsup _{i \rightarrow \infty} \sup \left\{\mathbf{F}\left(H_{i}^{1}(s, x), \Phi_{0}(x)\right):(s, x) \in[0,1] \times \partial I^{m}\right\}=0 .
$$

Moreover from Theorem 11.16 (i), (iii), and Theorem 11.18 (iii) we have that

$$
\lim _{i \rightarrow \infty} \sup \left\{\mathcal{F}\left(\Psi_{i}(x)-\Phi(x)\right): x \in I^{m}\right\}=0 .
$$

Finally, we note that

$$
\sup \left\{\mathbf{M}\left(\hat{\Psi}_{i+1}(x)-\Psi_{i+i}(x)\right): x \in X\right\} \leq 2\left(C_{0}+1\right) \delta_{i}
$$

Proposition $12.2,(26)$, and (28) imply the existence of $i_{2} \geq i_{1} \in \mathbb{N}$ so that for all $i \geq i_{2}$ we have a homotopy in the mass topology $H_{i}^{2}:[0,1] \times I^{m} \rightarrow$ $\mathcal{Z}_{n}(M ; \mathbf{M})$ between $\hat{\Psi}_{i+1}$ and $\Psi_{i+1}$ such that

$$
\limsup _{i \rightarrow \infty} \sup \left\{\mathbf{F}\left(H_{i}^{2}(s, x), \Phi_{0}(x)\right):(s, x) \in[0,1] \times \partial I^{m}\right\}=0 .
$$

Thus, combining $H_{i}^{1}$ with $H_{i}^{2}$, we obtain a homotopy between $\Psi_{i}$ and $\Psi_{i+1}$ given by $H_{i}:[0,1] \times I^{m} \rightarrow \mathcal{Z}_{n}(M ; \mathbf{M})$, where

$$
\limsup _{i \rightarrow \infty} \sup \left\{\mathbf{F}\left(H_{i}(s, x), \Phi_{0}(x)\right):(s, x) \in[0,1] \times \partial I^{m}\right\}=0 .
$$

Consider, for all $i \geq i_{2}$, the function $\Phi_{i}: I^{m} \rightarrow \mathcal{Z}_{n}(M)$ given by

$$
\Phi_{i}(x)=\left\{\begin{aligned}
\Psi_{i} \circ R_{t_{i}}(x) & \text { if } x \in Q_{t_{i}} \\
H_{j}\left(R_{t_{j+1}, t_{j}}(x)\right) & \text { if } x \in Q_{t_{j+1}} \backslash Q_{t_{j}} \text { and } j \geq i \\
\Phi_{0}(x) & \text { if } x \in \partial I^{m}
\end{aligned}\right.
$$

The function $\Phi_{i}$ is continuous in the mass topology on $I^{m} \backslash \partial I^{m}$ and, due to (29), is also continuous in the $\mathbf{F}$-topology on $I^{m}$. Thus $\Phi_{i} \in \mathcal{E}\left(\Phi_{0} ; \mathbf{M}\right)$ for all $i \geq i_{2}$. Note that (29) implies as well that

$$
\mathbf{L}\left(\left\{\Phi_{i}\right\}_{i \in \mathbb{N}, i \geq i_{2}}\right)=\mathbf{L}\left(\left\{\Psi_{i}\right\}_{i \in \mathbb{N}, i \geq i_{1}}\right)=\mathbf{L}(S) .
$$

We remark that $\Phi_{i}=\Phi_{i+1}$ on $I^{m} \backslash \overline{Q_{t_{i+1}}}$. Moreover, $\Phi_{i \mid Q_{t_{i+1}}}$ is homotopic in the mass topology to $\Phi_{i+1 \mid Q_{t_{i+1}}}$ relative to $\partial Q_{t_{i+1}}$. Thus $\Phi_{i+1}-\Phi_{i}$ is homotopic to zero in the mass topology relative to $\partial I^{m}$ and so $\Phi_{i}$ belong all to the same homotopy class $\Pi_{\mathbf{M}}$.

To finish the proof we need to show that $\Pi_{\mathbf{M}} \subset[\Phi]$. Consider the auxiliary maps $\Omega_{i}: I^{m} \rightarrow \mathcal{Z}_{n}(M)$ given by

$\Omega_{i}(x)=\Phi \circ R_{t_{i}}(x) \quad$ if $x \in Q_{t_{i}} \quad$ and $\quad \Omega_{i}(x)=\hat{\Phi}_{0} \circ R_{1, t_{i}}(x) \quad$ if $x \in I^{m} \backslash Q_{t_{i}}$, where $\hat{\Phi}_{0}:[0,1] \times \partial I^{m} \rightarrow \mathcal{Z}_{n}(M)$ is given by $\hat{\Phi}_{0}(t, x)=\Phi_{0}(x)$. The map $\Omega_{i}$ belongs to $\mathcal{E}\left(\Phi_{0}\right)$ and to $[\Phi]$. From $(27)$ we have

$$
\lim _{i \rightarrow \infty} \sup \left\{\mathcal{F}\left(\Psi_{i} \circ R_{t_{i}}(x)-\Phi \circ R_{t_{i}}(x)\right): x \in Q_{t_{i}}\right\}=0
$$


and hence we obtain from (29) that

$$
\lim _{i \rightarrow \infty} \sup \left\{\mathcal{F}\left(\Phi_{i}(x)-\Omega_{i}(x)\right): x \in I^{m}\right\}=0 .
$$

Thus we obtain from Proposition 12.1 that $\Phi_{i}-\Omega_{i}$ is homotopic to zero in the flat topology relative to $\partial I^{m}$ for all $i$ large, which means that $\Phi_{i} \in[\Phi]$ for all $i \geq i_{2}$.

\section{References}

[1] I. Agol, F. C. Marques and A. Neves, Min-max theory and the energy of links arXiv:1205.0825 [math.GT] (2012) 1-19.

[2] F. Almgren, The homotopy groups of the integral cycle groups, Topology (1962), 257299.

[3] F. Almgren, The theory of varifolds. Mimeographed notes, Princeton (1965).

[4] F. Almgren, Some interior regularity theorems for minimal surfaces and an extension of Bernstein's theorem. Ann. of Math. (2) 84 (1966), 277-292.

[5] W. Ballmann, Der Satz von Lusternik und Schnirelmann, (German) Beiträge zur Differentialgeometrie, Heft 1, pp. 1-25, Bonner Math. Schriften, 102, Univ. Bonn, Bonn, 1978.

[6] V. Bangert, On the existence of closed geodesics on two-spheres, Internat. J. Math. 4 (1993), 1-10.

[7] G. D. Birkhoff, Dynamical systems with two degrees of freedom, Trans. Amer. Math. Soc. 18 (1917), 199-300.

[8] W. Blaschke, Vorlesungen Über Differentialgeometrie III, Berlin: Springer (1929).

[9] V. Buchstaber and T. Panov, Torus actions and their applications in topology and combinatorics, University Lecture Series, 24. American Mathematical Society, Providence, RI, 2002.

[10] S.S Chern, M. do Carmo, and S. Kobayashi,. Minimal submanifolds of a sphere with second fundamental form of constant length, Functional Analysis and Related Fields, Springer 1970, 59-75.

[11] T. Colding and C. De Lellis, The min-max construction of minimal surfaces, Surveys in Differential Geometry VIII , International Press, (2003), 75-107.

[12] H. Federer, Geometric measure theory, Die Grundlehren der mathematischen Wissenschaften, Band 153 Springer-Verlag New York Inc., New York 1969.

[13] T. Frankel, On the fundamental group of a compact minimal submanifold, Ann. of Math. 83 (1966), 68-73.

[14] J. Franks, Geodesics on $S^{2}$ and periodic points of annulus homeomorphisms, Invent. Math. 108 (1992), 403-418.

[15] M. Freedman, Z-X. He, Z. Wang, Möbius energy of knots and unknots, Ann. of Math. (2) 139 (1994), 1-50.

[16] M. Grayson, Shortening embedded curves, Ann. Math. 120 (1989) 71-112.

[17] M. Gromov, Dimension, nonlinear spectra and width,. Geometric aspects of functional analysis,(1986/87), Lecture Notes in Math., 1317, Springer, Berlin, 1988, 132-184.

[18] M. Gromov, Isoperimetry of waists and concentration of maps, Geom. Funct. Anal. 13 (2003), 178-215.

[19] M. Gromov, Singularities, expanders and topology of maps. I. Homology versus volume in the spaces of cycles. Geom. Funct. Anal. 19 (2009), 743-841.

[20] L. Guth, Minimax problems related to cup powers and Steenrod squares, Geom. Funct. Anal. 18 (2009), 1917-1987.

[21] A. Hatcher, Algebraic Topology, Cambridge University Press (2002)

[22] Zheng-Xu He, On the minimizers of the Möbius cross energy of links, Experiment. Math. 11 (2002), 244-248.

[23] N. Hingston, On the growth of the number of closed geodesics on the two-sphere, Internat. Math. Res. Notices (1993) 253-262. 
[24] D. Kim and R. Kusner, Torus knots extremizing the Möbius energy, Experiment. Math. Volume 2, Issue 1 (1993), 1-9.

[25] W. Klingenberg, Lectures on closed geodesics, Grundlehren der Mathematischen Wissenschaften, Vol. 230. Springer-Verlag, Berlin-New York, 1978.

[26] B. Lawson, Complete minimal surfaces in $S^{3}$, Ann. of Math. (2) 92 (1970), 335-374.

[27] P. Li and S-T. Yau, A new conformal invariant and its applications to the Willmore conjecture and the first eigenvalue of compact surfaces, Invent. Math. 69 (1982), 269291.

[28] L. Lusternik, Topology of functional spaces and calculus of variations in the large, Trav. Inst. Math. Stekloff 19, (1947).

[29] L. Lusternik and L. Schnirelmann, Topological methods in variational problems and their application to the differential geometry of surfaces Uspehi Matem. Nauk (N.S.) 2, (1947), 166-217.

[30] F. C. Marques and A. Neves, Rigidity of min-max minimal spheres in three-manifolds, Duke Math. J. 161 (2012), 2725-2752.

[31] F. C. Marques and A. Neves, Min-max theory and the Willmore conjecture, Ann. of Math. (2) 179 (2014), 683-782.

[32] F. C. Marques and A. Neves Existence of infinitely many minimal hypersurfaces in positive Ricci curvature, arXiv:1311.6501 [math.DG] (2014), 1-33.

[33] F. C. Marques and A. Neves The Willmore conjecture, arXiv:1409.7664 [math.DG] (2014), 1-22.

[34] S. Montiel and A. Ros, Minimal immersions of surfaces by the first eigenfunctions and conformal area, Invent. Math. 83 (1985), 153-166.

[35] F. Morgan, Geometric measure theory. A beginner's guide. Fourth edition. Elsevier/Academic Press, Amsterdam, 2009.

[36] J. O'Hara, Energy of a knot, Topology 30 (1991), 241-247.

[37] J. Pitts, Existence and regularity of minimal surfaces on Riemannian manifolds, Mathematical Notes 27, Princeton University Press, Princeton, (1981).

[38] H. Poincaré, Sur les lignes géodésiques des surfaces convexes, Trans. Amer. Math. Soc. 6 (1905), 237-274.

[39] A. Ros, The Willmore conjecture in the real projective space, Math. Res. Lett. 6 (1999), 487-493.

[40] R. Schoen and L. Simon, Regularity of stable minimal hypersurfaces. Comm. Pure Appl. Math. 34 (1981), 741-797.

[41] L. Simon, Lectures on geometric measure theory, Proceedings of the Centre for Mathematical Analysis, Australian National University, Canberra, (1983).

[42] I. Taimanov, On the existence of three nonintersecting closed geodesics on manifolds that are homeomorphic to the two-dimensional sphere, (Russian) Izv. Ross. Akad. Nauk Ser. Mat. 56 (1992), 605-635.

[43] G. Thomsen, Über Konforme Geometrie, I: Grundlagen der Konformen Flächentheorie, Abh. Math. Sem. Hamburg (1923), 31-56.

[44] F. Urbano, Minimal surfaces with low index in the three-dimensional sphere, Proc. Amer. Math. Soc. 108 (1990), 989-992.

[45] T. J. Willmore, Note on embedded surfaces, An. Sti. Univ. "Al. I. Cuza" Iasi Sect. I a Mat. (N.S.) 11B (1965) 493-496.

[46] S.-T. Yau Problem section. Seminar on Differential Geometry, pp. 669-706, Ann. of Math. Stud., 102, Princeton Univ. Press, Princeton, N.J., 1982.

Instituto de Matemática Pura e Aplicada (IMPA), Estrada Dona CastoRiNA 110, 22460-320 Rio DE JANEIRo, BRAZIL

E-mail address: coda@impa.br

Imperial College, Huxley Building, 180 Queen's Gate, London SW7 2RH, UNITED KINGDOM

E-mail address: a.neves@imperial.ac.uk 